Memoirs of the College of Science, University of Kyoto, Series A Vol. XXXIII, Mathematics No. 3, 1961.

\title{
On the construction of two-dimensional diffusion processes satisfying Wentzell's boundary conditions and its application to boundary value problems
}

By

\author{
Nobuyuki IKEDA
}

(Received Jan. 15, 1961)

0. Introduction. Consider a Markov process on a regular domain $\bar{D}$ in $R^{2}$ whose generator is given as an elliptic differential operator

$$
\begin{array}{r}
A u(x, y)=A_{11}(x, y) \frac{\partial^{2}}{\partial x^{2}} u(x, y)+2 A_{12}(x, y) \frac{\partial^{2}}{\partial x \partial y} u(x, y) \\
+A_{22}(x, y) \frac{\partial^{2}}{\partial y^{2}} u(x, y)+B_{1}(x, y) \frac{\partial}{\partial x} u(x, y)+B_{2}(x, y) \frac{\partial}{\partial y} u(x, y), \\
\quad \text { for } u(x, y) \in C^{2},
\end{array}
$$

in $D$ associated with the boundary condition

$$
\begin{array}{r}
\delta(x, y) \lim _{(x, y, y) \rightarrow(x, y)} A u(x, y)=\frac{\partial}{\partial \xi_{(x, y)}^{2}} u(x, y)+M(x, y) \frac{\partial}{\partial \xi_{(x, y)}^{1}} u(x, y)+V(x, y) \\
\times \frac{\partial^{2}}{\partial \xi_{(x, y)}^{1} \partial_{\zeta(x, y)}^{\xi 1}} u(x, y)+\int_{\partial D}\left\{u\left(x+x^{\prime}, y+y^{\prime}\right)-u(x, y)-\frac{\partial}{\partial \xi_{(x, y)}^{1}} u(x, y)\right. \\
\left.\quad \times \xi_{(x, y)}^{1}\left(x^{\prime}, y^{\prime}\right)\right\} \nu_{(x, y)}\left(d x^{\prime} d y^{\prime}\right), \text { for } u(x, y) \in C^{2},(x, y) \in \partial D,
\end{array}
$$

where $\delta(x, y) \equiv 0$ or $1, V(x, y)$ is non-negative and $\nu_{(x, y)}(\cdot)$ is a measure on $\partial D$ satisfying $\nu_{(x, y)}\left(\partial D-U_{(x, y)}\right)<+\infty$ and $\int_{\partial D}\left(\xi_{(x, y)}^{1}\left(x^{\prime}, y^{\prime}\right)\right)^{2}$ $\nu_{(x, y)}\left(d x^{\prime} d y^{\prime}\right)<+\infty$ for any neighbourhood $U_{(x, y)}$ of $(x, y)$. $\left\{\xi_{(x, y)}^{i}\left(x^{\prime}, y^{\prime}\right), i=1,2\right\}$ is a $C^{2}$-function on $\bar{D}$ and is a local coordinate in $U_{(x, y)}$ satisfying: $\xi_{(x, y)}^{2}\left(x^{\prime} y^{\prime}\right)=0$ if and only if $\left(x^{\prime}, y^{\prime}\right) \in \partial D$. for $\left(x^{\prime}, y^{\prime}\right) \in U_{(x, y)}, \xi_{(x, y)}^{2}\left(x^{\prime}, y^{\prime}\right)+\left(\xi_{(x, y)}^{1}\left(x^{\prime}, y^{\prime}\right)\right)^{2}>0$ if and only if $\left(x^{\prime}, y^{\prime}\right) \neq(x, y)$. It is easily seen from A. D. Wentzell's results [24] that this boundary condition is of the most general type provided 
that the process does not jump from the boundary of $D$ into its interior.

The main purpose of this paper is to construct the path functions of such Markov processes by means of the method of stochastic integral equations initiated by K. Ito [12]. It should be noted that we used not only the ordinal time but also the local time at the boundary in formulation our stochastic integral equation, to meet the difficulty arising from the boundary condition which did not appear in Ito's case.

It suffices for this purpose to discuss the case in which $D$ is a unit disk and $A$ and the boundary condition are given in the polar coordinate as

$$
\begin{array}{r}
A u(r, \theta)=\frac{1}{2}\left\{\frac{\partial^{2}}{\partial r^{2}}+\frac{1}{r} \frac{\partial}{\partial r}+b^{2}(r, \theta) \frac{\partial^{0}}{\partial \theta^{2}}+2 a(r, \theta) \frac{\partial}{\partial \theta}\right\} u(r, \theta) \\
\begin{aligned}
\delta(\theta) \lim _{r \uparrow 1} A u(r, \theta)=\frac{\partial}{\partial n} u(1, \theta)+M(\theta) \frac{\partial}{\partial \theta} u(1, \theta) \\
+(1 / 2) V(\theta) \frac{\partial^{2}}{\partial \theta^{2}} u(1, \theta)+\int_{-\pi}^{\pi}(u(1, \theta+\xi)-u(1, \theta) \\
\left.-\xi \frac{\partial}{\partial \theta} u(1, \theta)\right) n(d \xi ; \theta),
\end{aligned}
\end{array}
$$

where $\delta(\theta) \equiv 0$ or $1, V(\theta)-b^{2}(1, \theta) \delta(\theta)$ is non-negative and $n(\cdot ; \theta)$ is a measure on $\partial D$ satisfying $n\left(\partial D-U_{(\theta)}\right)<+\infty, \int_{\partial D} \xi^{2} n\left(d_{\xi} ; \theta\right)<+\infty$ for any neighbourhood $U_{(\theta)}$ of $(1, \theta)$ and some regularity conditions, for the general case can be reduced to this special one under the some regularity conditions.

Consider two Brownian motions $B(t, \omega)$ and $g(t, \omega)$, Poisson measure $p(d s d u, \omega)$ and the Bessel process $r(t, \omega)$ with the sticky barrier at 1 which are all independent of each other, and denote with $\sigma_{r_{0}}(\omega)$ and $\mathrm{t}(s, \omega)$ the first passage time and the local time at 1 for $r(s, \omega)$ respectively. As is seen in Section 2 the $r(t, \omega)$ proess together with the solution of the stochastic integral equation $\eta(t, \omega)=\int_{0}^{t \wedge \sigma r_{0}(\omega)} a(r(s, \omega), \eta(s, \omega)) d s+\int_{0}^{t \wedge \sigma r_{0}(\omega)} b(r(s, \omega), \eta(s, \omega)) d B(s, \omega)$ 
$+\int_{0}^{\mathrm{t}\left(t \wedge \sigma r_{0}(\omega), \omega\right)} m\left(\eta\left(\mathrm{t}^{-1}(s, \omega), \omega\right)\right) d s+\int_{0}^{\mathrm{t}\left(t \wedge \sigma r_{0}(\omega), \omega\right)} \sigma\left(\eta\left(\mathrm{t}^{-1}(s, \omega), \omega\right)\right) d g(s, \omega)$

$$
\begin{aligned}
& +\int_{0}^{\mathrm{t}\left(t \wedge \sigma_{r_{0}}(\omega), \omega\right)} \int_{1 \leq|u|<+\infty} \beta\left(\xi, \eta\left(\mathrm{t}^{-1}(s, \omega), \omega\right)\right) p(d s d \xi, \omega) \\
& +\int_{0}^{\mathrm{t}\left(t \wedge \sigma_{r_{0}}(\omega), \omega\right)} \int_{|u|<1} \beta\left(\xi, \eta\left(\mathrm{t}^{-1}(s, \omega), \omega\right)\right) q(d s d \xi, \omega),
\end{aligned}
$$

will give the polar coordinates of the Markov process on $D_{r_{0}}$ with the generator described in $(0.3)$ and $(0.4)(\delta(\theta) \equiv 1)$ where $D_{r_{0}}$ $=\left\{(r, \theta) ; 0<r_{0} \leq r \leq 1\right\}, q(B ; \omega)=p(B, \omega)-E(p(B, \omega)), m(\theta)=M(\theta)-$ $a(1, \theta), \sigma^{2}(\theta)=V(\theta)-b^{2}(1, \theta), \beta(\xi, \theta)$ is a non-decreasing and right continuous function in $\xi,-\infty<\xi<+\infty$, with some regularity conditions satisfying : $\beta(u, \pi)=\beta(u,-\pi), \beta(+\infty, \theta)=-\pi, \beta(+\infty, \theta)$ $=\pi, \int_{|\boldsymbol{u}|<+\infty} \beta(\xi, \theta)^{2} d \xi / \xi^{2}<+\infty$ and

$$
\beta(\xi, \theta)= \begin{cases}\inf \left\{a ; n((a, \pi] ; \theta)<\frac{1}{\xi}\right\}, & \text { for } \xi \geqslant 0, \\ \sup \left\{a ; n([-\pi, a) ; \theta)<\frac{-1}{\xi}\right\}, & \text { for } \xi<0 .\end{cases}
$$

We shall need slight modifications to discuss the case “ $\delta(\theta) \equiv 0$ " as is seen in Section 4 .

Our paper consist of two parts.

Part 1 is for the construction of the path functions of our Markov processes. In Section 1, we shall introduce preliminary notions and assumptions which will be used later. Sections 2 and 3 are devoted to the construction of the path functions. Further in these section we shall prove some theorems concerning the strongly Markovian property of the diffusion on a unit disk $\bar{D}$ determined by the stochastic integral equation mentioned above. In Section 4 we shall define a Markov process on the boundary concerning the diffusion on $\bar{D}$ constructed in Section 2 (or Section 3) and prove two theorems (Theorem 4.1 and 4.2) which will play important roles later. In Section 5 we shall give the description of the boundary condition corresponding to the diffusion process on $\bar{D}$ constructed above. We can construct the rotation invariant diffusion without appealing to the stochastic integral equation, as 
is shown in Section 6. In Section 7 we shall give a transform of scale to reduce the general elliptic differential operator to the special one discussed in Sections 3 and 4.

In Part 2 we shall discuss the boundary value problem by using the special case of the process constructed above. Although it is well known that J. L. Doob constructed the solution of the first boundary value problem (Dirichlet problem) probabilistically using the Brownian motion, no analogous method has not yet been known for the second boundary value problem (Neumann problem). We shall give a probabilistic method in Sections 8 and 9 to solve the boundary value problem which is even more general than the second boundary value problem using the Brownian motion with some appropriate boundary condition. Our method will suggest that the second boundary value problem can be discussed probabilistically in the case of the general elliptic differential operator constructed in Part 1 just as the first boundary value problem was extended by several authors such as J. L. Doob [7], and $\mathrm{K}$. Ito [13].

The author is heavily indebted to Professors K. Ito and H.P. McKean for generous help during the work on this problem. Messrs. T. Ueno, H. Tanaka and K. Sato took much interest in the reserach and joined in discussions orally or by communication. The author expresses his thanks to these friends. Thanks are due to Professor T. Hida for his kind and useful opinions on the manuscript.

\section{Part 1. Construction of path functions.}

1. Notations and Assumptions ${ }^{1)}$. Let $S$ be a subset of the $N$-dimensional Euclidean space $R^{N}$. For convenience we add an extra point $\{\infty\}$ to $S$ as an isolated one and get a topological space $S^{*}=S \bigvee\{\infty\}$. For a measurable function $w$ from $[0,+\infty]$ to $S^{*}$, we define

1) This general set up, except the special assumptions, are due to the lectures given by Prof. K. Ito and Prof. H. P. McKean at Kyoto in 1957-8. 


$$
\sigma_{(\infty)}(w)= \begin{cases}\inf \{t ; w(t)=\{\infty\}, t \geq 0\}, & \text { if such } t \text { exists, } \\ +\infty & , \text { if otherwise. }\end{cases}
$$

Let $W$ be the set of all $w$ 's satisfying

(W.1) $\quad w(t)=\infty$

, for $t \geq \sigma_{(\infty)}(w)$,

(W.2) $w(t)$ is right cotinuous and has left limit at for every $t, 0 \leq t<\sigma_{(\infty)}(w)$,

(W. 3) $w(+\infty)=\infty$.

We call $W$ the space of path functions. The value of $w$ at time $t$ is denoted by $w(t), x(t, w), \eta(t, w), l(t, w)$ and so on (or simply $w_{t}, x_{t}, \eta_{t}, l_{t}$ and so on). Let $\mathcal{F}(S)$ be the family of all Borel susets of $S, \mathscr{B}$ the Borel field generated by the $w$-sets of the form $\{w ; w(s) \in B\}, s \geq 0, B \in \mathscr{F}(S)$, and $\mathscr{B}_{t}$ the Borel field generated by the $w$-sets of the form $\{w ; w(s) \in B\}, 0 \leq s \leq t$, $B \in \mathcal{F}(S)$. Given $\sigma(w)$ a non-negative function measurable $(\mathscr{B})$, we shall define the stopped (shifted) path $w_{\sigma}^{-}\left(w_{\sigma}^{+}\right)$:

$$
\left(w_{\sigma}^{-}\right)_{t}= \begin{cases}w_{t}, & t<\sigma, \\ w_{\sigma}, & \sigma \leq t<+\infty, \quad\left(w_{\sigma}^{+}\right)_{t}=w_{t+\sigma}, \\ \infty, & t=+\infty,\end{cases}
$$

and the associated Borel fields:

$\mathscr{B}_{\sigma}=$ the Borel field generated by the $w$-sets of the form $\left\{w ; w_{\sigma}^{-} \in B\right\}, B \in \mathscr{B}$,

$\mathscr{B}_{\sigma+}=\bigcap_{n_{21}} \mathscr{B}_{\sigma+1 / n}$.

We assume that a family of probability measure $P_{x}(\cdot), x \in S$, over $\mathscr{B}$ is given and satisfies the following conditions:

(P.1) For any fixed $B \in \mathscr{B}, \mathrm{P}_{x}(B)$ is Borel measurable in $x$.

$$
P_{x}(x(0, w)=x)=1, \quad x \in S .
$$

(P.3) (Markov property)

$$
P_{x}\left\{P_{x}\left(w_{t}^{+} \in B / \mathscr{B}_{t}\right)=P_{x_{t}}(w \in B)\right\}=1, \quad B \in \mathscr{B} .
$$

$\mathfrak{M}=\left\{W, x(t, w), \mathscr{B}, P_{x}, x \in S\right\}$ is called a Markov process on 
$S$ with right continuous paths, and it is often denoted simply by $\{x(t, w), t \geq 0\}$.

We shall introduce function spaces $\mathscr{B}(K), \mathcal{C}(K)$ and $\mathcal{C}^{n}(K)$. $\mathscr{B}(K)$ is the space of all bounded measurable functions defined on $K^{*}$ taking the value 0 at $\{\infty\} . \mathcal{C}(K)$ is the space of all bounded functions defined on $K^{*}$, continuous with respect to the relative topology and taking the value 0 at $\{\infty\} . C^{n}(K)$ is the space of all $n$-times continuously differentiable functions on $K$ and vanishing at $\{\infty\}$. The norm in $\mathscr{B}(K)$ as well as in $\mathcal{C}(K)$ is given by $\|f\|=\sup _{x \in K}|f(x)|$.

As is usual theory of Markov processes we shall define the transition probabilities, the semi-group and Green operators associated with our processes. We write

$$
\begin{aligned}
& P(t, x, E)=P_{x}(x(t, w) \in E), \text { for } E \in \mathscr{F}(S), \text { (transition probability), } \\
& T_{t} f(x)=E_{x}\{f(x(t, w))\}, f \in \mathscr{B}(S),(\text { semi-group }), \\
& G_{a} f(x)=E_{x}\left\{\int_{0}^{+\infty} e^{-\alpha t} f(x(t, w)) d t\right\}, \quad f \in \mathscr{B}(S), \alpha>0, \\
& \text { (Green operator). }
\end{aligned}
$$

The hitting time $\sigma_{E}$ for a set $E \in \mathscr{F}(S)$ is defined by

$$
\sigma_{E}(w)= \begin{cases}\inf \{t ; x(t, w) \in E, t \geq 0\}, & \text { if such } t \text { exists, } \\ +\infty & , \text { if otherwise. }\end{cases}
$$

A random time $\sigma(w)$ is called a Markov time, if

$$
\{w ; \sigma(w)<t\} \in \mathscr{B}_{t}, \text { for } 0 \leq t<+\infty .
$$

A Markov process $\mathfrak{M}$ on $S$ with right continuous paths is called a strong Markov process, if for any Markov time $\sigma(w)$,

$$
P_{x}\left\{P\left(w_{\sigma}^{+} \in B / \mathscr{B}_{\sigma+}\right)=P_{x(\sigma(w), w)}(w \in B)\right\}=1, \quad x \in S \text { and } B \in \mathscr{B} .
$$

A strong Markov process $\mathfrak{M}$ on $S$ with right continuous paths is called a diffusion process, if any path is continuous as far as it runs in the interior of $S^{2}$.

2) This definition is somewhat different from ordinary one (Cf. K. Ito and H. P. McKean [14] and E. B. Dynkin [6]). 
Let $D$ be a unit circular disk with closure $\bar{D}$ and the boundary $\partial D$, that is, $D=\left\{x ; x=(r, \theta) \in R^{2}, 0 \leq r<1\right\}$. We use the abbreviation of $f(\theta)$ instead of $f(1, \theta) \in \mathcal{C}(\partial D)$.

Throught this paper, we shall denote with $A$

$$
A u(x)=A u(r, \theta)=\frac{1}{2}\left\{\frac{\partial^{2}}{\partial r^{2}}+\frac{1}{r} \frac{\partial}{\partial r}+b^{2}(r, \theta) \frac{\partial^{2}}{\partial \theta^{2}}+2 a(r, \theta) \frac{\partial}{\partial \theta}\right\} u(r, \theta),
$$

where $a(r, \theta)$ and $b(r, \theta)$ satisfy some regularity conditions, for example, $a(r, \theta), b(r, \theta) \in C^{2}(S)$, and the domain $\mathscr{D}(A)$ of $A$ is a certain subset of $\mathcal{C}^{2}(S)$.

The reflecting barrier Bessel process is defined as a diffusion process $\mathfrak{M}=\left\{W, r(t, w), \mathcal{B}, P_{r}, r \in[0,1]\right\}$ with Feller's invariants

$$
m(d r)=2 r d r \quad \text { and } \quad s(d r)=r^{-1} d r
$$

and the boundary condition

$$
\frac{d^{-}}{d r} u(1)=0^{3},
$$

and the sticky barrier Bessel process is defined by replacing the boundary condition above with

$$
\lim _{r \uparrow 1} \frac{d}{d m} \frac{d}{d s} u(r)=\frac{d^{-}}{d r} u(1) .
$$

The infinitely divisible law of probability whose logarithmic characteristic function (l.c. f.) is

$$
\psi_{0}(z)=i z-z^{2} / 2+\int_{|u|>1}\left(e^{i u z}-1\right) d u / u^{2}+\int_{|u| \leq \mid}\left(e^{i u z}-1-i z u\right) d u / u^{2},
$$

will be called the fundamental infinitely divisible law (i. d. 1.) in this paper. A Markov process with $S=(-\infty,+\infty)$ is said to be differential if its probability law is translation invariant. A differential Markov process $\mathfrak{M}=\left\{W, l(t, w), \mathcal{B}, P_{r}, r \in R^{1}\right\}$ on $R^{1}$ is called a fundamental Lévy process, if the probability law of $l(s, w)-$ $l(t, w)$ has the 1.c. f. $(s-t) \psi_{0}(z)$, where $\psi_{0}(z)$ is the 1.c. f. of the fundamental i.d. l.. Any jump of $l(t, w)$ is expressed by a point

3) $\frac{d^{-}}{d r} u$ means the left derivative of $u$. 
$(t, u) \in[0,+\infty) \times R^{1}, t$ being its position and $u$ being its hight: $l(t, w)-l(t-0, w)$. The number $p(E, w)$ of the jumps in $E, E \in$ $\mathscr{F}\left([0,+\infty) \times R^{1}\right)$, can be considered a real random variable, which proves to be governed by the Poisson distribution with the mean

$$
\pi(E)=\int_{E} d \tau d u / u^{2}
$$

$p(E, w)$ is evidently a function of $l(t, w) . \quad l(t, w), 0 \leq t<+\infty$, can be expressed as

$l(t, w)-l(0, w)=t+g(t, w)+\int_{0}^{t} \int_{|u|>\mid} u p(d \tau d u, w)+\int_{0}^{t} \int_{|u|<1} u q(d \tau d u, w)$, for any $t, 0 \leq t<+\infty$, for almost all $w$, where $q(E, w)=p(E, w)-$ $\pi(E)$ and $\{g(t, w), 0 \leq t<+\infty\}$ is a Brownian motion, which is also a function of $\{l(t, w), 0 \leq t<+\infty\}$, (Cf. P. Lévy [17] or K. Ito $[10])$.

2. Construction of path functions (1). In this section, we shall construct the diffusion in the unit disk whose generator and boundary condition are given by $(0.3)$ and $(0.4)$ with " $\delta(\theta) \equiv 1$ ". To do this, we use the stochastic integral equation.

Let $a(r, \theta)$ and $b(r, \theta)$ be the real valued functions ${ }^{4)}$, defined on $\bar{D}$ satisfying the following conditions:

$$
a(r, \theta), b(r, \theta) \in \mathcal{C}(\bar{D}-0) .
$$

(A. 2) For any $(r, \theta),\left(r^{\prime}, \theta^{\prime}\right) \in \bar{D}_{r_{0}}$,

and

$$
\left|a(r, \theta)-a\left(r^{\prime}, \theta^{\prime}\right)\right| \leq A\left\{\left|\theta-\theta^{\prime}\right|+\left|r-r^{\prime}\right|\right\},
$$

$$
\left|b(r, \theta)-b\left(r^{\prime}, \theta^{\prime}\right)\right| \leq B\left\{\left|\theta-\theta^{\prime}\right|+\left|r-r^{\prime}\right|\right\},
$$

where $r_{0}$ is any fixed positive number $<1$ and $\bar{D}_{r_{0}}$ is the annulus $\left\{x=(r, \theta): r_{0} \leq r \leq 1\right\}$, and $A=A_{r_{0}}$ and $B=B_{r_{0}}$ are independent of $r$ and $\theta$, but may depend on $r_{0}$ in general.

(A. 3) For any $x=(r, \theta) \in \bar{D}$,

$$
b(r, \theta)>0 .
$$

4) It is automatic from this that $a(r, \theta)$ and $b(r, \theta)$ are periodic in $\theta$ with the period $2 \pi$, and this remark is available to all functions defined on $\bar{D}$. 
Let $m(1, \theta)(\equiv m(\theta))$ and $\sigma(1, \theta)(\equiv \sigma(\theta))$ be the real valued functions, defined on $\partial D$, satisfying the following conditions:

$$
m(\theta), \sigma(\theta) \in C(\partial D) \text {. }
$$

(B. 2$)$ For any $(1, \theta),\left(1, \theta^{\prime}\right) \in \partial D$,

$$
\left|m(\theta)-m\left(\theta^{\prime}\right)\right| \leq M\left|\theta-\theta^{\prime}\right| \text { and }\left|\sigma(\theta)-\sigma\left(\theta^{\prime}\right)\right| \leq S\left|\theta-\theta^{\prime}\right| .
$$

(B. 3) For any $(1, \theta) \in \partial D$,

$$
\sigma(\theta) \geqq 0
$$

Let $\beta(u, \theta)$ be the real valued function, defined on $(-\infty,+\infty)$ $\times(-\infty,+\infty)$ satisfying the following conditions;

(C. 1) For any $u \in(-\infty,+\infty)$

$$
\beta(u, \theta)=\beta(u, \theta+2 n \pi), \quad n=0, \pm 1, \pm 2, \cdots,
$$

and for any $\theta \in(-\infty,+\infty) \beta(u, \theta)$ is non-decreasing in $u$ and

$$
\beta(-\infty, \theta)=-\pi \text { and } \beta(+\infty, \theta)=\pi .
$$

(C. 2) For any $\theta, \theta^{\prime} \in(-\infty,+\infty)$,

$$
\left\|\left|\beta(u, \theta)-\beta\left(u, \theta^{\prime}\right)\right|\right\| \equiv\left\{\int_{|u|<+\infty}\left|\beta(u, \theta)-\beta\left(u, \theta^{\prime}\right)\right| d u / u^{2}\right\}^{1 / 2} \leq F\left|\theta-\theta^{\prime}\right|,
$$

where $F$ is independent of $\theta$ and $\theta^{\prime}$.

(C. 3) For any $\theta \in(-\infty,+\infty), \beta(u, \theta)$ is a right continuous function in $u$ and

$$
\int_{|u|<+\infty} \beta(u, \theta)^{2} d u / u^{2}<+\infty
$$

Let $\left\{W^{(1)}, r\left(t, w^{(1)}\right), \mathscr{B}^{(1)}, P_{r}^{(1)}, r \in[0,1]\right\}$ be a sticky barrier Bessel process, $\left\{W^{(2)}, B\left(t, w^{(2)}\right), \mathscr{B}^{(2)}, P_{r}^{(2)}, r \in R^{1}\right\}$ a one-dimensional Brownian motion and $\left\{W^{(3)}, l\left(t, w^{(3)}\right), \mathscr{B}^{(3)}, P_{r}^{(3)}, r \in R^{1}\right\}$ a fundamental Lévy process. Denote an arbitrarily fixed random variable on a probability space $\left(\Omega^{(4)}, \mathscr{B}^{(4)}, P^{(4)}\right)$ by $C\left(\omega^{(4)}\right)$ where $\mathscr{B}^{(4)}$ is a Borel field generated by subsets of $\Omega^{(4)}$.

Now we can form a product probability space $\left\{\Omega, \mathscr{B}, P_{r}\right.$, $r \in[0,1]\}$, where $\Omega=W^{(1)} \times W^{(2)} \times W^{(3)} \times \Omega^{(4)}, \quad \mathscr{B}=\mathscr{B}^{(1)} \times \mathcal{B}^{(2)} \times \mathcal{B}^{(3)}$ $\times \mathscr{B}^{(4)}$ and $P_{r}=P_{r}^{(1)} \times P_{0}^{(2)} \times P_{0}^{(3)} \times P^{(4)}$ in the usual way. 
We define a random variable $\mathfrak{t}\left(t, w^{(1)}\right)$ by

$$
\mathrm{t}\left(t, w^{(1)}\right)=\int_{0}^{t} x_{(1)}\left(r\left(s, w^{(1)}\right)\right) d s^{5)} .
$$

Following K. Ito and H. P. McKean [14], we can prove that

$$
P_{1}^{(1)}\left\{\mathrm{t}\left(t, w^{(1)}\right)>0\right\}=1, \text { for any } t>0 .
$$

Let $\mathrm{t}^{-1}\left(t, w^{(1)}\right)$ be the inverse function of $\mathrm{t}\left(t, w^{(1)}\right)$, i. e., for any $t \geqq 0$

$$
\mathrm{t}^{-1}\left(t, w^{(1)}\right)=\inf \left\{s ; \mathrm{t}\left(s, w^{(1)}\right)>t\right\} .
$$

We shall consider a stochastic integral equation, for any $\tau \geq 0$,

$$
\begin{aligned}
& \eta(t, \omega)=C\left(\omega^{(s)}\right)+\int_{\tau \wedge \sigma_{r 0}\left(w^{(1)}\right)}^{t \wedge \sigma_{r_{0}(w(1))}} a\left(r\left(s, w^{(1)}\right), \eta(s, \omega)\right) d s \\
& +\int_{\tau \wedge \sigma_{r_{0}\left(w^{(1)}\right)}}^{t \wedge \sigma_{r_{0}}(\omega(1))} b\left(r\left(s, w^{(1)}\right), \eta(s, \omega)\right) d B\left(s, w^{(2)}\right) \\
& +\int_{\mathrm{t}\left(\tau \wedge \sigma_{r 0}\left(w^{(1)}\right), w^{(1)}\right)}^{\mathrm{t}\left(t \wedge \sigma_{r_{0}}(w(1)), w(1)\right)} m\left(\eta\left(\mathrm{t}^{-1}\left(s, w^{(1)}\right), \omega\right)\right) d s \\
& +\int_{\mathrm{t}\left(\tau \wedge \sigma_{r 0}\left(w^{(1)}\right), w^{(1)}\right)}^{\mathrm{t}\left(t \wedge \sigma_{r_{0}}\left(w(1), w^{(1)}\right)\right.} \sigma\left(\eta\left(\mathrm{t}^{-1}\left(s, w^{(1)}\right), \omega\right)\right) d g\left(s, w^{(3)}\right) \\
& +\int_{\mathrm{t}\left(\tau \wedge \sigma_{r_{0}}\left(w^{(1)}\right), w^{(1)}\right)}^{\mathrm{t}\left(t \wedge \sigma_{r^{(}}(w(1)), w^{(1)}\right)} \int_{|\leq| u \mid<+_{\infty}} \beta\left(u, \eta\left(\mathrm{t}^{-1}\left(s, w^{(1)}\right), \omega\right)\right) p\left(d s d u, w^{(3)}\right) \\
& +\int_{\mathrm{t}\left(t \wedge \sigma_{r_{0}}\left(w^{(1)}\right), w^{(1)}\right)}^{\mathrm{t}\left(t \wedge \sigma_{r_{0}}(w(1)), w^{(1)}\right)} \int_{|u| \leq \mid} \beta\left(u, \eta\left(\mathrm{t}^{-1}\left(s, w^{(1)}\right), \omega\right)\right) q\left(d s d u, \omega^{(3)}\right)^{6)},
\end{aligned}
$$

where $1>r_{0}>0$.

First using the methods similar to those in K. Ito [12], we shall prove the following.

LEMMA 2.1. Let $r_{0}$ be an any positive number such that $0<r_{0}<1$. Then, for any fixed $w^{(1)}$ (up to $P^{(1)}-$ measure 0$)$, there exists one and only one (up to $P$-measure $O$ ) stochastic process $\eta(t, \omega)$ satisfying the stochastic integral equation (2.1) for any $t, \tau \leq t<+\infty$, and fulfilling the following property:

$(\eta): \eta(t, \omega), s \leq t<+\infty$, is a Borel measurable function of

5) $\chi_{F}(*)$ means the indicator function of a set $E$.

6) $a \wedge b$ means the smaller of $a$ and $b$. 
$\eta(s, \omega), r\left(t^{\prime}, w_{s}^{(1)+}\right), l\left(\mathrm{t}\left(t^{\prime}, w_{s}^{(1)+}\right), w_{\mathrm{t}\left(s, w^{(1)}\right)}^{(3)+}\right)-l\left(0, w_{\mathrm{t}\left(s, w^{(1)}\right)}^{(3)+}\right)$ and $B\left(t^{\prime}, w_{s}^{(2)+}\right)$ $-B\left(0, w_{s}^{(2)+}\right), 0 \leq t^{\prime} \leq t-s$.

Proof. We note that the $P_{r}^{(1)}$-measure of the $w^{(1)}$-set

$$
W_{r_{0}}^{(1)}=\left\{w^{(1)} ; \sigma_{r_{0}}\left(w^{(1)}\right)<+\infty\right\}
$$

is 1 , for any $r, r_{0} \leq r \leq 1$.

It is therefore enought to prove that Lemma 2.1 holds for any $w^{(1)} \in W_{r_{0}}^{(1)}$.

We introduce a probability space $\{\widetilde{\Omega}, \tilde{\mathcal{B}}, \widetilde{P}\}$, where $\widetilde{\Omega}=W^{(2)} \times$ $W^{(3)} \times \Omega^{(4)}, \tilde{\mathscr{B}}=\mathscr{B}^{(2)} \times \mathscr{B}^{(3)} \times \mathscr{B}^{(4)}$ and $\tilde{P}=P_{0}^{(2)} \times P_{0}^{(3)} \times P^{(4)}$.

Firstly we shall prove the existence and uniqueness of the stochastic integral equation (2.1) in the case $\left|C\left(\omega^{(4)}\right)\right| \leq N$, i. e., $C_{N}\left(\omega^{(4)}\right)=x_{[-N, N]}\left(C\left(\omega^{(4)}\right)\right) C\left(\omega^{(4)}\right)$.

In order to find a solution we make use of the method of successive approximations ; we define $\eta_{n}(t, \omega), n=0,1,2, \cdots$, recursively by

and

$$
\eta_{0}(t, \omega)=C_{N}\left(\omega^{(4)}\right),
$$

$$
\begin{aligned}
& \eta_{n}(t, \omega)=C_{N}+\int_{\tau \wedge \sigma_{r 0}}^{t \wedge \sigma_{r_{0}}} a\left(r(s), \eta_{n-1}(s, \omega)\right) d s \\
& +\int_{\tau \wedge \sigma_{r 0}}^{t \wedge \sigma_{r_{0}}} b\left(r(s), \eta_{n^{-1}}(s, \omega)\right) d B(s)+\int_{\mathrm{t}\left(\tau \wedge \sigma_{0}\right)}^{\mathrm{t}\left(t \wedge \sigma_{r_{0}}\right)} m\left(\eta_{n^{-1}}\left(\mathrm{t}^{-1}(s), \omega\right)\right) d s \\
& +\int_{\mathrm{t}\left(\tau \wedge \sigma_{\left.r_{0}\right)}\right)}^{\mathrm{t}\left(\mathrm{t} \wedge \sigma_{r_{0}}\right)} \sigma\left(\eta_{n^{-1}}\left(\mathrm{t}^{-1}(s), \omega\right)\right) d g(s) \\
& +\int_{\mathrm{t}\left(\tau \wedge \sigma \sigma_{0}\right)}^{\mathrm{t} t\left(\wedge \sigma_{0}\right)} \int_{1 \leq|u|<+\infty} \beta\left(u, \eta_{n-1}\left(\mathrm{t}^{-1}(s), \omega\right)\right) p(d s d u) \\
& +\int_{\mathrm{t}\left(\tau \wedge \sigma_{r_{0}}\right)}^{\mathrm{t}\left(t \wedge \sigma r_{r^{\prime}}\right)} \int_{|u|<1} \beta\left(u, \eta_{n^{-1}}\left(\mathrm{t}^{-1}(s), \omega\right)\right) q(d s d u) .
\end{aligned}
$$

By (2.2), $n=1$, we have, for any $t, \tau \leq t \leq \sigma_{r_{0}}$,

$$
\begin{aligned}
& \widetilde{E}\left\{\left[\eta_{1}(t, \omega)\right]^{2}\right\} \\
& \leq \\
& \quad\left[\widetilde{E}\left\{\left(C_{N}\right)^{2}\right\}+\widetilde{E}\left\{\left(\int_{\tau}^{t} a\left(r(s), C_{N}\right) d s\right)^{2}\right\}\right. \\
& \quad+\widetilde{E}\left\{\left(\int_{\tau}^{t} b\left(r(s), C_{N}\right) d B(s)\right)^{2}\right\}+\widetilde{E}\left\{\left(\int_{\mathrm{t}(\tau)}^{\mathrm{t}(t)} m\left(C_{N}\right) d s\right)^{2}\right\} \\
& \quad+\widetilde{E}\left\{\left(\int_{\mathrm{t}(\tau)}^{\mathrm{t}(t)} \sigma\left(C_{N}\right) d g(s)\right)^{2}\right\}+\widetilde{E}\left\{\left(\int_{\mathrm{t}(\tau)}^{\mathrm{t}(t)} \int_{|\leq| u \mid<+\infty} \beta\left(u, C_{N}\right) d u d s / u^{2}\right)^{2}\right\} \\
& \left.\quad+\widetilde{E}\left\{\left(\int_{\mathrm{t}(\tau)}^{\mathrm{t}(t)} \int_{|u|<+\infty} \beta\left(u, C_{N}\right) q(d s d u)\right)^{2}\right\}\right],
\end{aligned}
$$


and write this as

$$
\begin{aligned}
& 7\left[\widetilde{E}\left\{I_{1,0}\right)^{2}\right\}+\widetilde{E}\left\{\left(I_{1,1}\right)^{2}\right\}+\widetilde{E}\left\{\left(I_{1,2}\right)^{2}\right\}+\widetilde{E}\left\{\left(I_{1,3}\right)^{2}\right\} \\
& \left.\quad+\widetilde{E}\left\{\left(I_{1,4}\right)^{2}\right\}+\widetilde{E}\left\{\left(I_{1,5}\right)^{2}\right\}+\widetilde{E}\left\{\left(I_{1,6}\right)^{2}\right\}\right] .
\end{aligned}
$$

It is easily seen that for any $t, \tau \leq t \leq \sigma_{r_{0}}$,

$$
\begin{aligned}
& \widetilde{E}\left\{\left(I_{1,0}\right)^{2}\right\} \leq N^{2}, \quad \widetilde{E}\left\{\left(I_{1,1}\right)^{2}\right\} \leq\left(\bar{A}_{r_{0}} \sigma_{r_{0}}\right)^{2}, \\
& \widetilde{E}\left\{\left(I_{1,2}\right)^{2}\right\}=\int_{\tau}^{t} \widetilde{E}\left\{\left(b\left(r(s), C_{N}\right)\right)^{2}\right\} d s \leq\left(\bar{B}_{r_{0}}\right)^{2} \sigma_{r_{0}}, \\
& \widetilde{E}\left\{\left(I_{1,3}\right)^{2}\right\} \leq\left(\bar{M} \sigma_{r_{0}}\right)^{2}, \quad \widetilde{E}\left\{\left(I_{1,4}\right)^{2}\right\} \leq(\bar{S})^{2} \sigma_{r_{0}}, \\
& \widetilde{E}\left\{\left(I_{1,5}\right)^{2}\right\} \leq 2 \sigma_{r_{0}} \int_{t(\tau)}^{t(t)} \widetilde{E}\left\{\left.\left\|\beta\left(u, C_{N}\right)\right\|\right|^{2}\right\} d s \leq 2\left(\sigma_{r_{0}} \bar{F}\right)^{2}, \\
& \widetilde{E}\left\{\left(I_{1,6}\right)^{2}\right\} \leq \int_{t(\tau)}^{t(t)} \widetilde{E}\left\{\left.\left\|\beta\left(u, C_{N}\right)\right\|\right|^{2}\right\} d s \leq \sigma_{r_{0}} \bar{F}^{2},
\end{aligned}
$$

where $\bar{A}_{r_{0}}=\max _{(r, \theta) \in \bar{D} r_{0}}|a(r, \theta)|, \bar{B}_{r_{0}}=\max _{(r, \theta) \in \overline{\bar{D}} r_{0}} b(r, \theta), \bar{M}=\max _{(1, \theta) \in \partial D}|m(\theta)|$, $\bar{S}=\max _{(1, \theta) \in \partial D} \sigma(\theta)$, and $\bar{F}=\max _{(1, \theta) \in \partial D}\|\beta(u, \theta)\| \|$. In the estimation above, we used the fact $\mathrm{t}\left(t, w^{(1)}\right) \leq t$, for any $t, 0 \leq t<+\infty$.

Thus $\widetilde{E}\left\{\left(\eta_{1}(t, \omega)\right)^{2}\right\}, \tau \leq t<+\infty$, is bounded and also $\widetilde{E}\left\{\mid \eta_{1}(t, \omega)\right.$ $\left.-\left.\eta_{0}(t, \omega)\right|^{2}\right\}, \tau \leq t<+\infty$, is bounded. Furthermore $\eta_{1}(t, \omega)$ belongs to $d_{1}$-class with $\widetilde{P}$-measure 1 and so measurable in $(t, \omega)^{7)}$. Besides, for any fixed $w^{(1)} \in W_{r_{0}}^{(1)},\left\{\eta_{1}(t, \omega), l\left(\mathrm{t}\left(s, w_{\tau}^{(1)+}\right), w_{\mathrm{t}\left(\tau, w^{(1)}\right)}^{(3)+}\right)-l\left(0, w_{\mathrm{t}\left(\tau, w^{(1)}\right)}^{(3)+}\right)\right.$, $\left.B\left(s, w_{\tau}^{(2)+}\right)-B\left(0, w_{\tau}^{(2)+}\right), \quad 0 \leq s \leq t-\tau\right\}, \quad$ is $\quad$ independent of $\left\{l\left(\mathrm{t}\left(s, w_{t}^{(1)+}\right), w_{\mathrm{t}\left(t, w^{(1)}\right)}^{(3)+}-l\left(0, w_{\mathrm{t}\left(t, w^{(1)}\right)}^{(3)+}\right), B\left(s, w_{t}^{(2)+}\right)-B\left(0, w_{t}^{(2)+}\right), \quad t \leq s\right.\right.$ $\leq \sigma_{r_{0}}\left(w^{(1)}\right)-t$, for any $\tau \leq t \leq \sigma_{r_{0}}\left(w^{(1)}\right)$, as is easily verified.

Thus we can define $\eta_{2}(t, \omega)$ by $(2.2)$ and so $\eta_{n}(t, \omega), n=1,2, \cdots$, recursively and we have for any $t, \tau \leq t \leq \sigma_{r_{0}}$,

$$
\begin{aligned}
& \eta_{n+1}(t, \omega)-\eta_{n}(t, \omega) \\
& =\int_{\tau}^{t}\left\{a\left(r(s), \eta_{n}(s, \omega)\right)-a\left(r(s), \eta_{n-1}(s, \omega)\right)\right\} d s \\
& \quad+\int_{\tau}^{t}\left\{b\left(r(s), \eta_{n}(s, \omega)\right)-b\left(r(s), \eta_{n-1}(s, \omega)\right)\right\} d B(s) \\
& \quad+\int_{\mathrm{t}(\tau)}^{\mathrm{t}(t)}\left\{m\left(\eta_{n}\left(\mathrm{t}^{-1}(s), \omega\right)\right)-m\left(\eta_{n^{-1}}\left(\mathrm{t}^{-1}(s), \omega\right)\right)\right\} d s
\end{aligned}
$$

7) We say that $f(t)$ belongs to $d_{1}$-class if $f(t)$ is continuous in $t$ except for discontinuities of the first kind. 


$$
\begin{aligned}
& +\int_{\mathrm{t}(\tau)}^{\mathrm{t}(t)}\left\{\sigma\left(\eta_{n}\left(\mathrm{t}^{-1}(s), \omega\right)\right)-\sigma\left(\eta_{n-1}\left(\mathrm{t}^{-1}(s), \omega\right)\right)\right\} d g(s) \\
& +\int_{\mathrm{t}(\tau)}^{\mathrm{t}(t)} \int_{|\leq| u \mid<+\infty}\left\{\beta\left(u, \eta_{n}\left(\mathrm{t}^{-1}(s), \omega\right)\right)-\beta\left(u, \eta_{n-1}\left(\mathrm{t}^{-1}(s), \omega\right)\right)\right\} d u d s / u^{2} \\
& +\int_{\mathrm{t}(\tau)}^{\mathrm{t}(t)} \int_{|u|<+\infty}\left\{\beta\left(u, \eta_{n}\left(\mathrm{t}^{-1}(s), \omega\right)\right)-\beta\left(u, \eta_{n-1}\left(\mathrm{t}^{-1}(s), \omega\right)\right)\right\} q(d s d u, \omega),
\end{aligned}
$$

and write this as

$$
I_{2,1}+I_{2,2}+I_{2,3}+I_{2,4}+I_{2,5}+I_{2,6} \text {. }
$$

Using (A.2), (B.2) and (C.2) we have for any $t, \tau \leq t \leq \sigma_{r_{0}}$,

$$
\begin{aligned}
& \widetilde{E}\left\{\left(I_{2,1}\right)^{2}\right\} \leq A_{r_{0}}^{2} \sigma_{r_{0}} \int_{\tau}^{t} \widetilde{E}\left\{\left|\eta_{n}(s, \omega)-\eta_{n-1}(s, \omega)\right|^{2}\right\} d s, \\
& \widetilde{E}\left\{\left(I_{2,2}\right)^{2}\right\} \leq B_{r_{0}}^{2} \int_{\tau}^{t} \widetilde{E}\left\{\left|\eta_{n}(s, \omega)-\eta_{n-1}(s, \omega)\right|^{2}\right\} d s, \\
& \widetilde{E}\left\{\left(I_{2,3}\right)^{2}\right\} \leq M^{2} \sigma_{r_{0}} \widetilde{E}\left\{\int_{\mathrm{t}(\tau)}^{\mathrm{t}(t)}\left|\eta_{n}\left(\mathrm{t}^{-1}(s), \omega\right)-\eta_{n-1}\left(\mathrm{t}^{-1}(s), \omega\right)\right|^{2} d s\right\} \\
& \quad \leq M^{2} \sigma_{r_{0}} \widetilde{E}\left\{\int_{\tau}^{t}\left|\eta_{n}(s, \omega)-\eta_{n-1}(s, \omega)\right|^{2} \mathrm{t}(d s)\right\} \\
& \quad \leq M^{2} \sigma_{r_{0}} \int_{\tau}^{t} \widetilde{E}\left\{\left|\eta_{n}(s, \omega)-\eta_{n-1}(s, \omega)\right|^{2}\right\} d s,
\end{aligned}
$$

(2.4) $\hat{E}\left\{\left(I_{2,4}\right)^{2}\right\} \leq S^{2} \int_{\mathrm{t}(\tau)}^{\mathrm{t}(t)} \widetilde{E}\left\{\left|\eta_{n}\left(\mathrm{t}^{-1}(s), \omega\right)-\eta_{n-1}\left(\mathrm{t}^{-1}(s), \omega\right)\right|^{2}\right\} d s$

$$
\begin{aligned}
& \leq S^{2} \int_{\tau}^{t} \widetilde{E}\left\{\left|\eta_{n}(s, \omega)-\eta_{n-1}(s, \omega)\right|^{2}\right\} d s, \\
& \widetilde{E}\left\{\left(I_{2,5}\right)^{2}\right\} \leq 2 F^{2} \sigma_{r_{0}} \widetilde{E}\left\{\int_{\mathrm{t}(\tau)}^{\mathrm{t}(t)}\left|\eta_{n}\left(\mathrm{t}^{-1}(s), \omega\right)-\eta_{n-1}\left(\mathrm{t}^{-1}(s), \omega\right)\right|^{2} d s\right\} \\
& \leq 2 F^{2} \sigma_{r_{0}} \int_{\tau}^{t} \widetilde{E}\left\{\left|\eta_{n}(s, \omega)-\eta_{n-1}(s, \omega)\right|^{2}\right\} d s, \\
& \widetilde{E}\left\{\left(I_{2,6}\right)^{2}\right\} \leq F^{2} \widetilde{E}\left\{\int_{\mathrm{t}(\tau)}^{\mathrm{t}(t)}\left|\eta_{n}\left(\mathrm{t}^{-1}(s), \omega\right)-\eta_{n-1}\left(\mathrm{t}^{-1}(s), \omega\right)\right|^{2} d s\right\} \\
& \leq F^{2} \int_{\tau}^{t} \widetilde{E}\left\{\left|\eta_{n}(s, \omega)-\eta_{n-1}(s, \omega)\right|^{2}\right\} d s .
\end{aligned}
$$

Combining (2.3) and (2.4) we have for any $t, \tau \leq t<+\infty$,

$$
\begin{aligned}
\widetilde{E}\left\{\left|\eta_{n+1}(t, \omega)-\eta_{n}(t, \omega)\right|^{2}\right\} \\
\leq K_{1} \int_{\tau \wedge \sigma_{r 0}}^{t \wedge \sigma_{r_{0}}} \widetilde{E}\left\{\left|\eta_{n}(s, \omega)-\eta_{n-1}(s, \omega)\right|^{2}\right\} d s,
\end{aligned}
$$

where $K_{1}=6\left[A_{r_{0}}^{2} \sigma_{r_{0}}+B_{r_{0}}^{2}+M^{2} \sigma_{r_{0}}+S^{2}+2 F^{2} \sigma_{r_{0}}+F^{2}\right]$. 
But $\widetilde{E}\left\{\left|\eta_{1}(t, \omega)-\eta_{0}(t, \omega)\right|^{2}\right\}, \tau \leq t<+\infty$, has a finite upper bound $G$, as is proved above. We obtain recursively for any $t, \tau \leq t \leq \sigma_{r 0}$

$$
\begin{aligned}
& \widetilde{E}\left\{\left|\eta_{n+1}(t, \omega)-\eta_{n}(t, \omega)\right|^{2}\right\} \leq K_{1}^{n-1} G t^{n-1} / n !, \\
& \widetilde{E}\left\{\left[\int_{\tau}^{t}\left(a\left(r(s), \eta_{n}(s, \omega)\right)-a\left(r(s), \eta_{n-1}(s, \omega)\right)\right) d s\right]^{2}\right\} \\
& \leq K_{1}^{n-1} G A_{r_{0}}^{2} \sigma_{r_{0}} t^{n} / n !, \\
& \widetilde{E}\left\{\left[\int_{\tau}^{t}\left(b\left(r(s), \eta_{n}(s, \omega)\right)-b\left(r(s), \eta_{n-1}(s, \omega)\right)\right) d B(s)\right]^{2}\right\} \\
& \leq K_{1}^{n-1} G B_{r_{0}}^{2} t^{n} / n !, \\
& \widetilde{E}\left\{\left[\int_{\mathrm{t}(\tau)}^{\mathrm{t}(t)}\left(m\left(\eta_{n}\left(\mathrm{t}^{-1}(s), \omega\right)\right)-m\left(\eta_{n-1}\left(\mathrm{t}^{-1}(s), \omega\right)\right)\right) d s\right]^{2}\right\} \\
& \leq K_{1}^{n-1} G M^{2} \sigma_{r_{0}} t^{n} / n !, \\
& \widetilde{E}\left\{\left[\int_{\mathrm{t}(\tau)}^{\mathrm{t}(t)}\left(\sigma\left(\eta_{n}\left(\mathrm{t}^{-1}(s), \omega\right)\right)-\sigma\left(\eta_{n-1}\left(\mathrm{t}^{-1}(s), \omega\right)\right)\right) d g(s)\right]^{2}\right\} \\
& \leq K_{1}^{n-1} G S^{2} t^{n} / n !, \\
& \widetilde{E}\left\{\left[\int _ { \mathrm { t } ( \tau ) } ^ { \mathrm { t } ( t ) } \int _ { 1 \leq | u | < + \infty } \left(\beta\left(u, \eta_{n}\left(\mathrm{t}^{-1}(s), \omega\right)\right)\right.\right.\right. \\
& \leq 2 K_{1}^{n-1} G F^{2} \sigma_{r_{0}} t^{n} / n !, \\
& \widetilde{E}\left\{\left[\int _ { \mathrm { t } ( \tau ) } ^ { \mathrm { t } ( t ) } \int _ { | u | < + \infty } \left(\beta\left(u, \eta_{n}\left(\mathrm{t}^{-1}(s), \omega\right)\right)\right.\right.\right. \\
& \\
& \leq K_{1}^{n-1} G F^{2} t^{n} / n ! .
\end{aligned}
$$

Now putting $t=\sigma_{r_{0}}\left(w^{(1)}\right)$ in (2.6) and using Tschebycheff's inequality, we obtain for any fixed $w^{(1)} \in W_{r_{0}}^{(1)}$,

$$
\begin{aligned}
& \tilde{P}\left\{\operatorname{Sup}_{\tau \leq t \leq \sigma r_{0}}\left|\int_{\tau}^{t}\left[a\left(r(s), \eta_{n}(s, \omega)\right)-a\left(r(s), \eta_{n-1}(s, \omega)\right)\right] d s\right| \geq \lambda_{n}{ }^{1 / 4}\right\} \\
& \quad \leq \tilde{P}\left\{\int_{\tau}^{\sigma r_{0}}\left|a\left(r(s), \eta_{n}(s, \omega)\right)-a\left(r(s), \eta_{n-1}(s, \omega)\right)\right| d s \geq \lambda_{n}{ }^{1 / 4}\right\} \\
& \leq \lambda_{n}{ }^{1 / 2},
\end{aligned}
$$

where $\lambda_{n}=K_{1}^{n-1} G A_{r_{0}}^{2}\left(\sigma_{r_{0}}\right)^{n+1} / n$ !. Since $\sum_{n=1}^{+\infty} \lambda_{n}{ }^{1 / 4}, \sum_{n=1}^{+\infty} \lambda_{n}{ }^{1 / 2}<+\infty$, for any fixed $w^{(1)} \in W_{r_{0}}^{(1)}$, 
(2.7)

$$
\begin{array}{r}
\sum_{n=0}^{+\infty} \int_{\tau}^{t}\left[a\left(r\left(s, w^{(1)}\right), \eta_{n}(s, \omega)\right)-a\left(r\left(s, w^{(1)}\right), \eta_{n-1}(s, \omega)\right)\right] d s, \\
\tau \leq t \leq \sigma_{r_{0}}\left(w^{(1)}\right),
\end{array}
$$

is uniformly convergent in $t, \tau \leq t \leq \sigma_{r_{0}}\left(w^{(1)}\right)$, with $\widetilde{P}$-measure 1 by Borel-Cantelli's lemma. Since, for $\lambda_{n}=K_{1}^{n-1} G B_{r_{0}}^{2}\left(\sigma_{r_{0}}\right)^{n} / n$ !,

$$
\begin{aligned}
& \tilde{P}\left\{\operatorname{Sup}_{\tau \leq \iota \leq r_{0}}\left|\int_{\tau}^{t}\left[b\left(r(s), \eta_{n}(s, \omega)\right)-b\left(r(s), \eta_{n-1}(s, \omega)\right)\right] d B(s)\right| \geq \lambda_{n}^{1 / 4}\right\} \\
& \quad \leq\left(\lambda_{n}^{1 / 4}\right)^{-2} \widetilde{E}\left\{\left[\int_{\tau}^{\sigma_{r_{0}}}\left(b\left(r(s), \eta_{n}(s, \omega)\right)-b\left(r(s), \eta_{n-1}(s, \omega)\right)\right) d B(s)\right]^{2}\right\} \\
& \quad \leq \lambda_{n}{ }^{1 / 2},
\end{aligned}
$$

we can prove, in the same way as before that, for any fixed $w^{(1)} \in W_{r_{0}}^{(1)}$,

$$
\begin{array}{r}
\sum_{n=1}^{+\infty} \int_{\tau}^{t}\left[b\left(r\left(s, w^{(1)}\right), \eta_{n}(s, \omega)\right)-b\left(r\left(s, w^{(1)}\right), \eta_{n-1}(s, \omega)\right)\right] d B\left(s, w^{(2)}\right), \\
\tau \leq t \leq \sigma_{r_{0}}\left(w^{(1)}\right),
\end{array}
$$

is uniformly convergent in $t, \tau \leq t \leq \sigma_{r_{0}}\left(w^{(1)}\right)$, with $\widetilde{P}$-measure 1 .

Similarily for any fixed $w^{(1)} \in W_{r_{0}}^{(1)}$,

$$
\begin{aligned}
& \sum_{n=1}^{+\infty} \int_{\mathrm{t}\left(\tau, w^{(1)}\right)}^{\mathrm{t}(t, w(1))}\left[m\left(\eta_{n}\left(\mathrm{t}^{-1}\left(s, w^{(1)}\right), \omega\right)\right)-m\left(\eta_{n-1}\left(\mathrm{t}^{-1}\left(s, w^{(1)}\right), \omega\right)\right)\right] d s, \\
& \sum_{n=1}^{+\infty} \int_{\mathrm{t}\left(\tau, w^{(1)}\right)}^{\mathrm{t}\left(t, w^{(1)}\right)}\left[\sigma\left(\eta_{n}\left(\mathrm{t}^{-1}\left(s, w^{(1)}\right), \omega\right)\right)-\sigma\left(\eta_{n-1}\left(\mathrm{t}^{-1}\left(s, w^{(1)}\right), \omega\right)\right)\right] d g\left(s, w^{(3)}\right), \\
& \sum_{n=1}^{+\infty} \int_{\mathrm{t}\left(\tau, w^{(1)}\right)}^{\mathrm{t}\left(t, w^{(1)}\right)} \int_{|\leq| u \mid<+\infty}\left[\beta\left(u, \eta_{n}\left(\mathrm{t}^{-1}\left(s, w^{(1)}\right), \omega\right)\right)\right. \\
& -\beta\left(u, \eta_{n-1}\left(\mathrm{t}^{-1}\left(s, w^{(1)}\right),\right.\right. \\
& \sum_{n=1}^{+\infty} \int_{\mathrm{t}\left(\tau, w^{(1)}\right)}^{\mathrm{t}\left(t, w^{(1)}\right)} \int_{|u|<+\infty}\left[\beta\left(u, \eta_{n}\left(\mathrm{t}^{-1}\left(s, w^{(1)}\right), \omega\right)\right)\right] d s d u / u^{2}, \\
& \left.-\beta\left(u, \eta_{n-1}\left(\mathrm{t}^{-1}\left(s, w^{(1)}\right), \omega\right)\right)\right] q\left(d s d u, w^{(3)}\right),
\end{aligned}
$$

are uniformly convergent in $t, \tau \leq t \leq \sigma_{r_{0}}\left(w^{(1)}\right)$, with $\widetilde{P}$-measure 1 .

Consequently combining (2.9) with (2.7) and (2.8) we see that, for any fixed $w^{(1)} \in W_{r_{0}}^{(1)}, \eta_{n}(t, \omega), \tau \leq t<+\infty$, is also uniformly convergent in $t, \tau \leq t<+\infty$, with $\widetilde{P}$-measure 1 . We denote this limit by $\eta(t, \omega)$.

Thus $\eta(t, \omega)$ belongs to $d_{1}$-class with $P$-measure 1 , and so measurable in $(t, \omega)$, since it is so the case with $\eta_{n}(t, \omega)$, as is 
recursively proved. By letting $n \rightarrow+\infty$ in (2.2), we can easily see that $\eta(t, \omega)$ is a solution of the stochastic integral equation (2.1) in the case $C\left(\omega^{(4)}\right)=C_{N}\left(\omega^{(4)}\right)$.

Thus the existence of the solution of our stochastic integral equation under the restriction $\left|C\left(\omega^{(4)}\right)\right| \leq N$ was completely proved. Once this is done, we can prove our lemma exactly in the same way as in K. Ito's [12] paper and we shall omit the rest of the proof.

It is to be noted here that $\{\eta(t, \omega), s \leq t<+\infty\}$, is a Borel measurable function of $\left\{\eta(s, \omega), r\left(t^{\prime}, w_{s}^{(2)+}\right), l\left(\mathrm{t}\left(t^{\prime}, w_{s}^{(1)+}\right), w_{\mathrm{t}(s, w}^{(3)+} w^{(1)}\right)\right)-$ $l\left(0, w_{\mathrm{t}\left(s, w^{(1)}\right)}^{(3)}\right)$ and $\left.B\left(t^{\prime}, w_{s}^{(2)+}\right)-B\left(0, w_{s}^{(2)+}\right), 0 \leq t^{\prime}<t-s\right\}$; this follow from

$$
\begin{aligned}
& \eta(t, \omega)=\eta(s, \omega)+\int_{s \wedge \sigma_{r_{0}}\left(w^{(1)}\right)}^{t \wedge \sigma_{r(\omega(1))}} a\left(r\left(t^{\prime}, w^{(1)}\right), \eta\left(t^{\prime}, \omega\right)\right) d t^{\prime} \\
& +\int_{s \wedge \sigma_{r_{0}}\left(w^{(1)}\right)}^{t \wedge \sigma_{r}\left(w^{(1)}\right)} b\left(r\left(t^{\prime}, w^{(1)}\right), \eta\left(t^{\prime}, \omega\right)\right) d B\left(t^{\prime} w^{(2)}\right) \\
& +\int_{\mathrm{t}\left(s \wedge \sigma_{r_{0}}\left(w^{(1)}\right), w^{(1)}\right)}^{\mathrm{t}\left(t \wedge \sigma_{r^{\prime}}\left(w^{(1)}\right), w(1)\right)} m\left(\eta\left(\mathrm{t}^{-1}\left(t^{\prime}, w^{(1)}\right), \omega\right)\right) d t^{\prime} \\
& +\int_{\mathrm{t}\left(s \wedge \sigma_{r_{0}}\left(w^{(1)}\right), w^{(1)}\right)}^{\left.\mathrm{t} t \boldsymbol{t} \wedge r_{0}\left(w^{(1)}\right), w^{(1)}\right)} \sigma\left(\eta\left(\mathrm{t}^{-1}\left(t^{\prime}, w^{(1)}\right), \omega\right)\right) d g\left(t^{\prime}, w^{(3)}\right) \\
& +\int_{\mathrm{t}\left(s \wedge \sigma_{r_{0}}\left(w^{(1)}\right), w^{(1)}\right)}^{\left.\left.\mathrm{t} t \boldsymbol{t} \wedge \sigma_{r} w^{(1)}\right) w^{(1)}\right)} \int_{|\leq| u \mid<+\infty} \beta\left(u, \eta\left(\mathrm{t}^{-1}\left(t^{\prime}, w^{(1)}\right), \omega\right)\right) p\left(d t^{\prime} d u, w^{(3)}\right) \\
& +\int_{\mathrm{t}\left(s \wedge \sigma_{r_{0}}\left(w^{(1)}\right), w^{(1)}\right)}^{\left.\mathrm{t} t \boldsymbol{t} \sigma_{r}\left(w^{(1)}\right), w^{(1)}\right)} \int_{|u|<1} \beta\left(u, \eta\left(\mathrm{t}^{-1}\left(t^{\prime}, w^{(1)}\right), \omega\right)\right) q\left(d t^{\prime} d u, w^{(3)}\right)
\end{aligned}
$$

In the following discussion, let $\eta(t,(r, \theta), \omega)$ be a solution of the equation (2.1) satisfying the following conditions: (1) $\tau=0$, (2) $C\left(\omega^{(4)}\right)=\theta$ and (3) $P_{r}\left\{r\left(t, w^{(1)}\right)=r\right\}=1$. (For convenience we shall write $\eta(t, \omega)$ sometimes instead of $\eta(t,(r, \theta), \omega))$. Here without loss of generality we may assum that $\eta(t,(r, \theta), \omega), 0 \leq t<+\infty$, is right continuous in $t$ for fixed $(r, \theta)$ and $\omega$.

Let $\left\{\widetilde{W}^{(1)}, r\left(t, \widetilde{w}^{(1)}\right), \tilde{\mathcal{B}}^{(1)}, \widetilde{P}_{r}^{(1)}, r \in R^{1}\right\}$ be a Bessel process and $\left\{\tilde{W}^{(2)}, B\left(t, \widetilde{w}^{(2)}\right), \tilde{\mathscr{B}}^{(2)}, \tilde{P}_{r}^{(2)}, r \in R^{1}\right\}$ a Brownian motion on $R^{1}$, and consider the probability space $\left\{\hat{\Omega}, \hat{\mathcal{B}}, P_{r}, r \in\left[r_{0}, 1\right]\right\}$, where $\hat{\Omega}=\Omega \times \tilde{W}^{(1)} \times \tilde{W}^{(2)}, \hat{\mathcal{B}}=\mathscr{B} \times \tilde{\mathscr{B}}^{(1)} \times \tilde{\mathscr{B}}^{(2)}$ and $\hat{P}_{r}=P_{1} \times \tilde{P}_{r}^{(1)} \times \tilde{P}_{0}^{(2)}$.

Using the method of successive approximations as above we can obtain the solution $\eta(t,(r, \theta), \widetilde{\widetilde{\omega}})$ of the following equations : 


$$
\eta(t,(r, \theta), \widetilde{\widetilde{\omega}})=\theta+\int_{0}^{t \wedge \sigma_{\left(r_{0}, 1\right)}^{\left(\widetilde{w}^{(1)}\right)}} a\left(r\left(s, \widetilde{w}^{(1)}\right), \eta(s,(r, \theta), \widetilde{\widetilde{\omega}})\right) d s
$$

$$
\begin{aligned}
& +\int_{0}^{\left.t \wedge \sigma_{\left(r_{0}, 1\right)} \tilde{w}^{(1)}\right)} b\left(r\left(s, \widetilde{w}^{(1)}\right), \eta(s,(r, s), \widetilde{\widetilde{\omega}})\right) d B\left(s, w^{(2)}\right), \\
& \quad \text { for } 0 \leq t<+\infty, \text { and } \widetilde{\widetilde{\omega}} \in \widetilde{\Omega}=\tilde{W}^{(1)} \times \widetilde{W}^{(2)},
\end{aligned}
$$

where $\widetilde{P}_{r}^{(1)}\left\{r\left(t, \widetilde{w}^{(1)}\right)=r\right\}=1$.

Without loss of generality we can also assume that $\eta(t,(r, \theta), \widetilde{\varpi})$ is continuous in $t, 0 \leq t<+\infty$, for any fixed $(r, \theta)$ and $\widetilde{\varpi}=\left(\widetilde{w}^{(1)}, \widetilde{w}^{(2)}\right)$.

Our diffusion desired will be now constructed as follows. Starting from the interior of the annulus, it will perform the motion given in the polar coordinate by the pair of $r\left(t, \widetilde{w}^{(1)}\right)$ and the solution $\eta(t, \widetilde{\widetilde{\omega}})$ of $(2.11)$ before $\sigma_{\left(r_{0}, 1\right)}\left(\widetilde{w}^{(1)}\right)$, and then it will stop for ever or perform the motion given by the pair of $r\left(t, w^{(1)}\right)$ and the solution of (2.1) according as it is on $\partial D_{r_{0}}^{(1)}=\left\{(r, \theta) ; r=r_{0}\right\}$ or on $\partial D$ at time $\sigma_{\left\{r_{0}, 1\right\}}\left(\widetilde{w}^{(1)}\right)$.

We shall express this procedure precisely in formulas. Consider a stochastic process $\{x(t,(r, \theta), \hat{\omega})(\equiv(r(t, r, \hat{\omega}), \eta(t,(r, \theta), \hat{\omega})))$, $0 \leq t<+\infty\}$ over a probability space $\left\{\hat{\Omega}, \hat{\mathscr{B}}, \hat{P}_{r}, r \in\left[r_{0}, 1\right]\right\}$ (for simplicity it is sometimes written as $\{x(t, \hat{\omega})(\equiv(r(t, \hat{\omega}), \eta(t, \hat{\omega})))$, $0 \leq t<+\infty\}$ ) defined as follows

(1) if $\sigma_{1}\left(\widetilde{w}^{(1)}\right)>\sigma_{r_{0}}\left(\widetilde{w}^{(1)}\right)$,

(2. 12) $(r(t, r, \hat{\omega}), \eta(t,(r, \theta), \hat{\omega}))=\left(r\left(t \wedge \sigma_{r_{0}}\left(\widetilde{w}^{(1)}\right), \widetilde{w}^{(1)}\right), \quad \eta(t,(r, \theta), \widetilde{\widetilde{\omega}})\right)$,

$$
0 \leq t<+\infty \text {, }
$$

(2) if $\sigma_{1}\left(\widetilde{w}^{(1)}\right)<\sigma_{r_{0}}\left(\widetilde{w}^{(1)}\right)$,

$$
\begin{aligned}
& (r(t, r, \hat{\omega}), \eta(t,(r, \theta), \hat{\omega}))=\left(r\left(t, \widetilde{w}^{(1)}\right), \eta(t,(r, \theta), \widetilde{\varpi})\right), \\
& 0 \leq t<\sigma_{1}\left(\widetilde{w}^{(1)}\right) \text {, } \\
& =\left(r\left(\left(t-\sigma_{1}\left(\widetilde{w}^{(1)}\right)\right) \wedge \sigma_{r_{0}}\left(w^{(1)}\right), w^{(1)}\right), \eta\left(\left(t-\sigma_{1}\left(\widetilde{w}^{(1)}\right)\right) \wedge \sigma_{r_{0}}\left(\widetilde{w}^{(1)}\right),\right.\right. \\
& \left.\left.\left(1, \eta\left(\sigma_{1}\left(\widetilde{w}^{(1)}\right),(r, \theta), \widetilde{\widetilde{\omega}}\right)\right), \omega\right)\right) \text {, } \\
& \sigma_{1}\left(\widetilde{w}^{(1)}\right) \leq t<+\infty
\end{aligned}
$$

where $(r, \theta) \in \bar{D}_{r_{0}}$.

Let $W$ be the space of path functions taking values in $\bar{D}_{r_{0}}$, and define a system of measures $P_{(r, \theta)}(*)$ over $\mathscr{B}$ by

$$
P_{(r, \theta)}(B)=\hat{P}_{r}\{\hat{\omega} ; x(\cdot,(r, \theta), \hat{\omega}) \in B\}, \text { for any } B \in \mathcal{B} \text {. }
$$


Then

$$
\mathfrak{M}=\left\{W, x(t, w), \mathscr{B}, P_{(r, \theta)},(r, \theta) \in \bar{D}_{r_{0}}\right\}
$$

is the diffusion satisfying the boundary condition (0.4). Though we shall prove this fact in $\S 5$, we shall here prove the following theorem by means the methods similar to those in K. Ito [13].

THEOREM 2.1. $\mathfrak{M}$ is a Markov process satisfying the following properties :

1) the semi-group $\left\{T_{t}, t \geq 0\right\}$ is strongly continuous, and

2) $T_{t} f(r, \theta)$ is continuous on $\bar{D}_{r_{0}}$, if $f$ is in $\mathcal{C}\left(\bar{D}_{r_{0}}\right)$.

PROOF. Let us observe first that $\mathfrak{M}$ is a Markov process. (2.10) and (2.11) show that

a) if $s<t \leq \sigma_{1}\left(\widetilde{w}^{(1)}\right), \eta(t, \hat{\omega})-\eta(s, \hat{\omega})$ is a Borel measurable of $r\left(t^{\prime}, \widetilde{w}_{s}^{(1)+}\right)$ and $B\left(t^{\prime}, \widetilde{w}_{s}^{(2)+}\right)-B\left(0, \widetilde{w}_{s}^{(2)+}\right), 0 \leq t^{\prime}<t-s$,

b) if $s \leq \sigma_{1}\left(\widetilde{w}^{(1)}\right)<t, \eta(t, \hat{\omega})-\eta(s, \hat{\omega})$ is a Borel measurable function of $r\left(t^{\prime} \wedge \sigma_{1}\left(\widetilde{w}_{s}^{(1)+}\right), \widetilde{w}_{s}^{(1)+}\right), B\left(t^{\prime} \wedge \sigma_{1}\left(\widetilde{w}_{s}^{(1)+}\right), \widetilde{w}_{s}^{(2)+}\right)-B\left(0, \widetilde{w}_{s}^{(2)+}\right)$, $r\left(\left(t^{\prime}-\sigma_{1}\left(\widetilde{w}_{s}^{(1)+}\right)\right) \vee 0, w^{(1)}\right), \quad B\left(\left(t^{\prime}-\sigma_{1}\left(\widetilde{w}_{s}^{(1)+}\right)\right) \vee 0, w^{(2)}\right)-B\left(0, w^{(2)}\right) \quad$ and $l\left(\mathrm{t}\left(\left(t^{\prime}-\sigma_{1}\left(\widetilde{w}_{s}^{(1)+}\right)\right) \vee 0, w^{(1)}\right), w^{(3)}\right)-l\left(0, w^{(3)}\right), 0 \leq t^{\prime}<t-s$, and

c) if $\sigma_{1}\left(\widetilde{w}^{(1)}\right)<s<t, \eta(t, \hat{\omega})-\eta(s, \hat{\omega})$ is a Borel measurable function of $r\left(t^{\prime}, w_{s}^{(1)+}\right), B\left(t^{\prime}, w_{s}^{(2)+}\right)-B\left(0, w_{s}^{(2)+}\right)$ and $l\left(\mathrm{t}\left(t^{\prime}, w_{s}^{(1)+}\right)\right.$, $\left.\left.w_{\mathrm{t}\left(s, w^{(1)}\right)}^{(3)+}\right)\right)-l\left(0, w_{\mathrm{t}\left(8, w^{(1)}\right)}^{(3)+}\right), 0 \leq t^{\prime} \leq t-s$. Now noting that $a(r, \theta)$, $b(r, \theta), m(\theta), \sigma(\theta)$ and $\beta(u, \theta)$ are periodic in $\theta$ with the period $2 \pi$, we can prove that for any $B \in \mathscr{B}_{s}$,

$$
\begin{aligned}
& \hat{E}_{r}\{f(x(t,(r, \theta), \hat{\omega})) ; B\} \\
&=\hat{E}_{r}\left\{B ; s<\sigma_{1}\left(\widetilde{w}^{(1)}\right) ;\right. \hat{E}_{r(s, r, \hat{\omega})}\{f(x(t-s,(r(s, r, \hat{\omega}), \\
&\left.\left.\eta(s,(r, \theta), \hat{\omega})), \hat{\omega})) ; t-s \leq \sigma_{1}\left(\widetilde{w}^{(1)}\right)\right\}\right\} \\
&+ \hat{E}_{r}\left\{B ; s<\sigma_{1}\left(\widetilde{w}^{(1)}\right) ;\right. \\
& \hat{E}_{r(s, r, \hat{\omega})}\{f(x(t-s,(r(s, r, \hat{\omega}), \\
&\left.\left.\eta(s,(r, \theta), \hat{\omega})), \hat{\omega})) ; \sigma_{1}\left(\widetilde{w}^{(1)}\right)<t-s\right\}\right\} \\
&+\hat{E}_{r}\left\{B ; \sigma_{1}\left(\widetilde{w}^{(1)}\right) \leqslant s ;\right. \hat{E}_{r(s, r, \hat{\omega})}\{f(x(t-s,(r(s, r, \hat{\omega}), \\
&\eta(s,(r, \theta), \hat{\omega})), \hat{\omega}))\}\}
\end{aligned}
$$




$$
\begin{aligned}
& =\hat{E}_{r}\left\{B ; s<\sigma_{1}\left(\widetilde{w}^{(1)}\right) ; \hat{E}_{r(s, r, \hat{\omega})}\{f(x(t-s,(r(s, r, \hat{\omega}),\right. \\
& \eta(s,(r, \theta), \hat{\omega})), \hat{\omega}))\}\} \\
& +\hat{E}_{r}\left\{B ; \sigma_{1}\left(\widetilde{w}^{(1)}\right) \leqslant s ; \hat{E}_{r(s, r, \hat{\omega})}\{f(x(t-s,(r(s, r, \hat{\omega}),\right. \\
& \eta(s,(r, \theta), \hat{\omega})), \hat{\omega}))\}\} \\
& =\hat{E}_{r}\left\{B ; \hat{E}_{r(s, r, \hat{\omega})}\{f(x(t-s,(r(s, r, \hat{\omega}), \eta(s,(r, \theta), \hat{\omega})), \hat{\omega}))\}\right\} \text {, }
\end{aligned}
$$

which shows that $\mathfrak{M}$ is a Markov process.

Next we shall construct a suitable version of $\left\{\widetilde{W}^{(1)}, r\left(t, \widetilde{w}^{(1)}\right)\right.$, $\left.\check{\mathcal{B}}^{(1)}, \tilde{P}_{r}^{(1)}, r \in R^{1}\right\}$. Given a Brownian motion $\left\{\tilde{W}^{(1)^{\prime}}, B\left(t, \widetilde{w}^{(1)^{\prime}}\right)\right.$, $\left.\tilde{\mathscr{B}}^{(1)^{\prime}}, \widetilde{P}_{r}^{(1)^{\prime}}, r \in R^{1}\right\}$ on $R^{1}$, we can define a increasing function $\mathfrak{s}\left(t, r, \widetilde{w}^{(1)^{\prime}}\right)$ by

$$
\mathfrak{S}\left(t, r, \widetilde{w}^{(1)^{\prime}}\right)=\int_{0}^{t} \exp \left[2\left(B\left(\tau, \widetilde{w}^{(1)^{\prime}}\right)+s(r)\right)\right] d \tau .
$$

Let

$$
r\left(t, r, \widetilde{w}^{(1)^{\prime}}\right)=r \exp \left[B\left(\mathfrak{g}^{-1}\left(t, r, \widetilde{w}^{(1)^{\prime}}\right), \widetilde{w}^{(1)^{\prime}}\right)\right] .
$$

Then it follow that $\left\{r\left(t, r, \widetilde{w}^{(1)^{\prime}}\right), 0 \leq t<+\infty, \widetilde{P}_{0}^{(1)^{\prime}}\right\}$ is a version of $\left\{\widetilde{W}^{(1)}, r\left(t, \widetilde{w}^{(1)}\right), \tilde{\mathscr{B}}^{(1)}, \widetilde{P}_{r}^{(1)}, r \in R^{1}\right\}$. Hereafter in this proof we shall always use this version. Now we consider a probability space $\left\{\hat{\Omega}^{\prime}, \hat{\mathcal{B}}^{\prime}, \hat{P} \hat{P}^{\prime}\right\}$, where $\hat{\Omega}^{\prime}=\Omega \times \tilde{W}^{(1)^{\prime}} \times \tilde{W}^{(2)}, \tilde{\mathcal{B}}^{\prime}=\tilde{\mathcal{B}} \times \widetilde{\mathcal{B}}^{(1)^{\prime}} \times \widetilde{\mathcal{B}}^{(2)}$ and $\hat{P}^{\prime}=P_{0} \times \widetilde{P}_{0}^{(1)^{\prime}} \times \widetilde{P}_{0}^{(2)}$. Throughout this section $\left\{r\left(t, r, \widetilde{w}^{(1)^{\prime}}\right)\right.$, $\left.0 \leq t<+\infty, \widetilde{P}_{0}^{(1)^{\prime}}\right\}$ is denoted simply by $\left\{r\left(t, r, \widetilde{w}^{(1)}\right), 0 \leq t<+\infty\right.$, $\left.\tilde{P}_{0}^{(1)}\right\}$ and $\left\{\hat{\Omega}^{\prime}, \hat{\mathcal{B}}^{\prime}, \hat{P}^{\prime}\right\}$ by $\{\hat{\Omega}, \hat{\mathcal{B}}, \hat{P}\}$.

Let

$$
\begin{aligned}
& \sigma_{:(\hat{\omega}, r)}= \begin{cases}\inf \{t ; r(t, r, \hat{\omega})=1, t \geqq 0\} & , \text { if such } \mathrm{t} \text { exists, } \\
+\infty & , \text { if otherwise, }\end{cases} \\
& \sigma_{r_{0}}(\hat{\omega}, r)= \begin{cases}\inf \left\{t ; r(t, r, \hat{\omega})=r_{0}, t \geqq 0\right\}, & \text { if such } \mathrm{t} \text { exists, } \\
+\infty & , \text { if otherwise, }\end{cases} \\
& \sigma_{\left\{r_{0}, 1\right\}}(\hat{\omega}, r)=\sigma_{1}(\hat{\omega}, r) \wedge \sigma_{r_{0}}(\hat{\omega}, r) \text {. }
\end{aligned}
$$

Since $\sigma_{1}(\hat{\omega}, r)$ and $\sigma_{\left\{r_{0}, 1\right\}}(\hat{\omega}, r)$ are independent of $\omega$ and $\widetilde{w}^{(2)}$, we shall sometimes write $\sigma_{1}\left(\widetilde{w}^{(1)}, r\right)$ and $\sigma_{\left(r_{0}, 1\right)}\left(\widetilde{w}^{(1)}, r\right)$ instead of $\sigma_{1}(\hat{\omega}, r)$ and $\sigma_{\left(r_{0}, 1\right\}}(\hat{\omega}, r)$ respectively for the convenience of notation. Similarily $\sigma_{r_{0}}(\hat{\omega}, r)$ is independent of $\left(\tilde{\omega}, \widetilde{w}^{(2)}\right)$. Hence we shall sometimes write $\sigma_{r_{0}}\left(\left(w^{(1)}, \widetilde{w}^{(1)}\right), r\right)$ instead of $\sigma_{r_{0}}(\hat{\omega}, r)$. Here without loss of generality we may assume 


$$
\sigma_{1}\left(\widetilde{w}^{(1)}, r\right)<+\infty \quad \text { and } \quad \sigma_{\left\{r_{0}, 1\right\}}\left(w^{(1)}, r\right)<+\infty
$$

Next let

$\tau_{\left\{r_{0}, 1\right\}}\left(\widetilde{w}^{(1)^{\prime}}, r\right)= \begin{cases}\inf \left\{t ; B\left(t, \widetilde{w}^{(1)^{\prime}}\right)+s(r)=s\left(r_{0}\right) \text { or } s(1), t \geq 0\right\}, \\ & \text { if such } t \text { exists, } \\ +\infty & \text { if otherwise, }\end{cases}$

and

$\tau_{1}\left(\widetilde{w}^{(1)^{\prime}}, r\right)= \begin{cases}\inf \left\{t ; B\left(t, \widetilde{w}^{(1)^{\prime}}\right)+s(r)=s(1), t \geq 0\right\}, & \text { if such } \mathrm{t} \text { exists, } \\ +\infty, & \text { if otherwise. }\end{cases}$

Then it is easily seen that $\sigma_{1}(\hat{\omega}, r)=\mathfrak{g}\left(\tau\left(\widetilde{w}^{(1)^{\prime}}, r\right), r, \widetilde{w}^{(1)^{\prime}}\right)$ and $\sigma_{\left\{r_{0}, 1\right\}}(\hat{\omega}, r)=\mathfrak{g}\left(\tau_{\left\{r_{0}, 1\right\}}\left(\widetilde{w}^{(1)^{\prime}}, r\right), r, \widetilde{w}^{(1)^{\prime}}\right)$. Hence if $r_{n} \rightarrow r$, then (a) $r\left(t, r_{n}, \hat{\omega}\right) \rightarrow r(t, r, \hat{\omega}), \quad(\mathrm{b}) \sigma_{1}\left(\hat{\omega}, r_{n}\right) \rightarrow \sigma_{1}(\hat{\omega}, r)$ and (c) $\sigma_{\left\{r_{0}, 1\right\}}\left(\hat{\omega}, r_{n}\right) \rightarrow$ $\sigma_{\left\{r_{0}, 1\right\}}(\hat{\omega}, r)$.

Now shall consider the direct probuct probability space $\left\{\widetilde{\Omega}^{*}, \tilde{\mathcal{B}}^{*}, \tilde{P}^{*}\right\}$ of $\{\widetilde{\Omega}, \tilde{\mathcal{B}}, \tilde{P}\}$ and $\left\{\tilde{W}^{(2)}, \tilde{\mathscr{B}}^{(2)}, \tilde{P}^{(2)}\right\}$. By $(2.11)$, if $t \leq \sigma_{\left(r_{0}, 1\right\}}\left(\widetilde{w}^{(1)}, r\right)$, then, for any sufficient large $n$,

$$
\begin{aligned}
& \widetilde{E}^{*}\left\{\left|\eta(t,(r, \theta), \hat{\omega})-\eta\left(t,\left(r_{n}, \theta_{n}\right), \hat{\omega}\right)\right|^{2}\right\} \\
& \leq 3\left[\left(\theta-\theta_{n}\right)^{2}+A_{r_{0}}^{2} \sigma_{\left\{r_{0}, 1\right\}}\left(\widetilde{w}^{(1)}, r\right) \int_{0}^{t} \widetilde{E}^{*}\left\{\left|r(s, r, \hat{\omega})-r\left(s, r_{n}, \hat{\omega}\right)\right|^{2}\right.\right. \\
& \left.\quad+\left|\eta(s,(r, \theta), \hat{\omega})-\eta\left(s,\left(r_{n}, \theta_{n}\right), \hat{\omega}\right)\right|^{2}\right\} d s \\
& +B_{r_{0}}^{2} \int_{0}^{t} \widetilde{E}^{*}\left\{\left|r(s, r, \hat{\omega})-r\left(s, r_{n}, \hat{\omega}\right)\right|^{2}\right. \\
& \left.\left.\quad+\left|\eta(s,(r, \theta), \hat{\omega})-\eta\left(s,\left(r_{n}, \theta_{n}\right), \hat{\omega}\right)\right|^{2}\right\} d s\right] \\
& +\widetilde{E}^{*}\left\{I_{3}\left(n,(r, \theta),\left(r_{n}, \theta_{n}\right) ; \hat{\omega}\right)\right\},
\end{aligned}
$$

where $\varlimsup_{n \rightarrow+\infty} I_{3}\left(n,(r, \theta),\left(r_{n}, \theta_{n}\right) ; \hat{\omega}\right)=0$ for almost all $\hat{\omega}$.

Using (2.16) and Fatou's lemma, we have

$$
\begin{aligned}
& \varlimsup_{\left(r_{n}, \theta_{n}\right) \rightarrow(r, \theta)} \widetilde{E}^{*}\left\{\left|\eta(t,(r, \theta), \hat{\omega})-\eta\left(t,\left(r_{n}, \theta_{n}\right), \hat{\omega}\right)\right|^{2}\right\} \\
& \leq 3\left[B_{r_{0}}^{2}+A_{r_{0}}^{2} \sigma_{\left\{r_{0}, 1\right\}}\left(\widetilde{w}^{(1)}, r\right)\right] \int_{0\left(r_{n}, \theta_{n}\right) \rightarrow(r, \theta)}^{t} \varlimsup_{E^{*}\{\eta \eta(s,(r, \theta), \hat{\omega})} \\
& \left.\quad-\left.\eta\left(s,\left(r_{n}, \theta_{n}\right), \hat{\omega}\right)\right|^{2}\right\} d s, \text { for } t \leqslant \sigma_{\left\{r_{0}, 1\right\}}\left(\widetilde{w}^{(1)}, r\right),
\end{aligned}
$$

which implies and the left side is identically zero for almost all $\left(\widetilde{P}_{0}^{(1)^{\prime}}\right), \widetilde{w}^{(1)^{\prime}}$.

Next, if $\sigma_{r_{0}}\left(\hat{\omega}, r_{n}\right)>t>\sigma_{1}(\hat{\omega}, r)$, then there exists an integer $N$ such that 
(2.17) $\quad \sigma_{r_{0}}\left(\hat{\omega}, r_{n}\right)>t>\sigma_{1}\left(\hat{\omega}, r_{n}\right)$, for every $n \geq N$.

By (A. 2), (B. 2), (C. 2), (2.1) and (2.17), we can prove that if $\sigma_{r_{0}}\left(\left(w^{(1)}, \widetilde{w}^{(1)}\right), r\right)>t>\sigma_{1}\left(\widetilde{w}^{(1)}, r\right)$, then, for any $n \geq N$,

$\widetilde{E}^{*}\left\{\mid \eta\left(t-\sigma_{1}(\hat{\omega}, r),\left(1, \eta\left(\sigma_{1}(\hat{\omega}, r),(r, \theta), \hat{\omega}\right)\right), \hat{\omega}\right)\right.$

$\left.-\left.\eta\left(t-\sigma_{1}\left(\hat{\omega}, r_{n}\right),\left(1, \eta\left(\sigma_{1}\left(\hat{\omega}, r_{n}\right),\left(r_{n}, \theta_{n}\right), \hat{\omega}\right)\right), \hat{\omega}\right)\right|^{2}\right\}$

$\leq 12\left[\widetilde{E^{*}}\left\{\left|\eta\left(\sigma_{1}(\hat{\omega}, r),(r, \theta), \omega\right)-\eta\left(\sigma_{1}\left(\hat{\omega}, r_{n}\right),\left(r_{n}, \theta_{n}\right), \hat{\omega}\right)\right|^{2}\right\}\right.$

$+A_{r_{0}}^{2} \sigma_{r_{0}}\left(\left(w^{(1)}, \widetilde{w}^{(1)}\right), r\right) \int_{0}^{\left.t-\sigma_{1} \widetilde{w}^{(1)}, r\right)} \tilde{E}^{*}\left\{\mid \eta\left(s,\left(1, \eta\left(\sigma_{1}(\hat{\omega}, r),(r, \theta), \hat{\omega}\right)\right), \hat{\omega}\right)\right.$

$\left.-\left.\eta\left(s,\left(1, \eta\left(\sigma_{1}\left(\hat{\omega}, r_{n}\right),\left(r_{n}, \theta_{n}\right), \hat{\omega}\right)\right), \hat{\omega}\right)\right|^{2}\right\} d s$

$+B_{r_{0}}^{2} \int_{0}^{t-\sigma_{1}\left(\widetilde{w}^{(1)}, r\right)} \widetilde{E}^{*}\left\{\mid \eta\left(s,\left(1, \eta\left(\sigma_{1}(\hat{\omega}, r),(r, \theta), \hat{\omega}\right)\right), \hat{\omega}\right)\right.$

$\left.-\left.\eta\left(s,\left(1, \eta\left(\sigma_{1}\left(\hat{\omega}, r_{n}\right),\left(r_{n}, \theta_{n}\right), \hat{\omega}\right)\right), \hat{\omega}\right)\right|^{2}\right\} d s$

$+M^{2} \sigma_{r_{0}}\left(\left(w^{(1)}, \widetilde{w}^{(1)}\right), r\right) \int_{0}^{t\left(t-\sigma_{1}^{(\widetilde{w}(1), r), w(1))}\right.} \widetilde{E}^{*}\left\{\mid \eta\left(\mathrm{t}^{-1}\left(s, w^{(1)}\right),\left(1, \eta\left(\sigma_{1}(\hat{\omega}, r),(r, \theta), \hat{\omega}\right)\right), \hat{\omega}\right)\right.$

(2.18) $\left.\quad-\left.\eta\left(\mathrm{t}^{-1}\left(s, w^{(1)}\right),\left(1, \eta\left(\sigma_{1}\left(\hat{\omega}, r_{n}\right),\left(r_{n}, \theta_{n}\right), \hat{\omega}\right)\right), \hat{\omega}\right)\right|^{2}\right\} d s$

$+S^{2} \int_{0}^{t\left(t-\sigma_{1}(\widetilde{w}(1), r), w(1)\right)} \widetilde{E}^{*}\left\{\mid \eta\left(\mathrm{t}^{-1}\left(s, w^{(1)}\right),\left(1, \eta\left(\sigma_{1}(\hat{\omega}, r),(r, \theta), \hat{\omega}\right)\right), \hat{\omega}\right)\right.$

$\left.-\left.\eta\left(\mathrm{t}^{-1}\left(s, w^{(1)}\right),\left(1, \eta\left(\sigma_{1}\left(\hat{\omega}, r_{n}\right),\left(r_{n}, \theta_{n}\right), \hat{\omega}\right)\right), \hat{\omega}\right)\right|^{2}\right\} d s$

$+2\left(\sigma_{r_{0}}\left(\left(w^{(1)}, \widetilde{w}^{(1)}\right), r\right) F\right)^{2} \int_{0}^{t\left(t-\sigma_{1}(\widetilde{w}(1), r), w(1)\right)} \widetilde{E}^{*}\left\{\mid \eta\left(\mathrm{t}^{-1}\left(s, w^{(1)}\right),\left(1, \eta\left(\sigma_{1}(\hat{\omega}, r),(r, \theta), \hat{\omega}\right)\right), \hat{\omega}\right)\right.$

$\left.-\left.\eta\left(\mathrm{t}^{-1}\left(s, w^{(1)}\right),\left(1, \eta\left(\sigma_{1}\left(\hat{\omega}, r_{n}\right),\left(r_{n}, \theta_{n}\right), \hat{\omega}\right)\right), \hat{\omega}\right)\right|^{2}\right\} d s$

$+F^{2} \int_{0}^{t\left(t-\sigma_{1}(\tilde{w}(1), r), w(1)\right)} \widetilde{E}^{*}\left\{\mid \eta\left(\mathrm{t}^{-1}\left(s, w^{(1)}\right),\left(1, \eta\left(\sigma_{1}(\hat{\omega}, r),(r, \theta), \hat{\omega}\right)\right), \hat{\omega}\right)\right.$

$\left.-\left.\eta\left(\mathrm{t}^{-1}\left(s, w^{(1)}\right),\left(1, \eta\left(\sigma_{1}\left(\hat{\omega}, r_{n}\right),\left(r_{n}, \theta_{n}\right), \hat{\omega}\right)\right), \hat{\omega}\right)\right|^{2}\right\} d s$

$\left.+\widehat{E}^{*}\left\{I_{4}\left((r, \theta),\left(r_{n}, \theta_{n}\right) ; \hat{\omega}\right)\right\}\right]$.

Then since $\lim _{n \rightarrow+\infty} \mathrm{t}\left(t-\sigma_{1}\left(\widetilde{w}^{(1)}, r_{n}\right), w^{(1)}\right)=\mathrm{t}\left(t-\sigma_{1}\left(\widetilde{w}^{(1)}, r\right), w^{(1)}\right)$, we have $\varlimsup_{n \rightarrow+\infty} I_{4}\left((r, \theta),\left(r_{n}, \theta_{n}\right) ; \hat{\omega}\right)=0$, for almost all $(\hat{P}), \hat{\omega}$.

By (2.18) and Fatou's lemma, we have

$$
\begin{array}{r}
\varlimsup_{\left(r_{n}, \theta_{n}\right) \rightarrow(r, \theta)} \widetilde{E}^{*}\left\{\left|\eta(t,(r, \theta), \hat{\omega})-\eta\left(t,\left(r_{n}, \theta_{n}\right), \hat{\omega}\right)\right|^{2}\right\} \\
\leq K_{2} \int_{0}^{t-\sigma_{1}(\tilde{w}(1), r)} \varlimsup_{\left(r_{n}, \theta_{n}\right) \rightarrow(r, \theta)} \widetilde{E}^{*}\left\{\mid \eta\left(s,\left(1, \eta\left(\sigma_{1}\left(\hat{\omega}^{(1)}, r\right),(r, \theta), \hat{\omega}\right)\right), \hat{\omega}\right)\right. \\
\left.-\left.\eta\left(s,\left(1, \eta\left(\sigma_{1}\left(\widetilde{w}^{(1)}, r_{n}\right),\left(r_{n}, \theta_{n}\right), \hat{\omega}\right)\right), \hat{\omega}\right)\right|^{2}\right\} d s, \\
\text { for } \sigma_{r_{0}}\left(\left(w^{(1)}, \widetilde{w}^{(1)}\right), r\right)>t>\sigma_{1}\left(\widetilde{w}^{(1)}, r\right),
\end{array}
$$

where $K_{2}=12\left[A_{r_{0}}^{2} \sigma_{0}\left(\left(w^{(1)}, \widetilde{w}^{(1)}\right), 1\right)+B_{r_{0}}^{2}+M \sigma_{r_{0}}\left(\left(w^{(1)}, \widetilde{w}^{(1)}\right), 1\right)+S^{2}+\right.$ 
$\left.2\left(\sigma_{r_{0}}\left(\left(w^{(1)}, \widetilde{w}^{(1)}\right), 1\right) F\right)^{2}+F^{2}\right]$. On the other hand it is easily seen that for almost all $\left(w^{(1)}, \widetilde{w}^{(1)^{\prime}}\right)$,

$$
\begin{aligned}
& \varlimsup_{\left(r_{n}, \theta_{n}\right) \rightarrow(r, \theta)} \tilde{E}^{*}\left\{\mid \eta\left(t-\sigma_{1}(\hat{\omega}, r),\left(1, \eta\left(\sigma_{1}\left(\hat{\omega}, r_{n}\right),\left(r_{n}, \theta_{n}\right), \hat{\omega}\right)\right), \hat{\omega}\right)\right. \\
&-\eta\left(t-\sigma_{1}\left(\hat{\omega}, r_{n}\right),\right.\left.\left.\left(1, \eta\left(\sigma_{1}\left(\hat{\omega}, r_{n}\right),\left(r_{n}, \theta_{n}\right), \hat{\omega}\right)\right), \hat{\omega}\right)\left.\right|^{2}\right\}=0 \\
& \text { for } \sigma_{r_{0}}\left(\left(w^{(1)}, \widetilde{w}^{(1)}\right), r\right)>t>\sigma_{1}\left(\widetilde{w}^{(1)}, r\right) .
\end{aligned}
$$

Hence combining (2.18) with (2.19) we have

$$
\left.\varlimsup_{\left(r_{n}, \theta_{n}\right) \rightarrow(r, \theta)} \widetilde{E}^{*}\left\{\mid \eta^{\prime} t,(r, \theta), \hat{\omega}\right)-\left.\eta\left(t,\left(r_{n}, \theta_{n}\right), \hat{\omega}\right)\right|^{2}\right\}=0,
$$

if $\sigma_{r_{0}}\left(\left(w^{(1)}, \widetilde{w}^{(1)}\right), r\right)>t>\sigma_{1}\left(\widetilde{w}^{(1)}, r\right)$, for almost all $\left(w^{(1)}, \widetilde{w}^{(1)^{\prime}}\right)$.

And it is easily seen that if $t \geq \sigma_{r_{0}}\left(\left(w^{(1)}, \widetilde{w}^{(1)}\right), r\right)$, then (2.20) also holds.

Thus, (2.20) is true for any value of $t$ and it follows from this that if $\left(r_{n}, \theta_{n}\right) \rightarrow(r, \theta)$, there exists a subsequence $\left(r_{n_{k}}, \theta_{n_{k}}\right)$ such that

$$
\eta\left(t,\left(r_{n_{k}}, \theta_{n_{k}}\right), \hat{\omega}\right) \rightarrow \eta(t,(r, \theta), \hat{\omega}),
$$

for almost all $(\hat{P}), \hat{\omega}$. Therefore using Lebesque's bounded convergence theorem we can prove that if $\left(r_{n}, \theta_{n}\right) \rightarrow(r, \theta)$, then there exists a subsequece $\left(r_{n_{k}}, \theta_{n_{k}}\right)$ such that

$$
T_{t} f\left(r_{n_{k}}, \theta_{n_{k}}\right) \rightarrow T_{t} f(r, \theta), \text { for } f(r, \theta) \in \mathcal{C}\left(\bar{D}_{r_{0}}\right) \text {. }
$$

Since this is true for every sequence $\left(r_{n}, \theta_{n}\right) \rightarrow(r, \theta)$, we see that

$$
\lim _{\left(r^{\prime}, \theta^{\prime}\right) \rightarrow(r, \theta)} T_{t} f\left(r^{\prime}, \theta^{\prime}\right)=T_{t} f(r, \theta) \text {, for } f(r, \theta) \in \mathcal{C}\left(\bar{D}_{r_{0}}\right) \text {. }
$$

The right continuity of $x(t,(r, \theta), \hat{\omega})$ in $t$ implies that $T_{t}$, $t \geq 0$, is strongly continuous and Theorem 2.1 is therefore completely proved.

According to E. B. Dynkin and A. A. Yushkebich [8], Theorem 2.1 implies the following

Proposition 2.1. M has the strong Markov property.

3. Construction of path functions (2). In this section we shall construct the path functions of the diffusion in the unit disk whose generator and boundary condition are given by $(0.3)$ and 
(0.4) with “ $\delta(\theta) \equiv 0$ ". We shall use the same notations and definitions introduced in the previous section, except $\left\{W^{(1)}, r\left(t, w^{(1)}\right)\right.$, $\left.\mathscr{B}^{(1)}, P_{r}^{(1)}, r \in[0,1]\right\}$ and $\mathrm{t}\left(t, w^{(1)}\right)$ which will denote here a reflecting barrier Bessel process and its local time at point $\{1\}$.

Now we consider a stochastic integral equation of the same type as (2.1);

$$
\begin{aligned}
& \eta(t, \omega)=C\left(\omega^{(4)}\right)+\int_{\tau \wedge \sigma_{r_{0}}\left(w^{(1)}\right)}^{t \wedge \sigma_{r_{0}}{ }^{(w(1))}} a\left(r\left(s, w^{(1)}\right), \eta(s, \omega)\right) d s \\
& +\int_{\tau \wedge \sigma_{r_{0}\left(w^{(1)}\right)}}^{t \wedge \sigma_{r_{0}}(w(1))} b\left(r\left(s, w^{(1)}\right) \eta(s, \omega)\right) d B\left(s, w^{(2)}\right) \\
& +\int_{\mathrm{t}\left(\tau \wedge \sigma_{r_{0}}\left(w^{(1)}\right), w^{(1)}\right)}^{\left.\mathrm{t}\left(t \wedge \sigma_{r_{0}}{ }^{(w(1)}\right), w(1)\right)} m\left(\eta\left(\mathrm{t}^{-1}\left(s, w^{(1)}\right), \omega\right)\right) d s \\
& +\int_{\mathrm{t}\left(\tau \wedge \sigma_{r_{0}}\left(w^{(1)}\right), w^{(1)}\right)}^{\mathrm{t}\left(\mathrm{t} \wedge \sigma_{r_{0}}{ }^{(w(1)), w(1)}\right.} \sigma\left(\eta\left(\mathrm{t}^{-1}\left(s, w^{(1)}\right), \omega\right)\right) d g\left(s, w^{(3)}\right) \\
& +\int_{\mathrm{t}\left(\tau \wedge \sigma_{r_{0}}\left(w^{(1)}\right), w^{(1)}\right)}^{\mathrm{t}\left(t \wedge \sigma_{\left.\left.r^{(}\right)(w(1)), w(1)\right)}\right.} \int_{1 \leq|u|<+\infty} \beta\left(u, \eta\left(\mathrm{t}^{-1}\left(s, w^{(1)}\right), \omega\right)\right) p\left(d s d u, w^{(3)}\right) \\
& +\int_{\mathrm{t}\left(\tau \wedge \sigma_{r_{0}}\left(w^{(1)}\right), w^{(1)}\right)}^{\mathrm{t}\left(t \wedge \sigma_{\left.r^{(}\right)}(w(1)), w(1)\right)} \int_{|u|<1} \beta\left(u, \eta\left(\mathrm{t}^{-1}\left(s, w^{(1)}\right), \omega\right)\right) q\left(d s d u, w^{(3)}\right),
\end{aligned}
$$

for any $\tau \geq 0$ and $0<r_{0}<1$. Corresponding to Lemma 2.1 we have

LEMMA 3.1. Lemma 2.1 is also true in this case of $\delta(\theta) \equiv 0$.

Proof. First we note that there exists $W_{r_{0}}^{(1)}$ such that

$$
W_{r_{0}}^{(1)}=\left\{w^{(1)} ; w^{(1)} \in W^{(1)}, \sigma_{r_{0}}\left(w^{(1)}\right)<+\infty, \mathfrak{t}\left(\sigma_{r_{0}}\left(w^{(1)}\right), w^{(1)}\right)<+\infty\right\},
$$

and

$$
P_{r}^{(1)}\left(W_{r_{0}}^{(1)}\right)=1, \text { for any } r_{0} \leqslant r \leqslant 1
$$

Hence it is enough to prove that Lemma 3.1 hods for any $w^{(1)} \in W_{r_{0}}^{(1)}$.

In order to find a solution of (3.1) we make use of the method of successive approximations; we define $\eta_{n}(t, \omega)$, $n=0,1,2, \cdots$, exactly in the same way as in the case $\delta(\theta) \equiv 1$.

Now using the same methods as those in the proof of Lemma 2.1 , we can prove the both of $\widehat{E}\left\{\left(\eta_{1}(t, \omega)\right)^{2}\right\}$ and $\widetilde{E}\left\{\mid \eta_{1}(t, \omega)-\right.$ $\left.\left.\eta_{0}(t, \omega)\right|^{2}\right\}, \tau \leq t<+\infty$, are bounded and $\eta_{1}(t, \omega), \tau \leq t<+\infty$, is measurable in $(t, \omega)$. Furthermore for any fixed $w^{(1)} \in W_{r_{0}}^{(1)},\left\{\eta_{1}(t, \omega)\right.$, $l\left(\mathrm{t}\left(s, w_{\tau}^{(1)+}\right), w_{\mathrm{t}\left(\tau, w^{(1)}\right)}^{(3)+}\right)-l\left(0, w_{\mathrm{t}\left(\tau, w^{(1)}\right)}^{(3)+}, B\left(s, w_{\tau}^{(2)+}\right)-B\left(0, w_{\tau}^{(2)+}\right), 0 \leq s \leq t-\tau\right\}$ is independent of $\left\{l\left(\mathrm{t}\left(s, w_{t}^{(1)+}\right), w_{\mathrm{t}\left(t, w^{(1)}\right)}^{(3)+}\right)-l\left(0, w_{\mathrm{t}\left(t, w^{(1)}\right)}^{(3)+}, B\left(s, w_{t}^{(2)+}\right)-\right.\right.$ 
$B\left(0, w_{t}^{(2)+}\right), 0 \leq s \leq \sigma_{r_{0}}\left(w^{(1)}\right)-t$, for any $\tau \leq t \leq \sigma_{r_{0}}\left(w^{(1)}\right)$. Thus we can define $\eta_{2}(t, \omega)$ and so recursively $\eta_{n}(t, \omega), n=3,4, \cdots$. Now it follows for any $t, \tau \leq t \leq \sigma_{r_{0}}\left(w^{(1)}\right)$,

$$
\begin{aligned}
& \widetilde{E}\left\{\left[\int_{\tau}^{t}\left(a\left(r(s), \eta_{n}(s, \omega)\right)-a\left(r(s), \eta_{n-1}(s, \omega)\right)\right) d s\right]^{2}\right\} \\
& \quad \leq A_{r_{0}}^{2} \sigma_{r_{0}} \int_{\tau}^{t} \widetilde{E}\left\{\left|\eta_{n}(s, \omega)-\eta_{n-1}(s, \omega)\right|^{2}\right\} d s, \\
& \widetilde{E}\left\{\left[\int_{\tau}^{t}\left(b\left(r(s), \eta_{n}(s, \omega)\right)-b\left(r(s), \eta_{n-1}(s, \omega)\right)\right) d B(s)\right]^{2}\right\} \\
& \quad \leq B_{r_{0}}^{2} \int_{\tau}^{t} \widehat{E}\left\{\left|\eta_{n}(s, \omega)-\eta_{n-1}(s, \omega)\right|^{2}\right\} d s, \\
& \widetilde{E}\left\{\left[\int_{\mathrm{t}(\tau)}^{\mathrm{t}(t)}\left(m\left(\eta_{n}\left(\mathrm{t}^{-1}(s), \omega\right)\right)-m\left(\eta_{n-1}\left(\mathrm{t}^{-1}(s), \omega\right)\right)\right) d s\right]^{2}\right\} \\
& \quad \leq M^{2} \mathrm{t}\left(\sigma_{r_{0}}\right) \int_{\tau}^{t} \widetilde{E}\left\{\left|\eta_{n}(s, \omega)-\eta_{n-1}(s, \omega)\right|^{2}\right\} \mathrm{t}(d s), \\
& \widetilde{E}\left\{\left[\int_{\mathrm{t}(\tau)}^{\mathrm{t}(t)}\left(\sigma\left(\eta_{n}\left(\mathrm{t}^{-1}(s), \omega\right)\right)-\sigma\left(\eta_{n-1}\left(\mathrm{t}^{-1}(s), \omega\right)\right)\right) d g(s)\right]^{2}\right\} \\
& \quad \leq S^{2} \int_{\tau}^{t} \widetilde{E}\left\{\left|\eta_{n}(s, \omega)-\eta_{n-1}(s, \omega)\right|^{2}\right\} \mathrm{t}(d s), \\
& \widetilde{E}\left\{\left[\int_{\mathrm{t}(\tau)}^{\mathrm{t}(t)} \int_{|u|>1}\left(\beta\left(u, \eta_{n}\left(\mathrm{t}^{-1}(s), \omega\right)\right)-\beta\left(u, \eta_{n-1}\left(\mathrm{t}^{-1}(s), \omega\right)\right)\right) d u d s / u^{2}\right]^{2}\right\} \\
& \quad \leq 2 F^{2} \mathrm{t}\left(\sigma_{r_{0}}\right) \int_{\tau}^{t} \widetilde{E}\left\{\left|\eta_{n}(s, \omega)-\eta_{n-1}(s, \omega)\right|^{2}\right\} \mathrm{t}(d s),
\end{aligned}
$$

and

$$
\begin{aligned}
& \tilde{E}\left\{\left[\int_{\mathrm{t}(\tau)}^{\mathrm{t}(t)} \int_{|u|<+\infty}\left(\beta\left(u, \eta_{n}\left(\mathrm{t}^{-1}(s), \omega\right)\right)-\beta\left(u, \eta_{n-1}\left(\mathrm{t}^{-1}(s), \omega\right)\right)\right) q(d s d u)\right]^{2}\right\} \\
& \quad \leq F^{2} \int_{\tau}^{t} \widetilde{E}\left\{\left|\eta_{n}(s, \omega)-\eta_{n-1}(s, \omega)\right|^{2}\right\} \mathrm{t}(d s) .
\end{aligned}
$$

Hence we have that for any $t, \tau \leq t<+\infty$,

$$
\begin{aligned}
& \widetilde{E}\left\{\left|\eta_{n+1}(t, \omega)-\eta_{n}(t, \omega)\right|^{2}\right\} \\
& \quad \leq K_{3} \int_{\tau \wedge \sigma_{r 0}\left(w^{(1)}\right)}^{t \wedge \sigma_{r_{0}}{ }^{(w(1))}} \widetilde{E}\left\{\left|\eta_{n}(s, w)-\eta_{n-1}(s, \omega)\right|^{2}\right\} \hat{\mathfrak{t}}\left(d s, w^{(1)}\right),
\end{aligned}
$$

where $K_{3}=7\left[A_{r_{0}}^{2} \sigma_{r_{0}}\left(w^{(1)}\right)+B_{r_{0}}^{2}+M^{2} \mathrm{t}\left(\sigma_{r_{0}}\left(w^{(1)}\right), w^{(1)}\right)+S^{2}+2 F^{2} \mathrm{t}\left(\sigma_{r_{0}}\left(w^{(1)}\right)\right.\right.$, $\left.\left.w^{(1)}\right)+F^{2}\right]$, and $\hat{\mathrm{t}}\left(t, w^{(1)}\right)=t+\mathrm{t}\left(t, w^{(1)}\right), \tau \leq t<+\infty$.

Since

(3.2) $\int_{\tau}^{t} \int_{\tau}^{s_{1}} \ldots \int_{\tau}^{s_{n}} \hat{\mathrm{t}}\left(d s_{n}, w^{(1)}\right) \cdots \hat{\mathrm{t}}\left(d s_{1}, w^{(1)}\right)=\left(\int_{\tau}^{t} \hat{\mathrm{t}}\left(d s, w^{(1)}\right)\right)^{n} / n !<+\infty$, 
we can complete tne proof, repeating the same arguments as those in the proof of Lemma 2.1.

Now we define a combination $\mathfrak{M}=\left\{W, x(t, w), \mathscr{B}, P_{(r, \theta)},(r, \theta) \in\right.$ $\left.\bar{D}_{r_{0}}\right\}$ using the same procedures as those in Section 2.

Then corresponding to Theorem 2.1 and Proposition 2.1, we have

THEOREM 3.1. Theorem 2.1 is also true in this case of “ $\delta(\theta) \equiv 0 "$.

Now we have, as an immediate result from this,

PROPOSITION 3.1. M has the strong Markov property.

Repeating the same arguments as those in the proof of Theorem 2.1 and noting (3.2) we can prove Theorem 3.1. The proof of Proposition 3.1 is clear.

4. The Markov process on the boundary concerning the diffusion on $\overline{\boldsymbol{D}}_{r_{0}}$. In this section we shall derive a system $\left\{\mathfrak{M}^{(\alpha)}, \alpha \geq 0\right\}$ of Markov process on $\partial D$ from the diffusion $\mathfrak{M}$ on $\bar{D}_{r_{0}}$ constructed in Sections 2 and 3 , and use it to discuss the Green operator of $\mathfrak{M}$.

Let $\left\{W^{(1)^{\prime}}, B\left(t, \widetilde{w}^{(1)^{\prime}}\right), \mathfrak{B}^{(1)^{\prime}}, \widetilde{P}_{0}^{(1)^{\prime}}\right\}$ be a Brownian motion on $R^{1}$ introudced in Section 2. Now we define $\widetilde{r}\left(t, r, \widetilde{w}^{(1)^{\prime}}\right)$ by

$$
\begin{aligned}
\widetilde{r}\left(t, r, \widetilde{w}^{(1)^{\prime}}\right)=r+\frac{1}{2} \int_{0}^{t}\left(\widetilde{r}\left(s, r, \widetilde{w}^{(1)^{\prime}}\right)\right)^{-1} d s+B\left(t, \widetilde{w}^{(1)^{\prime}}\right), & \\
0 \leq t<+\infty & \text { and } 0<r \leq 1 .
\end{aligned}
$$

The $\left\{\widetilde{r}\left(t, r, \widetilde{w}^{(1)^{\prime}}\right), 0 \leq t \leq \sigma_{\left(r_{0}, 1\right)}\left(\widetilde{w}^{(1)^{\prime}}, r\right), \widetilde{P}_{0}^{(1)^{\prime}}\right\}$ is a version of $\left\{r\left(t, \widetilde{w}^{(1)}\right), 0 \leq t \leq \sigma_{\left\{r_{0}, 1\right\}}\left(\widetilde{w}^{(1)}, r\right), \widetilde{P}_{r}^{(1)}\right\}$, where

$$
\sigma_{\left(r_{0}, 1\right\}}\left(\widetilde{w}^{(1)^{\prime}}, r\right)= \begin{cases}\inf \left\{s ; \widetilde{r}\left(s, r, \widetilde{w}^{(1)^{\prime}}\right)=1 \text { or } r_{0}\right\}, & \text { if such } t \text { exists, } \\ +\infty & , \text { if otherwise. }\end{cases}
$$

Noting that the infinitesimal generator $\mathbb{B S}$ of minimal diffusion corresponding to $\mathfrak{M}$ constructed in Sections 2 and 3 is a local operator in $D_{r_{0}}$, viewing (4.1) and (2.11) as the stochastic integral equation for a two dimensional diffusion $(r(t, \hat{\omega}), \eta(t, \hat{\omega}))$ and using Theorem 3.2 in K. lto [11] we have the following 
PROPOSITION 4.1. We assume that $a(r, \theta)$ and $b(r, \theta)$ belong to $\mathcal{C}^{1}\left(\bar{D}_{r_{0}}\right)$. If $u(r, \theta)$ belongs to $\mathcal{C}^{2}\left(D_{r_{0}}\right)$, then

$$
\begin{aligned}
& \lim _{t \downarrow 0}[\left.T_{t} u(r, \theta)-u(r, \theta)\right] / t \\
&=\frac{1}{2}\left(\frac{\partial^{2}}{\partial r^{2}}+\frac{1}{r} \frac{\partial}{\partial r}+b(r, \theta) \frac{\partial^{2}}{\partial \theta^{2}}+2 a(r, \theta) \frac{\partial}{\partial \theta}\right) u(r, \theta), \\
& \text { for }(r, \theta) \in D_{r_{0}},
\end{aligned}
$$

where $T_{t}$ is the semi-group of $\mathfrak{M}$ constructed in Section 2 or Section 3.

The proof is easy and so is omitted.

From now on we shall always impose the following:

Assumpution A : $a(r, \theta)$ and $b(r, \theta)$ belong to $\mathcal{C}^{3}\left(\bar{D}_{r_{0}}\right)$.

Throughout Sections 4 and 5, though some statements in the sequel will be also true without Assumption $A$.

Here, we cite some known results in the theory of differential equations for late use.

Lemma 4.1.8) 1) For any $\alpha \geq 0$, the equation

$$
(\alpha-A) u(r, \theta)=0, \quad \text { on } D_{r_{0}}
$$

with the boundary condition

$$
u(r, \theta)=f(r, \theta), \quad \text { on } \quad \partial D_{r_{0}}, \quad f \in \mathcal{C}\left(\partial D_{r_{0}}\right),
$$

has a unique solution $u(r, \theta) \in \mathcal{C}\left(\bar{D}_{r_{0}}\right)$, which is expressible in the form

$$
u(r, \theta)=h^{\alpha} f(r, \theta)=h_{1}^{\alpha} f(r, \theta)+h_{2}^{\alpha} f(r, \theta),
$$

where

$$
\begin{aligned}
& h_{1}^{\alpha} f(r, \theta)=\int_{\partial D} h_{1}^{\alpha}\left((r, \theta) ; d r^{\prime} d \theta^{\prime}\right) f\left(r^{\prime}, \theta^{\prime}\right), \\
& h_{2}^{\alpha} f(r, \theta)=\int_{\partial D_{r_{0}}^{(1)}} h_{2}^{\alpha}\left((r, \theta) ; d r^{\prime} d \theta^{\prime}\right) f\left(r^{\prime}, \theta^{\prime}\right),
\end{aligned}
$$

and $h_{i}^{\alpha}\left((r, \theta) ; d r^{\prime} d \theta^{\prime}\right), i=1,2$, are the positive measure on $\partial D$ and $\partial D_{r_{0}}^{(1)}$ respectively such that $h_{1}^{\alpha}((r, \theta): \partial D)+h_{2}^{\alpha}\left((r, \theta) ; \partial D_{r_{0}}^{(1)}\right) \leq 1$.

2) If $f \in \mathcal{C}^{3}\left(\partial D_{r_{0}}\right)$, then $h^{\alpha} f \in \mathcal{C}^{2}\left(\bar{D}_{r_{0}}\right)$.

8) Cf. S. Ito [15] and T. Ueno [21]. 
LEMma 4.2.9) There is a system of linear operators $\left\{G_{\alpha}^{0}\right\}$ on the space $\mathcal{C}\left(\bar{D}_{r_{0}}\right)$, satisfying

$$
\begin{gathered}
\left\|G_{\alpha}^{0} f\right\| \leq \frac{1}{\alpha}\|f\|, \quad f \in \mathcal{C}\left(\bar{D}_{r_{0}}\right), \\
G_{\alpha}^{0} f-G_{\beta}^{0} f+(\alpha-\beta) G_{\alpha}^{0} G_{\beta}^{0} f=0, \quad f \in \mathcal{C}\left(\bar{D}_{r_{0}}\right), \\
(\alpha-A) G_{\alpha}^{0} f(r, \theta)=f(r, \theta), \quad(r, \theta) \in D_{r_{0}}, \quad f \in \mathcal{C}^{1}\left(\bar{D}_{r_{0}}\right), \\
G_{\alpha}^{0} f \in \tilde{C}\left(\bar{D}_{r_{0}}\right), \quad f \in \mathcal{C}\left(\bar{D}_{r_{0}}\right),
\end{gathered}
$$

where

$$
\begin{gathered}
\tilde{\mathcal{C}}\left(\bar{D}_{r_{0}}\right)=\left\{f \in \mathcal{C}\left(\bar{D}_{r_{0}}\right) ; f(r, \theta)=0 \text { on } \partial D_{r_{0}}\right\}, \\
G_{\alpha}^{0} f \in \tilde{\mathcal{C}}^{2}\left(D_{r_{0}}\right), \quad \text { for } f \in \mathcal{C}^{1}\left(\bar{D}_{r_{0}}\right), \\
\lim _{\alpha \rightarrow+\infty}\left\|\alpha G_{\alpha}^{0} f-f\right\|=0, \quad \text { for } f \in \tilde{\mathcal{C}\left(\bar{D}_{r_{0}}\right),} \\
\lim _{\alpha \rightarrow+\infty} \alpha G_{\alpha}^{0} f(x)=f(x), \quad \text { for } x \in D_{r_{0}}, f \in \mathcal{C}\left(\bar{D}_{r_{0}}\right) .
\end{gathered}
$$

Making use of Propositions 2.1,3.1 and 4.1 and Lemmas 4.1 and 4.2 , we have

Proposition 4.2. Let $M$ be a diffusion process constructed in Section 2 or 3 . Then

$$
G_{\alpha} f(r, \theta)=\widetilde{G}_{\alpha}^{0} f(r, \theta)+h_{1}^{\alpha}\left(G_{\alpha} f\right)(r, \theta), \quad f \in \mathcal{C}\left(\bar{D}_{r_{0}}\right),
$$

where $G_{c}$ is the Green operator corresponding to the diffusion $\mathfrak{M}$, and $\widetilde{G}_{\alpha}^{0} f=G_{\alpha}^{0} f+h_{2}^{\alpha} f$.

Using the strong Markov property of $\mathfrak{M}$, the proof is easy and so is omited.

We shall discuss only the diffusion $\mathfrak{M}=\left\{W, x(t, w), \mathfrak{B}, P_{(r, \theta)}\right.$, $\left.(r, \theta) \in \bar{D}_{r_{0}}\right\}$ constructed in Section 3, while we shall only state the final result as Theorem 4.2 for the diffusion constrncted in Section 2.

We shall first derive the Markov process $\mathfrak{M}^{(0)}$ on the boundary $\partial D$ from the dffusion $M i$ on $\bar{D}_{r_{0}}$. Let $W^{0}$ be the space of path functions with values in $\partial D$. And we define a system of probability measures $P_{\theta}^{0}(\cdot),(1, \theta) \in \partial D$, by

9) Cf. S. Ito [15] and T. Ueno [21]. 


$$
P_{\theta}^{0}\left(w^{0} \in B\right)=P_{(1, \theta)}\left(\theta\left(\mathfrak{t}^{-1}(t, w), w\right) \in B\right), \quad B \in \mathscr{B}^{0},
$$

where $\mathrm{t}^{-1}(t, w)=\inf \{s ; \mathrm{t}(s, w)>t\}$. Then we have

Proposition 4.3. $\mathfrak{M}^{(0)}=\left\{W^{0}, z\left(t, w^{0}\right), \mathscr{B}^{0}, P_{\theta}^{0},(1, \theta) \in \partial D\right\}$ is a strong Markov process satisfying

1) $T_{t}^{0}$ is strongly continuous, and

2) $\mathcal{C}(\partial D)$ is invariant under $T_{t}^{0}$, where $T_{t}^{0}, t \geq 0$, is the semi-group corresponding to $\mathfrak{M}^{(0)}$.

This fact was established by many authors even for more general Markov processes ${ }^{10)}$. We shall here sketch a proof which is due to K. Ito and H. P. McKean [14].

Proof. First we note that

$$
\mathrm{t}^{-1}(t+s, w)=\mathrm{t}^{-1}\left(t, w_{\mathrm{t}-1(s, w)}^{+}\right)+\mathrm{t}^{-1}(s, w) .
$$

Given $t \geq 0$,

$$
\left\{w ; \mathrm{t}^{-1}(s, w) \geq t\right\}=\{w ; s \geq \mathrm{t}(t, w)\} \cap\left\{w ; \mathrm{t}^{-1}(+\infty, w)\right\} \in \mathscr{B}_{t} .
$$

Hence we have, for Borel measurable $g$ and $f$,

$$
\begin{aligned}
E_{\theta}^{0} & \left(f\left(w_{s}^{0-}\right) g\left(z\left(t+s, w^{0}\right)\right)\right) \\
& =E_{(1, \theta)}\left\{f\left(\theta\left(\mathrm{t}^{-1}\left(\cdot, w_{\mathrm{t}^{-1}(s, w)}^{-1}\right), w_{\mathrm{t}^{-1}(s, w)}^{-}\right)\right) g\left(\theta\left(\mathrm{t}^{-1}\left(t, w_{\mathrm{t}^{-1}(s, w)}^{+}\right), w_{\mathrm{t}-1}^{+}(s, w)\right)\right)\right\} \\
& =E_{(1, \theta)}\left\{f\left(\theta\left(\mathfrak{t}^{-1}\left(\cdot, w_{\mathrm{t}^{-1}(s, w)}^{-}\right), w_{\mathrm{t}}^{-}{ }^{-1}(s, w)\right)\right) E_{\left(1, \theta\left(\mathrm{t}^{-1}(s, w) w\right)\right.}\left\{g\left(\theta\left(\mathrm{t}^{-1}(t, w), w\right)\right)\right\}\right\} \\
& =E_{\theta}^{0}\left\{f\left(w_{s}^{0-}\right) E_{z\left(s, w^{0}\right)}^{0}\left\{g\left(z\left(t, w^{0}\right)\right)\right\}\right\}, \quad t, s \geq 0 .
\end{aligned}
$$

Hence $\mathfrak{M}^{(0)}$ has the Markov property.

On the other hand, using the same procedure as those of Section 2 , it is easily seen that there exists a version $\left\{\eta\left(\mathrm{t}^{-1}\left(s, w^{(1)}\right)\right.\right.$, $(1, \theta), \hat{\omega}), 0 \leq s<+\infty\}$ of $\mathfrak{M g}^{(0)}$ satisfying

$$
\begin{aligned}
& \lim _{\left(1, \theta^{\prime}\right) \rightarrow(1, \theta)} E\left\{f\left(\eta\left(\mathrm{t}^{-1}\left(s, w^{(1)}\right),\left(1, \theta^{\prime}\right), \omega\right)\right)\right\} \\
&=E\left\{f\left(\eta\left(\mathrm{t}^{-1}\left(s, w^{(1)}\right),(1, \theta), \omega\right)\right)\right\}, \quad \text { for } f \in \mathcal{C}(\partial D) .
\end{aligned}
$$

Hence using the same method as those in Sections 2 and 3 we can prove 1) and 2). According to E. B. Dynkin and A. A. Yushkebich

10) Cf. K. Ito and H. P. McKean [14] and G. Maruyama [18]. 
$[8], 1)$ and 2) imply the strong Markov property.

Next we shall define a new Markov process following M. Kac [16]. Attach a distingished coordinate on killing time $\sigma_{(\infty)} \in$ $[0,+\infty]$ to $w$; and extend $P_{(1, \theta)}^{(\alpha)},(1, \theta) \in \partial D$, to $\mathscr{B}^{0} \times \mathscr{F}([0,+\infty])$ so that

$$
P_{(1, \theta)}^{(\alpha)}\left(\sigma_{\{\infty)}>t / \mathscr{B}\right)=e^{-\alpha t^{-1}(t, w)}, \quad t \geq 0:
$$

let

$$
P_{(\infty)}^{(\alpha)}\left\{\theta\left(\mathrm{t}^{-1}(t, w), w\right)=\infty ; t \geq 0\right\}=P_{\left\{_{\infty}\right\}}\left\{\sigma_{\{\infty\}}=0 / \mathscr{B}\right\}=1
$$

let

$$
z\left(t, w^{*}\right)= \begin{cases}z(t, w), & t<\sigma_{\{\infty\}}, \\ \infty, & t \geq \sigma_{\{\infty\}},\end{cases}
$$

where $z(t, w)=\theta\left(\mathfrak{t}^{-1}(t, w), w\right)$.

Then we have

Proposition 4. 4. $\left\{z\left(t, w^{*}\right), t \geq 0, \mathscr{B} \times \mathscr{E}([0,+\infty]), P_{x}^{(\alpha)}, x \in\right.$ $\partial D \cup\{\infty\}\}$ is a version of strong Markov process $\mathfrak{M}^{(\alpha)}=\left\{W^{\infty}, z\left(t, w^{\alpha}\right)\right.$, $\left.\mathcal{B}^{\alpha}, P_{\theta}^{(\alpha)},(1, \theta) \in \partial D\right\}$ satisfying the following properties:

1) $T_{t}^{(\alpha)}$ is strongly continuous, and

2) $\mathcal{C}(\partial D)$ is invariant under $T_{t}^{(\alpha)}$, where $T_{t}^{(\alpha)}$ is the semi-group corresponding to $\mathfrak{M}^{(\infty)}$.

PROOF. To show that $\left\{z\left(t, w^{*}\right), t \geq 0, \mathscr{B} \times \mathscr{F}([0,+\infty]), P_{x}^{(\alpha)}\right.$, $x \in \partial D \cup\{\infty\}\}$ is Markov, it is enough to show that

$$
\begin{aligned}
& E_{(1, \theta)}^{(\alpha)}\left\{f\left(w_{s}^{*-}\right) g\left(z\left(t, w^{*}\right)\right)\right\} \\
& \quad=E_{(1, \theta)}^{(\alpha)}\left\{f\left(w_{s}^{*-}\right) E_{\left(1, z\left(s, w^{*}\right)\right.}^{(\alpha)}\left\{g\left(z\left(t-s, w^{*}\right)\right)\right\}\right\}, \quad t, s \geq 0,
\end{aligned}
$$

holds for any Borel measurable $g$ and $f$.

This equation is evident when $g$ is constant and therefore substituting $g-g(\infty)$ for $g$, we can suppose that $g(\infty)=0$; also if

$$
\beta\left(w^{*}\right)= \begin{cases}0, & \text { if } \sigma_{\{\infty\}} \leq t, \\ \beta(w), & \text { if otherwise, }\end{cases}
$$

then

$$
E_{(1, \theta)}^{(\alpha)}\left(\beta\left(w^{*}\right)\right)=E_{(1, \theta)}\left\{\beta(w), \sigma_{\infty}>t\right\}=E_{(1, \theta)}\left\{\beta(w) \exp \left[-\alpha \mathrm{t}^{-1}(t, w)\right]\right\},
$$


and, since $g(\infty)=0, \beta\left(w^{*}\right)=g\left(z\left(t, w^{*}\right)\right) f\left(w_{s}^{*-}\right)$ satisfies (4.13). Finally we have

$$
\begin{aligned}
& E_{(1, \theta)}^{(\alpha)}\left\{f\left(w_{s}^{*-}\right) g\left(z\left(t, w^{*}\right)\right)\right\}=E_{(1, \theta)}^{(\alpha)}\left\{f\left(w_{s}^{*-}\right) g(z(t, w)) \exp \left[-\alpha \mathrm{t}^{-1}(t, w)\right]\right\} \\
& =E_{(1, \theta)}\left\{f\left(\theta\left(\mathrm{t}^{-1}\left(\cdot, w_{\mathfrak{t}^{-1}(s, w)}^{-}\right), w_{\mathfrak{t}^{-}{ }^{-1}(s, w)}\right)\right) e^{-\alpha \mathrm{t}^{-1}(s, w)} \times\right. \\
& \text { (4. 14) } \left.\left.\times g\left(\theta\left(\mathrm{t}^{-1}\left(t-s, w_{\mathrm{t}^{-1}(s, w)}^{+}\right)\right), w_{\mathrm{t}^{-1}(s, w)}^{+}\right)\right) \exp \left[-\alpha \mathrm{t}^{-1}\left(t-s, w_{\mathrm{t}^{-1}(s, w)}^{+}\right)\right]\right\} \\
& =E_{(1, \theta)}\left\{f\left(\theta\left(\mathrm{t}^{-1}\left(\cdot, w_{\mathrm{t}^{-1}(s, w)}^{-1}\right), w_{\mathrm{t}^{-1}(s, w)}^{-1}\right)\right) \exp \left[-\alpha \mathrm{t}^{-1}(s, w)\right] \times\right. \\
& \left.\times E_{\left(1, \theta\left(\mathrm{t}^{-1}(s, w), w\right)\right)}\left\{g\left(\theta\left(\mathrm{t}^{-1}(t-s, w), w\right)\right) \exp \left[-\alpha \mathrm{t}^{-1}(t-s, w)\right]\right\}\right\} \\
& =E_{(1, \theta)}\left\{f\left(\theta\left(\mathfrak{t}^{-1}\left(\cdot, w_{\mathrm{t}^{-1}(s, w)}^{-1}\right), w_{\mathrm{t}^{-1}(s, w)}^{-}\right)\right) \exp \left[-\alpha \mathrm{t}^{-1}(s, w)\right] \times\right. \\
& \times E_{\left(1, \theta\left(\mathrm{t}^{-1}(s, w), w\right)\right)}^{(\alpha)}\left\{\left(g\left(z\left(t-s, w^{*}\right)\right)\right\}\right\} .
\end{aligned}
$$

Bút $\beta\left(w^{*}\right)=f\left(w_{s}^{*-}\right) E_{\left(1, z\left(s, w^{*}\right)\right)}^{(\alpha)}\left(g\left(z\left(t-s, w^{*}\right)\right)\right)$ also satisfies (4.13), thus (4.14) implies (4.12).

In the similar way to that in the proof of Proposition 4.3 we can prove 1) and 2).

To avoid repeations we introduce

Definition. A strong Markov process $\mathfrak{M}^{(\alpha)}$ will be called the boundary process of order $\alpha \geq 0$. In particular $\mathfrak{M}^{(0)}$ is called simply the boundary process.

In the sequel we may consistently use the following

Notations. $\quad T_{t}^{(\alpha)}, t \geq 0$, and $K_{\gamma}^{(\alpha)}, \gamma \geq 0$, denote the semi-group and the Green operator corresponding to the boundary process of order $\alpha$ respectively, that is

$$
T_{t}^{(\alpha)} f(\theta)=E_{\theta}^{(\alpha)}\left\{f\left(z\left(t, w^{(\alpha)}\right)\right)\right\}, \quad t \geq 0,
$$

and

$$
\text { (4.16) } K_{\gamma}^{(\alpha)} f(\theta)=\int_{0}^{+\infty} e^{-\gamma t} T_{t}^{(\alpha)} f(\theta) d t\left(\equiv E_{\theta}^{(\alpha)}\left\{\int_{0}^{+\infty} e^{-\gamma t} f\left(z\left(t, w^{((\gamma)}\right)\right) d t\right\}\right) \text {. }
$$

Denoting the infinitiesimal generator of the semi-group $T_{t}^{(\alpha)}$ in Hille-Yofida's sense by $\mathfrak{H}^{(\alpha)}$ and its domain by $\mathscr{D}\left(\mathfrak{P}^{(\alpha)}\right)$.

We are now going to prove the following theorem which gives us the relation between $\mathfrak{M}$ and $\mathfrak{M g}^{(\alpha)}$ which will be useful to prove that our diffusion satisfies the boundary condition $\left.(0.4)\left(\delta_{(}^{\prime} \theta\right) \equiv 0\right)$. 
THEOREM 4.1.1. ${ }^{11}$. 1) A function $u=G_{\infty} f$ in the common range $\mathscr{R}$ of $G_{\alpha}, \alpha>0$, can be expressed in the form

$$
u(1, \theta)=K_{0+}^{(\alpha)}\left(\frac{\partial}{\partial n} \widetilde{G}_{\alpha}^{0} f\right)(\theta), \text { for any }(1, \theta) \in \partial D .
$$

2) For any $\alpha, \beta>0$

(4.18) $K_{\gamma}^{(\alpha)} g(\theta)-K_{\gamma}^{(\beta)} g(\theta)+(\alpha-\beta) G_{\beta}^{*(\gamma)}\left(h_{1}^{\alpha} K_{\gamma}^{(\alpha)} g\right)(\theta)=0, \quad g \in \mathcal{C}(\partial D)$, and

$$
\mathfrak{P}^{(\beta)} u=\mathfrak{I}^{(\alpha)} u+(\alpha-\beta) \frac{\partial}{\partial n} \tilde{G}_{x}^{n} h_{1}^{\alpha} u(\theta)
$$

where

$$
G_{\beta}^{*(\gamma)} g(\theta)=E_{(1, \theta)}\left\{\int_{0}^{+\infty} e^{-\gamma s} e^{-\gamma t\left(t, w^{\prime \prime}\right)} g(r(s, w), \theta(s, w)) d s\right\}, \quad g \in \mathcal{C}\left(\bar{D}_{r_{0}}\right) .
$$

We shall begin with several preliminary lemmas.

Lemma 4.3. For any $f(r, \theta) \in \mathcal{C}\left(\bar{D}_{r_{0}}\right)$,

$$
\frac{\partial}{\partial r} \widetilde{G}_{\alpha}^{n} f(r, \theta) \in \mathcal{C}\left(D_{r_{0}} \cup \partial D\right), \quad \alpha \geq 0 .
$$

Proof. If $f(r, \theta) \in \mathcal{C}\left(\bar{D}_{r_{1}}\right)$, then there exists, for any given $\varepsilon>0$, a function $\tilde{f}(r, \theta)$ such that

$$
\tilde{f}(r, \theta) \in C^{3}\left(\bar{D}_{r_{0}}\right) \quad \text { and } \quad|f(r, \theta)-\tilde{f}(r, \theta)|<\varepsilon .
$$

Then

$$
\left|\widetilde{G}_{x}^{0} f(r, \theta)-\widetilde{G}_{x}^{0} \tilde{f}(r, \theta)\right| \leq \varepsilon p(r),
$$

where

$$
p(r)=E_{r}^{(1)}\left\{\int_{0}^{\sigma\left(r_{0}, 1\right)(w(1))} e^{-\alpha t} d t\right\}+\frac{1}{\alpha} E_{r}^{(1)}\left\{e^{-\alpha \sigma_{r_{0}}(w(1))} ; \sigma_{r_{0}}\left(w^{(1)}\right)<\sigma_{1}\left(w^{(1)}\right)\right\} .
$$

On the other hand using Lemmas 4.1 and 4.2 we have

$$
\tilde{G}_{a}^{0} \tilde{f}(r, \theta) \in \mathcal{C}^{2}\left(\bar{D}_{r_{0}}\right),
$$

and by the definition, the left derivative $\frac{d^{-}}{d r} p(1)$ of $p(r)$ at 1 exists and is finite.

11) See. T. Ueno [20] and [21]. 
Therefore there exists $\frac{\partial}{\partial n} \tilde{G}_{a}^{0} f(1, \theta)$ and $\frac{\partial}{\partial n} \tilde{G}_{a}^{0} f(1, \theta) \in \mathcal{C}(\partial D)$. Since $\frac{\partial}{\partial r} G_{a}^{0} f(r, \theta) \in \mathcal{C}\left(D_{r_{0}}\right)$ and $\frac{\partial}{\partial r} h_{2}^{\alpha} f(r, \theta) \in \mathcal{C}\left(D_{r_{0}} \cup \partial D\right)$, we can prove in the usual way that $\frac{\partial}{\partial r} \widetilde{G}_{a}^{0} f(r, \theta) \in \mathcal{C}\left(D_{r_{0}} \cup \partial D\right)$.

LEMMA 4. 4. For any integer $n \geq 1$,

$$
E_{1}^{(1)}\left\{\left(\sigma_{1-\varepsilon}\left(w^{(1)}\right)\right)^{n}\right\}=O\left(\varepsilon^{2 n}\right), \quad \text { as } \varepsilon \downarrow 0,
$$

and

$$
E_{1}^{(1)}\left\{\left(\mathrm{t}\left(\sigma_{1-\varepsilon}\left(w^{(1)}\right), w^{(1)}\right)\right)^{n}\right\}=O\left(\varepsilon^{n}\right), \quad \text { as } \varepsilon \downarrow 0 .
$$

PROOF. It is easily seen that

$$
\begin{aligned}
E_{1}^{(1)}\left\{\left(\sigma_{1-\varepsilon}(w)\right)^{n}\right\}=n ! \int_{1-\varepsilon}^{1} & \cdots \\
& \int_{1-\varepsilon}^{1} g_{\varepsilon}\left(1, r_{1}\right) g_{\varepsilon}\left(r_{1}, r_{2}\right) \cdots \\
& g_{\varepsilon}\left(r_{n-1}, r_{n}\right) d m\left(r_{1}\right) d m\left(r_{2}\right) \cdots d m\left(r_{n}\right),
\end{aligned}
$$

where

$$
g_{\varepsilon}\left(r, r^{\prime}\right)= \begin{cases}s\left(r^{\prime}\right)-s(1-\varepsilon), & \text { for } r^{\prime}<r, \\ s(r)-s(r-\varepsilon), & \text { for } r^{\prime} \geq r\end{cases}
$$

Hence

$$
E_{0}^{(1)}\left\{\left(\sigma_{1-\varepsilon}\left(w^{(1)}\right)\right)^{n}\right\} \leq n !\left(\int_{1-\varepsilon}^{1} g_{\varepsilon}(1, r) d m(r)\right)^{n}=O\left(\varepsilon^{2 n}\right) .
$$

In the similar way we have

$$
E_{1}^{(1)}\left\{\left(t\left(\sigma_{1-\varepsilon}\left(w^{(1)}\right), w^{(1)}\right)\right)^{n}\right\} \leq n !\left(\int_{1-\varepsilon}^{1} g_{\varepsilon}(1, r) d \hat{m}(r)\right)^{n}=O\left(\varepsilon^{n}\right),
$$

where $\hat{m}(d r)=m(d r)+\delta_{\{1\}}(d r)$. q. e. d.

LEMMA 4.5. For any $t, 0 \leq t \leq \sigma_{r_{0}}\left(w^{(1)}\right)$, (4. 22) $\widetilde{E}\left\{|\eta(t,(1, \theta), \hat{\omega})-\theta|^{2}\right\} \leq K_{4}\left\{\left(t+t^{2}+\mathrm{t}\left(t, w^{(1)}\right)+\left(\mathrm{t}\left(t, w^{(1)}\right)\right)^{2}\right\}\right.$, where $K_{4}$ is independent of $t$ and $w^{(1)}$.

Using the similar methods to those in the proof of Lemma 2.1 the proof is easy and so is omitted.

Proof of Theorem 4.1. First we define two sequences of Markov times $\left\{\tau_{n}^{1}(w, \varepsilon), n=1,2, \cdots\right\} \quad$ and $\quad\left\{\tau_{n}^{2}(w, \varepsilon), n=0,1, \cdots\right\}$ respectively by 


$$
\begin{aligned}
& \tau^{1}(w, \varepsilon)= \begin{cases}\inf \{t ; r(t, w)=1-\varepsilon, t \geq 0\} & , \text { if such } t \text { exists } \\
+\infty & , \text { if otherwise, }\end{cases} \\
& \tau^{2}(w, \varepsilon)= \begin{cases}\inf \left\{t ; r\left(t, w_{\tau^{1}(w, \varepsilon)}^{+1}\right)=1, t \geq 0\right\}, & \text { if such } t \text { exists, } \\
+\infty & , \text { if otherwise, }\end{cases} \\
& \tau_{0}^{2}(w, \varepsilon)=0, \quad \tau_{1}^{1}(w, \varepsilon)=\tau^{1}(w, \varepsilon), \\
& \tau_{n}^{1}(w, \varepsilon)=\tau^{1}\left(w_{\tau_{n-1}^{2}(w, \varepsilon)}^{+}, \varepsilon\right)+\tau_{n-1}^{2}(w, \varepsilon), \quad n=1,2, \cdots . \\
& \tau_{n}^{2}(w, \varepsilon)=\tau^{2}\left(w_{\tau_{n}^{1}(w, \varepsilon)}^{+}, \varepsilon\right)+\tau_{n}^{1}(w, \varepsilon) \text {, }
\end{aligned}
$$

By the strong Markov property of $\mathfrak{M}$, we have

$$
\begin{aligned}
& G_{\infty} f(1, \theta) \\
& =\sum_{n=1}^{+\infty} E_{(1, \theta}\left\{e^{-\alpha \tau_{n-1}^{2}(w, \varepsilon)} E_{\left.\left(1, \theta ; \tau_{n-1}^{2}(w, \varepsilon), w\right)\right)}\left(\int_{0}^{\tau 1(w, \varepsilon)} e^{-\alpha t} f(r(t, w), \theta(t, w))\right) d t\right\} \\
& \quad+\sum_{n=1}^{+\infty} E_{(1, \theta)}\left\{e^{-\alpha\left(\tau_{n}^{1}(w, \varepsilon)\right.} \tilde{G}_{\alpha}^{0} f\left(1-\varepsilon, \theta\left(\tau_{n}^{1}(w, \varepsilon), w\right)\right)\right\}, \text { for any } \varepsilon>0 .
\end{aligned}
$$

Since for any $(1, \theta) \in \partial D$

$$
\begin{aligned}
& \left|E_{(1, \theta)}\left\{\int_{0}^{\tau_{1}} e^{-\alpha t} f(r(t), \theta(t)) d t\right\}\right| \leq 2\|f\| E_{1}^{(1)}\left\{\sigma_{1-\varepsilon}\right\}=0\left(\varepsilon^{2}\right), \\
& \left|\sum_{n=1}^{+\infty} E_{(1, \theta)}\left\{e^{-\alpha \tau_{n-1}^{2}} E_{\left(1, \theta\left(\tau_{n-1}^{2}\right)\right)}\left(\int_{0}^{\tau_{1}} e^{-\alpha t} f(r(t), \theta(t)) d t\right)\right\}\right| \\
& \leq o(1) E_{(1, \theta)}\left\{\sum_{n=1}^{+\infty} e^{-\alpha \tau_{n-1}^{2}} \varepsilon\right\}=o(1) .
\end{aligned}
$$

We have by Lemma 4.3

$$
\begin{aligned}
& \sum_{n=1}^{+\infty} E_{(1, \theta)}\left\{e^{-\alpha \tau_{n}^{1}} \tilde{G}_{a}^{0} f\left(1-\varepsilon, \theta\left(\tau_{n}^{1}\right)\right)\right\} \\
& =\sum_{n=1}^{+\infty} E_{(1, \theta)}\left\{e^{-\alpha \tau_{n}^{1}} \frac{\partial}{\partial n} \widetilde{G}_{\alpha}^{0} f\left(1, \theta\left(\tau_{n}^{1}\right)\right)\right\} \varepsilon+o(1) .
\end{aligned}
$$

On the other hand we obtain, for any $g(\theta) \in C(\partial D)$,

$$
\begin{aligned}
& \sum_{n=1}^{+\infty} E_{(1, \theta)}\left\{e^{-\alpha \tau_{n}^{1}} g\left(\theta\left(\tau_{n}^{1}\right)\right)\right\} \varepsilon-E_{(1, \theta)}\left\{\int_{0}^{+\infty} e^{-\alpha t} g(\theta(t)) \mathrm{t}(d t, w)\right\} \\
& =\sum_{n=1}^{+\infty} E_{(1, \theta)}\left\{e^{-\infty \tau_{n-1}^{2}} E_{\left.\left(1, \theta ; \tau_{n-1}^{2}\right)\right)}\left\{\left(e^{-\alpha \tau 1} g\left(\theta\left(\tau^{1}\right)\right) \varepsilon-\int_{0}^{\tau^{1}} e^{-\alpha t} g(\theta(t)) \mathrm{t}(d t)\right\}\right\},\right.
\end{aligned}
$$

and 


$$
\begin{aligned}
E_{(1, \theta)} & \left\{e^{-\alpha \tau^{1}}\left(g\left(\theta\left(\tau^{1}\right)\right) \varepsilon-\int_{0}^{\tau^{1}} e^{-\alpha t} g(\theta(t)) \mathrm{t}(d t)\right)\right\} \\
= & E_{(1, \theta)}\left\{e^{-\alpha \tau^{1}}\left(g\left(\theta\left(\tau^{1}\right)\right)-g(\theta)\right)\right\} \varepsilon \\
& +E_{(1, \theta)}\left\{\int_{0}^{\tau^{1}} e^{-\alpha t}(g(\theta)-g(\theta(t))) \mathrm{t}(d t)\right\} \\
& +E_{1}^{(1)}\left\{\varepsilon-\int_{0}^{\sigma_{1-\varepsilon}} e^{-\alpha t} \mathrm{t}(d t)\right\} g(\theta)+E_{1}^{(1)}\left\{e^{-\alpha \sigma_{1-\varepsilon}}-1\right\} g(\theta) \varepsilon .
\end{aligned}
$$

Since $\lim _{\varepsilon \downarrow 0}\left(E_{1}^{(1)}\left\{\int_{0}^{\sigma_{1-\varepsilon}} e^{-\alpha t} \mathrm{t}(d t)\right\} / \varepsilon\right)=1$, and $\lim _{\varepsilon \downarrow 0} E_{1}^{(1)}\left\{e^{-\alpha \sigma_{1-\varepsilon}}\right\}=1$, we can see that for any $(1, \theta) \in \partial D$,

$$
\begin{aligned}
& E_{(1, \theta)}\left\{e^{-\alpha \tau^{1}}\left(g\left(\theta\left(\tau^{1}\right)\right)-\int_{0}^{\tau^{1}} e^{-\alpha t} g(\theta(t)) \mathrm{t}(d t)\right\}\right. \\
& =E_{(1, \theta)}\left\{e^{-\alpha \tau^{1}}\left(g\left(\theta\left(\tau^{1}\right)\right)-g(\theta)\right)\right\} \varepsilon \\
& \quad+E_{(1, \theta)}\left\{\int_{0}^{\tau^{1}} e^{-\alpha t}(g(\theta)-g(\theta(t))) \mathrm{t}(d t)\right\} \\
& \quad+\delta^{(1)}(\varepsilon) \varepsilon,
\end{aligned}
$$

where $\delta^{(1)}(\varepsilon)$ tends to zero uniformly in $\theta$ as $\varepsilon \downarrow 0$.

By the defnition of $\mathfrak{M}$ we have

$$
\begin{gathered}
J_{1}(\theta ; \varepsilon) \equiv E_{(1, \theta)}\left\{e^{-\alpha \tau^{1}}\left(g\left(\theta\left(\tau^{1}\right)\right)-g(\theta)\right)\right\} \\
=E_{1}^{(1)}\left[e ^ { - \alpha \sigma _ { 1 - \varepsilon } } \widetilde { E } \left\{g\left(\eta\left(\sigma_{1-\varepsilon},(1, \theta), \hat{\omega}\right)\right)-g(\theta) ;\right.\right. \\
\left.\left.\left|\eta\left(\sigma_{1-\varepsilon},(1, \theta), \hat{\omega}\right)-\theta\right|<\varepsilon^{1 / 4}\right\}\right] \\
+E_{1}^{(1)}\left[e ^ { - \alpha \sigma _ { 1 - \varepsilon } } \widetilde { E } \left\{g\left(\eta\left(\sigma_{1-\varepsilon},(1, \theta), \hat{\omega}\right)\right)-g(\theta) ;\right.\right. \\
\left.\left.\left|\eta\left(\sigma_{1-\varepsilon},(1, \theta), \hat{\omega}\right)-\theta\right| \geq \varepsilon^{1 / 4}\right\}\right] .
\end{gathered}
$$

Let $M_{g}^{\varepsilon}=\max _{(1, \theta) \in \partial D} \max _{\theta^{\prime} \in U_{\varepsilon}(\theta)}\left|g\left(\theta^{\prime}\right)-g(\theta)\right|$, where $U_{\varepsilon}(\theta)=\left\{\theta^{\prime} ;\left|\theta-\theta^{\prime}\right|<\right.$ $\left.\varepsilon^{1 / 4}\right\}$. Since $g(\theta)$ is uniformly continuous in $\partial D$, we have

$$
\begin{aligned}
& \widetilde{E}\left\{\left|g\left(\eta\left(\sigma_{1-\varepsilon},(1, \theta), \hat{\omega}\right)\right)-g(\theta)\right| ;\left|\eta\left(\sigma_{1-\varepsilon},(1, \theta), \hat{\omega}\right)-\theta\right|<\varepsilon^{1 / 4}\right\} \\
& \quad \leq M_{g}^{\varepsilon} \rightarrow 0, \text { as } \varepsilon \rightarrow 0 .
\end{aligned}
$$

Using Tchebycheff's inequality, Lemmas 4.4 and 4.5 , we have

$$
\begin{aligned}
& E_{1}^{(1)}\left[e ^ { - \alpha \sigma _ { 1 - \varepsilon } } \widehat { E } \left\{\left|g\left(\eta\left(\sigma_{1-\varepsilon},(1, \theta), \hat{\omega}\right)\right)-g(\theta)\right| ;\right.\right. \\
& \left.\left.\qquad\left|\eta\left(\sigma_{1-\varepsilon},(1, \theta), \hat{\omega}\right)-\theta\right| \geq \varepsilon^{1 / 4}\right\}\right] \\
& \leq 2 K_{4}|| g|| E_{1}^{(1)}\left\{\sigma_{1-\varepsilon}+\left(\sigma_{1-\varepsilon}\right)^{2}+\mathrm{t}\left(\sigma_{1-\varepsilon}\right)+\left(\mathrm{t}\left(\sigma_{1-\varepsilon}\right)\right)^{2}\right\} / \varepsilon^{1 / 2} \\
& =\delta^{(2)}(\varepsilon),
\end{aligned}
$$


where $\delta^{(2)}(\varepsilon)$ tends to zero uniformly in $\theta$ as $\varepsilon \downarrow 0$.

Thus combining (4.28) with (4.29) we see that $J_{1}(\theta, \varepsilon)$ tends to zero uniformly in $\theta$ as $\varepsilon \downarrow 0$.

Now we shall estimate the second term of the right hand side of equation (4.26). By the definition of $\mathfrak{M}$, we have

$$
\begin{aligned}
& \varepsilon J_{2}(\theta ; \varepsilon) \equiv E_{(1, \theta)}\left\{\int_{0}^{\tau^{1}} e^{-\alpha t}(g(\theta)-g(\theta(t)) \mathrm{t}(d t)\}\right. \\
& =E_{1}^{(1)}\left[\int_{0}^{\sigma_{1-\varepsilon}} e^{-\alpha t} \widetilde{E}\{g(\theta)-g(\eta(t,(1, \theta), \hat{\omega}))\right. \\
& \left.\left.;|\theta-\eta(t,(1, \theta), \hat{\omega})|<\varepsilon^{1 / 4}\right\} \mathrm{t}(d t)\right] \\
& +E_{1}^{(1)}\left[\int_{0}^{\sigma_{1-\varepsilon}} e^{-\alpha t} \widetilde{E}\{g(\theta)-g(\eta(t,(1, \theta), \hat{\omega})) ;\right. \\
& \left.\left.|\theta-\eta(t,(1, \theta), \hat{\omega})| \geqslant \varepsilon^{1 / 4}\right\} \mathrm{t}(d t)\right], \quad \text { for }(1, \theta) \in \partial D .
\end{aligned}
$$

Now Lemmas 4.4 and 4.5 combined with the Tchbycheff's inequality, will imply

$$
\begin{aligned}
& \mid E_{1}^{(1)}\left[\int_{0}^{\sigma_{1-\varepsilon}} e^{-\alpha t} \widehat{E}\{g(\theta)-g(\eta(t,(1, \theta), \hat{\omega})) ;\right. \\
& \left.\left.\quad|\theta-\eta(t,(1, \theta), \hat{\omega})| \geqslant \varepsilon^{1 / 4}\right\} \mathrm{t}(d t)\right] \mid \\
& \quad \leq 2|| g|| E_{1}^{(1)}\left\{\sigma_{1-\varepsilon} \mathrm{t}\left(\sigma_{1-\varepsilon}\right)+\left(\sigma_{1-\varepsilon}\right)^{2} \mathrm{t}\left(\sigma_{1-\varepsilon}\right)+\left(\mathrm{t}\left(\sigma_{1-\varepsilon}\right)\right)^{2}+\left(\mathrm{t}\left(\sigma_{1-\varepsilon}\right)\right)^{3}\right\} \varepsilon^{-1 / 2} \\
& \quad=\delta^{(3)}(\varepsilon) \varepsilon,
\end{aligned}
$$

where $\delta^{(3)}(\varepsilon)$ tends to zero uniformly in $\theta$ as $\varepsilon \downarrow 0$. It is easily seen that

$$
\begin{aligned}
& \mid E_{1}^{(1)}\left[\int_{0}^{\sigma_{1-\varepsilon}} e^{-\alpha t} \widetilde{E}\{g(\theta)-g(\eta(t,(1, \theta), \hat{\omega})) ;\right. \\
& \left.\left.\quad|\theta-\eta(t,(1, \theta), \hat{\omega})|<\varepsilon^{1 / 4}\right\} \mathrm{t}(d t)\right] \mid \\
& \leq M_{g}^{\varepsilon} E_{1}^{(1)}\left(\mathrm{t}\left(\sigma_{1-\varepsilon}\right)\right)=\delta^{(4)}(\varepsilon) \varepsilon,
\end{aligned}
$$

where $\delta^{(4)}(\varepsilon)$ tends to zero uniformly in $\theta$ as $\varepsilon \downarrow 0$. By (4.30), (4.31) and (4.32), we can see that $J_{2}(\theta ; \varepsilon)$ tends to zero uniformly in $\theta$ as $\varepsilon \downarrow 0$.

Therefore

$$
\left|E_{(1, \theta)}\left\{e^{-\alpha \tau^{1}} g\left(\theta\left(\tau^{1}\right)\right) \varepsilon-\int_{0}^{\tau^{1}} e^{-a t} g(\theta(t)) \mathrm{t}(d t)\right\}\right| \leq \varepsilon \delta^{(5)}(\varepsilon),
$$

where $\delta^{(5)}(\varepsilon)$ tends to zero uniformly in $\theta$ as $\varepsilon \downarrow 0$. 
Combining (4.25) with (4.33) we can see that

$$
\sum_{n=1}^{+\infty} E_{(1, \theta)}\left\{e^{-\infty \tau_{n}^{1}(w, \varepsilon)} g\left(\theta\left(\tau_{n}^{1}(w, \varepsilon)\right)\right)\right\} \varepsilon, \quad \text { for }(1, \theta) \in \partial D
$$

tends to $E_{(1, \theta)}\left\{\int_{0}^{+\infty} e^{-\alpha t} g(\theta(t, w)) \mathrm{t}(d t, w)\right\}$ as $\varepsilon \downarrow 0$.

Applying this to (4.24) we have, by (4.22) and (4.23),

$$
G_{\alpha} f(1, \theta)=E_{(1, \theta)}\left\{\int_{0}^{+\infty} e^{-\alpha t(t, w)} \frac{\partial}{\partial n} \widetilde{G}_{\alpha}^{0} f\left(\theta\left(\mathrm{t}^{-1}(t, w), w\right)\right) d t\right\}, \quad(1, \theta) \in \partial D .
$$

Thus we have completed the proof of 1 ).

Now we shall prove 2) and 3). By the definition of $K_{\gamma}^{(\alpha)}$, we can see that for any $g(\theta) \in \mathcal{C}(\partial D)$,

$$
\begin{aligned}
& K_{\gamma}^{(\alpha)} g(\theta)-K_{\gamma}^{(\beta)} g(\theta) \\
& =E_{(1, \theta)}\left\{\int_{0}^{+\infty} e^{-\gamma \mathrm{t}(t, w)} e^{-\alpha t} \int_{0}^{t} e^{-(\beta-\alpha) s} d s g(\theta(t, w)) \mathrm{t}(d t, w)\right\}(\beta-\alpha) \\
& \left.=E_{(1, \theta)}\left\{\int_{0}^{+\infty} e^{-\beta s} e^{-\gamma(s, w)} d s \int_{0}^{+\infty} e^{-\alpha t} e^{-\gamma \mathrm{t}(t, w)+}\right) g\left(\theta\left(t, w w_{s}^{++}\right)\right) \mathfrak{t}\left(d t, w_{s}^{+}\right)\right\}(\beta-\alpha) \\
& =E_{(1, \theta)}\left\{\int_{0}^{+\infty} e^{-\beta s} e^{-\gamma(s, w)}\left(h_{1}^{\alpha} K_{0+}^{(\alpha)} g\right)(r(s, w), \theta(s, w)) d s\right\}(\beta-\alpha), \alpha, \beta>0 .
\end{aligned}
$$

Hence using 1) in theorem we have, for any $\alpha, \beta>0$,

$$
K_{\gamma}^{(\alpha)} g(\theta)-K_{\gamma}^{(\beta)} g(\theta)+(\alpha-\beta) G_{\beta}^{*(\gamma)}\left(h_{1}^{\alpha} K_{0+}^{(\alpha)} g\right)(\theta)=0 .
$$

By (4.34) and Lemma 4.3 we can prove that the range space of $K_{0+}^{(\alpha)}$ coincids with that of $K_{0+}^{(\beta)}$. Since $K_{\gamma}^{(\alpha)}, 0 \leq \gamma<+\infty$, are the Green operators for $\mathfrak{M}^{(\alpha)}$, the range space of $K_{\gamma}^{(\alpha)}$ is independent of $\gamma$. Therefore by Hille-Yosida's theory we can prove that $\mathscr{D}\left(\mathfrak{U}^{(\alpha)}\right)$ is independent of $\alpha, \alpha>0$.

Hence using (4.34) we have

$$
\left(\mathfrak{U}^{(\beta)}-\mathfrak{A}^{(\alpha)}\right) K_{0+}^{(\alpha)} g(\theta)=(\alpha-\beta) \frac{\partial}{\partial n} \widetilde{G}_{\beta}^{0} h_{1}^{\alpha} K_{0+}^{(\alpha)} g(\theta) .
$$

(4.34) and (4.35) imply 3) of Theorem 4.1. Finally we completed the proof of Theorem 4.1.

Feller's results on the boundary condition for the onedimensional diffusion will be interpreted in the terminology used 
above, and T. Ueno [20] and [21] has recently proved (4.17) in the our case using the analytical methods.

For the diffusion constructed in Section 2 we have corresponding to Theorem 4. 1 ,

THEOREM 4.2. 1) A function $u=G_{\alpha} f$ in the range $R$ of $G_{\alpha}$, $\alpha>0$, can be expressed in the form

$$
u(1, \theta)=K_{0+}^{(\alpha)}\left(f+\frac{\partial}{\partial n} \widetilde{G}_{\alpha}^{0} f\right)(\theta), \text { for any }(1, \theta) \in \partial D
$$

2) $\mathscr{D}\left(\mathfrak{U}^{(\alpha)}\right)$ is independent of the choice of $\alpha>0$.

3) For any $\alpha, \beta>0$,

$$
\begin{array}{r}
K_{0+}^{(\alpha)} g(\theta)-K_{0+}^{(\beta)} g(\theta)+(\alpha-\beta) K_{0+}^{(\beta)}\left(K_{0+}^{(\alpha)} g+\frac{\partial}{\partial n} \tilde{G}_{\beta}^{0}\left(h_{1}^{\alpha}\left(K_{0+}^{(\alpha)} g\right)\right)\right)(\theta)=0, \\
g \in \mathcal{C}(\partial D),
\end{array}
$$

and

$$
\mathfrak{P}^{(\beta)} u=\mathfrak{P}^{(\alpha)} u+(\alpha-\beta)\left(u+\frac{\partial}{\partial n} \widetilde{G}_{\beta}^{0} h_{1}^{\alpha} u\right) .
$$

This theorem is proved by using the same procedure as those in the proof of Theorem 4.1 and therefore the proof is omitted.

5. The infinitesimal generator of the boundary process and the boundary conditions. In this section we shall seek the boundary conditions corresponding to the diffusion process $M \mathcal{M}$ constructed in Section 2 or Section 3.

We shall first discuss the diffusion process $9 \mathfrak{M}$ constructed in Section 3 whose boundary condition will be given by the following Theorems 5.1 and 5.2.

THEOREM 5.1. Let (S) be the infinitesimal generator corresponding to $\mathfrak{M}$ and $\mathscr{D}((S)$ be its domain. Suppose that $u(r, \theta)$ belongs to $\mathscr{D}(\mathbb{S})$. Then

$$
\frac{\partial}{\partial n}\left[u-h_{1}^{\alpha} u\right](1, \theta)+\mathfrak{P}^{(\alpha)} u(1, \theta)=0, \text { for amy } \alpha>0
$$

And the equation

(5.2) $(\alpha-A) u(r, \theta)=f(r, \theta), \quad(r, \theta) \in D_{r_{0}}, f(r, \theta) \in \mathcal{C}^{1}\left(\bar{D}_{r_{0}}\right)$, 
has one and only one solution satisfying the following conditions

$$
\lim _{r \downarrow r_{0}} A u(r, \theta)=0, \quad\left(r_{0}, \theta\right) \in \partial D_{r_{0}}^{(1)},
$$

and for any given $\beta>0$,

$$
\frac{\partial}{\partial n}\left[u-h_{1}^{\beta} u\right](1, \theta)+\mathfrak{A}^{(\beta)} u(1, \theta)=0 .
$$

Proof. Since $u(r, \theta) \in \mathscr{D}(\mathbb{S})$, for any given $\alpha>0$, there exists a function $f(r, \theta) \in \mathcal{C}\left(\bar{D}_{r_{0}}\right)$ satisfying

$$
u(r, \theta)=G_{\alpha} f(r, \theta), \quad(r, \theta) \in \bar{D}_{r_{0}} .
$$

According to Theorem 4.1, $u(1, \theta) \in \mathscr{D}\left(\mathfrak{U}^{(\alpha)}\right)$. By (4.17) we have

$$
\mathfrak{H}^{(\alpha)} G_{\alpha} f(1, \theta)=-\frac{\partial}{\partial n} \tilde{G}_{\alpha}^{0} f(1, \theta), \quad(1, \theta) \in \partial D .
$$

Since $\tilde{G}_{a}^{0} f(r, \theta)=u(r, \theta)-h_{1}^{\alpha} u(r, \theta),(5.5)$ implies (5.1).

Let $u_{1}(r, \theta)$ and $u_{2}(r, \theta)$ be two any solution of (5.2) satisfying (5.3) and (5.4). Putting

$$
v(r, \theta)=u_{1}(r, \theta)-u_{2}(r, \theta),
$$

we can prove that

$$
(\alpha-A) v(r, \theta)=0,
$$

and that $v(r, \theta)$ satisfies the conditions (5.3) and (5.4).

Theoefore

$$
v(r, \theta)=h_{1}^{\alpha} v(r, \theta)+h_{2}^{\alpha} v(r, \theta) .
$$

Using (5.3) we have

that is

$$
0=\lim _{r \downarrow r_{0}}\left(h_{1}^{\alpha} v(r, \theta)+h_{2}^{\alpha} v(r, \theta)\right),
$$

Therefore

$$
0=\lim _{r_{\downarrow} r_{0}} h_{2}^{\alpha} v(r, \theta)=v\left(r_{0}, \theta\right) .
$$

$$
v(r, \theta)=h_{1}^{\alpha} v(r, \theta) .
$$

On the other hand by (4.17) and (5.4) we obtain that 


$$
v(1, \theta)=K_{0+}^{(\alpha)}\left[\frac{\partial}{\partial n}\left[v-h_{1}^{\alpha} v\right]\right](\theta) .
$$

Combing (5.6) with (5.7) we have

$$
v(1, \theta) \equiv 0, \quad(1, \theta) \in \partial D .
$$

Hence $v(r, \theta) \equiv 0,(r, \theta) \in \bar{D}_{r_{0}}$, that is $u_{1}(r, \theta) \equiv u_{2}(r, \theta)$.

Next in fact using Lemmas 4.1 and 4.2 and Theorem 4.1 we can prove that

$$
u(r, \theta)=\widetilde{G}_{\alpha}^{0} f(r, \theta)+h_{1}^{\alpha}\left(K_{0+}^{(\alpha)}\left(\frac{\partial}{\partial n} \widetilde{G}_{\alpha}^{0} f\right)\right)(r, \theta),
$$

is a solution of (5.2) satisfying (5.3) and (5.4).

Hence we have finally completed the proof of Theorem 5.1.

We are going to seek a certain representation of $\mathfrak{A}^{(\alpha)}$. If we impose some appropriate regularity conditions to the function $u(r, \theta), \mathfrak{A}^{(a)}$ can be written in a concrete form as will be soon proved in Theorem 5.2. First we prepare several lemmas

Lemma 5.1. Suppose $\tau_{\varepsilon}(w)$ is a random time such that

$$
\tau_{\varepsilon}(w)=\sigma_{1-\varepsilon}(w)+\sigma_{1}\left(w_{\sigma_{1-\varepsilon}(w)}^{+}\right) .
$$

Then we have

$$
\begin{gathered}
\mathfrak{A}^{(\alpha)} u(\theta)=\lim _{\varepsilon \downarrow 0} E_{(1, \theta)}\left\{\left(e^{-\alpha \tau_{\varepsilon}^{(w)}} u\left(\theta\left(\tau_{\varepsilon}(w), w\right)\right)-u(\theta)\right) / \varepsilon\right\}, \\
\text { for } u(\theta) \in \mathscr{D}\left(\mathfrak{\Re}^{(\alpha)}\right) .
\end{gathered}
$$

PROOF. The condition $u(\theta) \in \mathscr{D}\left(\mathfrak{H}^{(\alpha)}\right)$ implies that for any $\beta>0$ there exists function $f(\theta) \in \mathcal{C}(\partial D)$ satisfying

$$
u(\theta)=K_{\beta}^{(\alpha)} f(\theta), \quad(1, \theta) \in \partial D .
$$

Hence using the strong Markov property of $M$, we have

$$
\begin{gathered}
u(\theta)=E_{(1, \theta)}\left\{\int_{0}^{\mathrm{t}\left(\tau_{\varepsilon}^{(w), \varepsilon)}\right.} e^{-\beta t} e^{-\alpha \mathrm{t}^{-1}(t, w)} f\left(\theta\left(\mathrm{t}^{-1}(t, w), w\right), w\right)\right) d t^{\}} \\
+E_{(1, \theta)}\left\{e^{-\beta \mathrm{t}\left(\tau_{\varepsilon}^{(w), w)}\right.} e^{-\alpha \tau_{\varepsilon}^{(w)}} K_{\beta}^{(\alpha)} f\left(\theta\left(\tau_{\varepsilon}(w), w\right)\right)\right\} .
\end{gathered}
$$

Since $E_{(1, \theta)}\left\{\mathrm{t}\left(\tau_{\varepsilon}(w), w\right)\right\}<+\infty$, we have 
(5. 9)

$$
\begin{aligned}
& u(\theta)=-E_{(1, \theta)}\left\{\int_{0}^{\mathrm{t}\left(\tau_{\varepsilon}(w), w\right)} e^{-\alpha \mathrm{t}^{-1}(t, w)} \mathfrak{U}^{(\alpha)} u\left(\theta\left(\mathrm{t}^{-1}(t, w), w\right)\right) d t\right\} \\
& \quad+E_{(1, \theta)}\left\{u\left(\left(\theta\left(\tau_{\varepsilon}(w), w\right)\right) e^{-\alpha \tau_{\varepsilon}(w)}\right\}, \text { for any }(1, \theta) \in \partial D .\right.
\end{aligned}
$$

On the other hand by the definition of $\mathfrak{M}$ and $\mathfrak{M}^{(\alpha)}$ we have

$$
\begin{aligned}
& \left|E_{(1, \theta)}\left\{\int_{0}^{\mathrm{t}\left(\sigma_{1-\varepsilon)}\right.} e^{-\infty \mathrm{t}^{-1}(t)} \mathfrak{A}^{(\alpha)} u\left(\theta\left(\mathrm{t}^{-1}(t)\right)\right) d t\right\}-E_{(1, \theta)}\left\{\mathrm{t}\left(\tau_{\varepsilon}\right)\right\} \mathfrak{X}^{(\alpha)} u(\theta)\right| \\
& \leq\left\|\mathfrak{U}^{(\alpha)} u\right\|\left|E_{(1, \theta)}\left\{\int_{0}^{t\left(\sigma_{1-\varepsilon)}\right.}\left(e^{-\infty t^{-1}(t)}-1\right) d t\right\}\right| \\
& +E_{1}^{(1)}\left[\int _ { 0 } ^ { \mathrm { t } ( \sigma _ { 1 - \varepsilon ) } } \widetilde { E } \left\{\left|\mathfrak{Y}^{(\alpha)} u(\theta)-\mathfrak{U}^{(\omega)} u\left(\eta\left(\mathrm{t}^{-1}(t),(1, \theta), \omega\right)\right)\right| ;\right.\right. \\
& \left.\left.\left|\eta\left(\mathrm{t}^{-1}(t),(1, \theta), \omega\right)-\theta\right|>\varepsilon^{1 / 4}\right\} d t\right] \\
& +E_{1}^{(1)}\left[\int _ { 0 } ^ { \mathrm { t } ( \sigma _ { 1 - \varepsilon } ) } \widetilde { E } \left\{\left|\mathfrak{P}^{(\alpha)} u(\theta)-\mathfrak{P}^{(\alpha)} u\left(\eta\left(\mathrm{t}^{-1}(t),(1, \theta), \omega\right)\right)\right|\right.\right. \text {; } \\
& \left.\left.\left|\eta\left(\mathrm{t}^{-1}(t),(1, \theta), \omega\right)-\theta\right| \leq \varepsilon^{1 / 4}\right\} d t\right]
\end{aligned}
$$

$\equiv I_{3,1}(\varepsilon)+I_{3,2}(\varepsilon)+I_{3,3}(\varepsilon)$, say.

Using Lemma 4.4 and 4.5 and some properties of reflecting barrier Bessel process we have, by $\lim _{\varepsilon \downarrow 0} E_{(1, \theta)}\left\{\mathfrak{t}\left(\tau_{\varepsilon}(w), w\right)\right\} / \varepsilon=1$,

$$
\left|I_{3,1}(\varepsilon)\right|=o(\varepsilon) \text {, }
$$

$$
\left|I_{3,2}(\varepsilon)\right| \leq 2|| \mathfrak{A}^{(\alpha)} u|| E_{1}^{(1)}\left[\int _ { 0 } ^ { t ( \sigma _ { 1 - \varepsilon } ) } \tilde { P } \left\{\left|\eta\left(\mathrm{t}^{-1}(t),(1, \theta), \omega\right)-\theta\right|\right.\right.
$$

$$
\left|I_{3,3}(\varepsilon)\right| \leq M_{\mathfrak{H}^{(\alpha)} u}^{\varepsilon} E_{1}^{(1)}\left\{\mathrm{t}\left(\sigma_{1-\varepsilon}\right)\right\}=o(\varepsilon) .
$$

Using (5.10) and (5.11) we have

$$
\begin{gathered}
\text { (5.12) } \lim _{\varepsilon \downarrow 0} E_{(1, \theta)}\left\{\int_{0}^{\mathrm{t}\left(\sigma_{\left.1-\varepsilon^{(w)}, w\right)}\right.} e^{-\alpha \mathrm{t}^{-1}(t, w)} \mathfrak{Y}^{(\alpha)} u\left(\theta\left(\mathrm{t}^{-1}(t, w), w\right)\right) d t\right\} / \varepsilon \\
=\mathfrak{P}^{(\alpha)} u(\theta), \quad(1, \theta) \in \partial D .
\end{gathered}
$$

Hence using (5.12) it is easily seen that (5.9) implies (5.8).

Lemma 5.2. We define two random variable $X_{\varepsilon}(\hat{\omega})$ and $Y_{\varepsilon}(\hat{\omega})$ by

$$
X_{\varepsilon}(\hat{\omega})=
$$

(5.13) $\quad m(\theta) \mathrm{t}\left(\sigma_{1-\varepsilon}\left(w^{(1)}\right), w^{(1)}\right)+\beta(u, \theta) p\left([1,+\infty) \times\left[0, \mathrm{t}\left(\sigma_{1-\varepsilon}\left(w^{(1)}\right), w^{(1)}\right)\right), w^{(3)}\right)$

$$
+\beta(u, \theta) q\left([0,1) \times\left[0, \mathrm{t}\left(\sigma_{1-\varepsilon}\left(w^{(1)}\right), w^{(1)}\right)\right), w^{(3)}\right)
$$

$\left.+\sigma(\theta) g\left(\mathrm{t}\left(\sigma_{1-\varepsilon}\left(w^{(1)}\right), w^{(1)}\right)\right), w^{(3)}\right)$,

and $\quad Y_{\varepsilon}(\hat{\omega})=\eta\left(\sigma_{1-\varepsilon}\left(w^{(1)}\right),(1, \theta), \hat{\omega}\right)-X_{\varepsilon}(\hat{\omega})$. 
Then we have

1)

$$
\left[\hat{E}_{1}\left\{e^{i z X_{\varepsilon}(\omega)}\right\}\right]^{[1 / \varepsilon]}
$$

$$
\rightarrow \exp \left[i m(\theta) z-\sigma^{2}(\theta) z / 2+\int_{-\pi}^{\pi}\left(e^{i z \xi}-1-i z \xi\right) n(d \xi ; \theta),\right.
$$

as $\varepsilon \downarrow 0$, where $n(d \xi ; \theta)$ is the Lévy measure of i.d.l. satisfying (0.6).

2)

$$
\left|\hat{E}_{1}\left\{Y_{\varepsilon}(\hat{\omega})\right\}\right|=o(\varepsilon) \quad \text { and } \quad \hat{E}_{1}\left\{Y_{\varepsilon}^{2}(\hat{\omega})\right\}=o(\varepsilon) \text {. }
$$

ProOF. By (5.13)

$$
\hat{E}_{1}\left\{e^{i z X_{\varepsilon}(\hat{\omega})}\right\}=E_{1}^{(1)}\left\{e^{\left.-\mathfrak{t}\left(\sigma_{1-\varepsilon^{(}(1)}\right), w(1)\right) \psi(z)}\right\},
$$

where $\psi(z)=-i m(\theta) z+\sigma^{2}(\theta) z^{2} / 2+\int_{-\pi}^{\pi}\left(e^{i z \xi}-1-i z \xi\right) n(d \xi ; \theta)$.

Using Lemma 4.4, we have

$$
\left.E_{1}^{(1)}\left\{\exp \left[-\mathrm{t}\left(\sigma_{1-\varepsilon}\left(w^{(1)}\right), w^{(1)}\right), w^{(1)}\right) \psi(z)\right]\right\}=1-\psi r(z) \varepsilon+0\left(\varepsilon^{2}\right) .
$$

Hence

$$
\lim _{\varepsilon \downarrow 0}\left(\hat{E}_{1}\left\{e^{i z X_{\varepsilon}(\hat{\omega})}\right\}\right)^{[1 / \varepsilon]}=\exp (-\psi(z)) .
$$

$2)$ is proved by the same methods as those in the proofs of Lemma 2.1 and Theorem 4.1, using Lemma 4.3 and 4. 4, Assumptions (A. 2), (B. 2) and (C.2). Hence the proof of 2) is omitted.

Using Lemmas 5.1 and 5.2, we have

THEOREM 5.2. If $u(\theta) \in \mathcal{C}^{3}(\partial D)$, then we have

1) $u(\theta) \in \mathscr{D}\left(\mathfrak{H}^{(\alpha)}\right)$, for every $\alpha \geqq 0$.

2) $\mathfrak{U}^{(\alpha)} u(\theta)=\frac{\partial^{-}}{\partial r} h_{1}^{\alpha} u(1, \theta)+m(\theta) \frac{\partial}{\partial \theta} u(\theta)+\frac{1}{2} \sigma^{2}(\theta) \frac{\partial^{2}}{\partial \theta^{2}} u(\theta)$

$$
+\int_{-\pi}^{\pi}\left(u(\theta+\xi)-u(\theta)-\xi \frac{\partial}{\partial \theta} u(\theta)\right) n(d \xi ; \theta) .
$$

Proof. Using Lemma 5. 1, we have (5. 16) $\mathfrak{A}^{(\alpha)} u(\theta)=\lim _{\varepsilon \downarrow 0}\left[E_{(1, \theta)}\left\{e^{-\alpha \tau_{\varepsilon}^{(w)}} u\left(\theta\left(\tau_{\varepsilon}(w), w\right)\right)\right\}-u(\theta)\right] / \varepsilon, u \in \mathscr{D}\left(\mathfrak{T}^{\alpha}\right)$.

On the other hand we have, by the strong Markov property of $\mathfrak{M}$, 


$$
\begin{aligned}
& E_{(1, \theta)}\left\{e^{-\alpha \tau_{\varepsilon}^{(w)}} u\left(\theta\left(\tau_{\varepsilon}(w), w\right)\right)\right\} \\
& \quad=E_{(1, \theta)}\left\{e^{-\alpha \sigma_{1-\varepsilon^{(u)}}} h_{1}^{\alpha} u\left(1-\varepsilon, \theta\left(\sigma_{1-\varepsilon}(w), w\right)\right)\right\} \\
& =E_{(1, \theta)}\left\{h_{1}^{\alpha} u\left(1-\varepsilon, \theta\left(\sigma_{1-\varepsilon}(w), w\right)\right)\right\}+o(\varepsilon),
\end{aligned}
$$

since $\lim _{\varepsilon \downarrow 0}\left[E_{(1, \theta)}\left\{e^{-\alpha \sigma_{1-\varepsilon^{(w)}}}\right\}-1\right] / \varepsilon=0$.

Using Lemma 4.1 we have

$$
h_{1}^{\alpha} u(r, \theta) \in \mathcal{C}^{2}\left(\bar{D}_{r_{0}}\right), \quad u \in \mathcal{C}^{3}(\partial D) .
$$

Hence we can apply K. Ito's [12] Fundamental lemma (pp. 48-50) and Lemma 11 (pp. 40-41) to equation (5.16). Then using Lemma 5.2 it is easily seen that (5.15) follows from (5.16). By Hille-Yosida theory we obtain

$$
u(\theta) \in \mathscr{D}\left(\mathfrak{P}^{(a)}\right),
$$

since the right hand of $(5.15)$ is continuous in $\theta$.

In the sequel we assume that $\mathfrak{M}$ is the diffusion process constructed in Section 2. Corresponding to Theorem 5.1, we have

THEOREM 5.3. Let (s) be the infinitesimal generator corresponding to $\mathfrak{M}$ and $\mathscr{D}(\mathbb{S})$ be its domain. Suppose that $u(r, \theta)$ belongs to $\mathscr{D}(\mathbb{S})$.

Then

$$
\frac{\partial}{\partial n}\left[u-h_{1}^{\alpha} u\right](1, \theta)+\mathfrak{A}^{(\alpha)} u(1, \theta)=\lim _{r \uparrow 1} \operatorname{Bs} u(r, \theta), \text { for any } \alpha>0 .
$$

And the equation

$$
(\alpha-A) u(r, \theta)=f(r, \theta), \quad(r, \theta) \in D_{r_{0}}, \quad f(r, \theta) \in \mathcal{C}^{\prime}\left(\bar{D}_{r_{0}}\right)
$$

has one and only one solution satisfying the following conditions:

and

$$
\lim _{r \downarrow r_{0}} A u(r, \theta)=0, \quad\left(r_{0}, \theta\right) \in \partial D_{r_{0}}^{(1)},
$$

$\frac{\partial}{\partial n}\left[u-h_{1}^{\beta} u\right](1, \theta)+\mathfrak{U}^{(\beta)} u(1, \theta)=\lim _{r \uparrow 1} A u(r, \theta)$, for any given $\beta>0$.

The proof can be carried out by the same methods as those in the proof of Theorem 5.1. 
Corresponding to Theorem 5.2, we have

THEOREM 5.4. If $u(\theta) \in \mathcal{C}^{3}(\partial D)$, then we have

1) $u(\theta) \in \mathscr{D}\left(\mathfrak{P}^{(\alpha)}\right)$, for any $\alpha \geqq 0$.

2) $\mathfrak{U}^{(\alpha)} u(\theta)=\frac{\partial^{-}}{\partial r} h_{1}^{\alpha} u(1, \theta)+(m(\theta)+a(1, \theta)) \frac{\partial}{\partial \theta} u(\theta)$

$+\frac{1}{2}\left(\sigma^{2}(\theta)+b^{2}(1, \theta)\right) \frac{\partial^{2}}{\partial \theta^{2}} u(\theta)$

$+\int_{-\pi}^{\pi}\left(u(\theta+\xi)-u(\theta)-\xi \frac{\partial}{\partial \theta} u(\theta)\right) n(d \xi ; \theta), \quad(1, \theta) \in \partial D$.

6. The rotation invariant diffusion process. A. D. Wentzell's rotation invariant diffusion with the generator $(0.3)$ and the boundary condition (0.4) is only a special case of the diffusions constructed in Sections 2 and 3. However, we shall propose another method to construct this process, making effective use of the rotation invariant property; this method is the same as the skew product method due to K. Ito and H.P. McKean [14] except some modification which will become necessary because of the boundary conditions being involved.

Let $\left\{W^{(1)}, r\left(t, w^{(1)}\right), \mathscr{B}^{(1)}, P_{r}^{(1)}, r \in[0,1]\right\}$ be a reflecting barrier Bessel process and $\mathrm{t}\left(t, w^{(1)}\right)$ be the local time of $r\left(t, w^{(1)}\right)$ at $\{1\}$. Denote a Brownian motion on $R^{1}$ by $\left\{W^{(2)}, B\left(t, w^{(2)}\right), \mathscr{B}^{(2)}, P_{0}^{(2)}\right\}$. $\left\{W^{(3)}, \xi\left(t, w^{(3)}\right), \mathscr{B}^{(3)}, P_{0}^{(3)}\right\}$ is a Lévy process whose l.c. f. is

$$
\psi(z)=\left\{i z m-\frac{\sigma^{2}}{2} z+\int_{-\pi}^{\pi}\left(e^{i z \xi}-1-i \xi z\right) n(d \xi)\right\} .
$$

Now we can form a product probability space $\left\{\Omega, \mathscr{B}, P_{r}, r \in\right.$ $\left.\left[r_{0}, 1\right]\right\}$ in the usual way, where $\Omega=W^{(1)} \times W^{(2)} \times W^{(3)}, \mathscr{B}=\mathscr{B}^{(1)} \times$ $\mathscr{B}^{(2)} \times \mathscr{B}^{(3)}$ and $P_{r}=P_{r}^{(1)} \times P_{0}^{(2)} \times P_{0}^{(3)}$. Let $\left\{\widetilde{\Omega}^{(1)}, r\left(t, r, \widetilde{w}^{(1)}\right), \tilde{B}^{(1)}, \widetilde{P}^{(1)}\right\}$ be a version of Bessel process on $[0,+\infty)$, satisfying $\widetilde{P}^{(1)}\left\{r\left(t, r, \widetilde{w}^{(1)}\right)\right.$ $=r\}=1$. Then we can form a probability space $\{\hat{\Omega}, \hat{\mathcal{B}}, \hat{P}\}$, where $\hat{\Omega}=\Omega \times \widetilde{\Omega}^{(1)}, \hat{B}=B \times \widetilde{B}^{(1)}$ and $\hat{P}=P_{1}^{(1)} \times \widetilde{P}^{(1)}$. If we write

$$
r^{*}(t, r, \hat{\omega})= \begin{cases}r\left(t, r, \widetilde{w}^{(1)}\right), & 0 \leq t \leq \sigma\left(\widetilde{w}^{(1)}, r\right), \\ r\left(t-\sigma_{1}\left(\widetilde{w}^{(1)}, r\right), w^{(1)}\right), & \text { if otherwise, }\end{cases}
$$

where 


$$
\sigma\left(\widetilde{w}^{(1)}, r\right)= \begin{cases}\inf \left\{t ; r\left(t, r, \widetilde{w}^{(1)}\right)=1, t \geqq 0\right\}, & \text { if such } t \text { exists, } \\ +\infty & , \text { if otherwise, }\end{cases}
$$

then $\left\{r^{*}(t, r, \hat{\omega}), 0 \leq t<+\infty, \hat{P}\right\}$ is a version of reflecting barrier Bessel process. We define $\{r(t r, \hat{\omega}), 0 \leq t<+\infty\}$ by

$$
r(t, r, \hat{\omega}),=r^{*}\left(t \wedge \sigma_{r_{0}}(\omega, r), r, \hat{\omega}\right), \quad 0 \leq t<+\infty,
$$

where

$$
\sigma_{r_{0}}(\hat{\omega}, r)= \begin{cases}\inf \left\{t ; r^{*}(t, r, \hat{\omega})=r_{0}, t \geq 0\right\}, & \text { if such } t \text { exists, } \\ +\infty & , \text { if otherwise. }\end{cases}
$$

In this section we shall always treat this version, and assume that $a(r), b(r) \in C((0,1])$ and $b(r)$ is strictly positive on [0.1]. It sholuld be noted that the function $b(r)$ may be discontinuous at 0 .

We define two random variables $\mathfrak{S}^{(1)}(t, r, \omega)$ and $\mathfrak{S}^{(2)}(t, r, \omega)$ by

$$
\begin{aligned}
\mathfrak{S}^{(1)}(t, r, \hat{\omega})=\int_{0}^{t} a(r(s, r, \hat{\omega})) d s \text { and } \\
\quad \mathfrak{S}^{(2)}(t, r, \hat{\omega})=\int_{0}^{t} b^{2}(r(s, r, \hat{\omega})) d s, \quad 0 \leq t<+\infty .
\end{aligned}
$$

Now we proceed to the construction of the path function. Let

$$
\begin{aligned}
\eta^{*}(t,(r, \theta), \hat{\omega})=\theta & +B\left(\mathfrak{S}^{(2)}(t, r, \hat{\omega}), w^{(2)}\right)+\mathfrak{S}^{(1)}(t, r, \hat{\omega})+ \\
& +\xi\left(\mathrm{t}\left(\left(t-\sigma_{1}\left(\widetilde{w}^{(1)}, r\right)\right) \vee 0, w^{(1)}\right), w^{(3)}\right),
\end{aligned}
$$

and

$$
\eta(t,(r, \theta), \hat{\omega})=\eta^{*}\left(t \wedge \sigma_{r_{0}}(\hat{\omega}, r),(r, \theta), \hat{\omega}\right),
$$

where $\mathrm{t}\left(s, w^{(1)}\right)=0$, for $s \leq 0$. Then the $r(t, r, \hat{\omega})$ process together with the $\eta(t,(r, \theta), \hat{\omega})$ process will give the polar coordinate of the Markov process on $\bar{D}_{r_{0}}$.

Now we turn to the construction of the diffusion process. Let $W$ be the space of path functions taking values on $\bar{D}_{r_{0}}$, and define a system of measure $P_{(r, \theta)}(\cdot)$ by

$P_{(r, \theta)}(B)=\hat{P}\{\omega: x(\cdot,(r, \theta), \hat{\omega})(\equiv(r(\cdot, r, \hat{\omega}), \eta(\cdot,(r, s), \hat{\omega}))) \in B\}, \quad B \in \mathcal{B}$.

Let

$$
\mathfrak{M}=\left\{W, x(t, w), \mathscr{B}, P_{(r, \theta)},(r, \theta) \in \bar{D}_{r_{0}}\right\} .
$$

Define the boundary process $\mathfrak{M}^{(\alpha)}, \alpha \geq 0$, using the same pro- 
cedure as those in Section 4.

Here we shall list the propositions which are easily proved using the similar methods to those in Sections $2 \sim 5$.

(A) $\mathfrak{M}$ is a markov process satisfying the following properties :

1) the semi-group $\left\{T_{t}, t \geq 0\right\}$ is strongly continuous,

2) $T_{t} f(r, \theta)$ is continuous on $\bar{D}_{r_{0}}$, if $f$ is in $\mathcal{C}\left(\bar{D}_{r_{0}}\right)$, and

3) the semi-group $\left\{T_{t}, t \geq 0\right\}$ is invariant under the rotation.

(B) Let $\&$ be the infinitesimal generator corresponding to $\mathfrak{M}$. Then if $u(r, \theta) \in \mathcal{C}^{2}\left(D_{r_{0}}\right)$,

$$
\begin{aligned}
\text { (s) } u(r, \theta)=\frac{1}{2}\left(\frac{\partial^{2}}{\partial r^{2}}+\frac{1}{r} \frac{\partial}{\partial r}+b^{2}(r) \frac{\partial^{2}}{\partial \theta^{2}}+2 a(r) \frac{\partial}{\partial \theta}\right) u(r, \theta), & \text { for }(r, \theta) \in D_{r_{0}} .
\end{aligned}
$$

(C) $\mathfrak{M}^{(\alpha)}=\left\{W^{\alpha}, z\left(t, w^{\alpha}\right), \mathscr{B}^{x}, P_{\theta}^{(\alpha)},(1, \theta) \in \partial D\right\}, \quad \alpha \geq 0$, is a strong Markov process satisfying

1) the semi-group $T_{t}^{(\alpha)}$ corresponding to $\mathfrak{M g}^{(\alpha)}$ is strongly continuous, and

2) $\mathcal{C}(\partial D)$ is invariant under $T_{t}^{(\alpha)}$.

Furthermore $\mathfrak{M}^{(\alpha)}$ is an Lévy process on $\partial D$.

(D) Theorems $4.1,5.1$ and 5.2 remain true also in this case.

(E) If $u(\theta)$ is in $\mathcal{C}^{3}(\partial D)$, then

1) $\quad \mathfrak{A}^{(\alpha)} u(\theta)=\frac{\partial^{-}}{\partial r} h_{1}^{\alpha} u(1, \theta)+m \frac{\partial}{\partial \theta} u(\theta)+\frac{1}{2} \sigma^{2} \frac{\partial^{2}}{\partial \theta^{2}} u(\theta)$

$$
+\int_{-\pi}^{\pi}\left(u(\theta+\xi)-u(\theta)-\xi \frac{\partial}{\partial \theta} u(\theta)\right) n(d \xi), \quad(1, \theta) \in \partial D,
$$

and

2)

$$
u(\theta) \in \mathscr{D}\left(\mathfrak{Y}^{\left(\alpha^{\prime}\right)}\right)
$$

Proof. Noting that for almost all fixed $\left(w^{(1)}, \widetilde{w}^{(1)}\right),\{\eta(t+s$, $(r, \theta), \hat{\omega})-\eta(s,(r, \theta), \hat{\omega})\}$ is independent of $\left\{\eta\left(t^{\prime},(r, \theta), \hat{\omega}\right), 0 \leq t^{\prime} \leq s\right\}$ and that its distribution does not depend on the valnes of $\mathrm{t}\left(s, w^{(1)}\right)$ and $\mathfrak{S}^{(1)}(s, r, \hat{\omega})$ but on the values of $\mathrm{t}\left(t, w_{s}^{(1)+}\right), \int_{s}^{t+s} a(r(\tau, r, \hat{\omega})) d \tau$ and $\int_{s}^{t+s} b^{2}(r(\tau, r, \hat{\omega})) d \tau$, we can prove that $\mathfrak{M}$ has Markov property 
by the same arguments as those in Section 2. Next noting that if $\left(r_{n}, \theta_{n}\right) \rightarrow(r, \theta)$, there exists a subsequence $\left(r_{n_{k}}, \theta_{n_{k}}\right)$ of $\left(r_{n}, \theta_{n}\right)$ such that for almost all $\omega$

$$
x\left(t,\left(r_{n_{k}}, \theta_{n_{k}}\right), \omega\right) \rightarrow x(t,(r, \theta), \omega),
$$

and $x(t,(r, \theta), \omega)$ belongs to $d_{1}$-class, we can complete the proof of (A).

The proof of (B) is similar to the proof of Proposition 4.1. The proof of $(\mathrm{C})$ is easy and so is omitted.

The proofs of (D) and (E) are exactly the same as those of Theorems 4.1, 5.1 and 5.2. Hence we shall mention only the outline of the proof. It is easily seen that

$1^{\circ}$ ) For any $t, 0 \leq t \leq \sigma_{r_{0}}\left(w^{(1)}\right)$, there exists a constant $K_{5}$ such that for almost all $\left(P_{1}^{(1)}\right) w^{(1)}$,

$$
\begin{aligned}
E_{0}^{(2)} \times E_{0}^{(3)}\left\{\left|\eta\left(\mathfrak{t}^{-1}\left(t, w^{(1)}\right),(1, \theta), \omega\right)-\theta\right|^{2}\right\} \\
\leq K_{5}\left\{\mathfrak{t}^{-1}\left(t, w^{(1)}\right)+\left(\mathfrak{t}^{-1}\left(t, w^{(1)}\right)\right)^{2}+t\right\},
\end{aligned}
$$

where $K_{5}$ is independent of $w^{(1)}$ and $t$.

$2^{\circ}$ ) Consider a random variable such that

$$
\tau_{\varepsilon}(w)=\sigma_{1-\varepsilon}(w)+\sigma_{1}\left(w_{\sigma_{1-\varepsilon}(w)}^{+}\right) .
$$

Then

$$
\begin{array}{r}
\mathfrak{P}^{(\alpha)} u(\theta)=\lim _{\varepsilon \downarrow 0}\left[E_{(1, \theta)}\left\{e^{-\alpha \tau_{\varepsilon}(w)} u\left(\theta\left(\tau_{\varepsilon}(w), w\right)\right)\right\}-u(\theta)\right] / \varepsilon \\
\text { for } u(\theta) \in \mathscr{D}\left(\mathfrak{A}^{(\alpha)}\right) .
\end{array}
$$

$\left.3^{\circ}\right)$ Consider a random variable $Y_{\varepsilon}(\hat{\omega})$ such that

$$
Y_{\varepsilon}(\hat{\omega})=\eta\left(\sigma_{1-\varepsilon}\left(w^{(1)}\right),(1, \theta), \hat{\omega}\right)-\theta-\xi\left(\mathrm{t}\left(\sigma_{1-\varepsilon}\left(w^{(1)}\right), w^{(1)}\right), w^{(3)}\right) .
$$

Then

$$
\left|E_{1}\left(Y_{\mathrm{\varepsilon}}(\hat{\omega})\right)\right|=\circ(\varepsilon) \quad \text { and } \quad E_{1}\left\{\left(Y_{\mathrm{\varepsilon}}(\hat{\omega})\right)^{2}\right\}=\circ(\varepsilon) .
$$

$\left.4^{\circ}\right)$ Let

$$
\mathcal{P}_{\mathrm{\varepsilon}}(z)=E_{1}\left\{\exp \left[i z \xi\left(\mathrm{t}\left(\sigma_{1-\varepsilon}\left(w^{(1)}\right), w^{(1)}\right), w^{(3)}\right)\right]\right\} .
$$

Then

$$
\left(\mathcal{P}_{\mathrm{g}}(z)\right)^{[1 / \varepsilon]} \rightarrow \exp \psi(z), \quad \text { as } \varepsilon \downarrow 0
$$


Noting $\left.1^{\circ}\right) \sim 4^{\circ}$ ) and repeating the same arguments as in Theorems $4.1,5.1$ and 5.2 we can prove (D) and (E).

In the sequel we assume that $\left\{W^{(1)}, r\left(t, w^{(1)}\right), \mathscr{B}^{(1)}, P_{r}^{(1)}, r \in\right.$ $[0,1]\}$ is a sticky barrier Bessel process. Then following the same procedures as above we can also define a diffusion $\mathfrak{M}$ on $\bar{D}_{r_{0}}$ and a boundary process $\mathfrak{M}^{(\alpha)}, \alpha \geq 0$, on $\partial D$. Now the proposition $(\mathrm{A}) \sim(\mathrm{E})$ will hold if we replace Theorems 4.1, 5.1 and 5.2, and $(\mathrm{E})$ by Theorems 4.2, 5.3 and 5.4, and the following ( $\left.\mathrm{E}^{\prime}\right)$ respectively :

$\left(\mathrm{E}^{\prime}\right)$ If $u(\theta)$ is in $\mathcal{C}^{3}(\partial D)$, then

$$
\begin{aligned}
\mathfrak{X}^{(\alpha)} u(\theta) & =\frac{\partial^{-}}{\partial r} h_{1}^{\alpha} u(1, \theta)+(m+a(1)) \frac{\partial}{\partial \theta} u(\theta)+\frac{1}{2}\left(\sigma^{2}+b^{2}(1)\right) \frac{\partial^{2}}{\partial \theta^{2}} u(\theta) \\
& +\int_{-\pi}^{\pi}\left(u(\theta+\xi)-u(\theta)-\xi \frac{\partial}{\partial \theta} u(\theta)\right) n(d \xi),
\end{aligned}
$$

and $u(\theta) \in \mathscr{D}\left(\mathfrak{\Re}^{(\alpha)}\right)$.

7. Change of scale. To reduce a diffusion with the general elliptic operator (0.1) as its generator to the special case discussed above under the some rugularity assumptions we shall apply the scale and time changes ${ }^{11}$.

Under the appropriate regularity conditions we shall prove that there exists a mapping

$$
\psi: R^{2} \supset S \rightarrow D=\{(r, \theta): 0 \leq r \leq 1\},
$$

such that, in the new scale, the given elliptic differential operator $\widehat{A}^{*}$ becomes

(7.2) $\tilde{A} u(r, \theta)=m(r, \theta)\left(\frac{\partial^{2}}{\partial r^{2}}+\frac{1}{r} \frac{\partial}{\partial r}+b^{2}(r, \theta) \frac{\partial^{2}}{\partial \theta^{2}}+a(r, \theta) \frac{\partial}{\partial \theta}\right) u(r, \theta)$, $(r, \theta) \in D$,

where $b^{2}(r, \theta)$ is positive on $\bar{D}$.

Let $S$ be a bounded simply connected domain $R^{2}$, and let its boundary $\partial S$ be a twice continuously differentiable curve. Under the appropriate conditions a linear elliptic differential operator

11) See. W. Feller [9]. 


$$
\begin{aligned}
& \widetilde{A} * u(x, y)=A_{11}(x, y) \frac{\partial^{2}}{\partial x^{2}} u(x, y)+2 A_{12}(x, y) \frac{\partial^{2}}{\partial x \partial y} u(x, y) \\
& \quad+A_{22}(x, y) \frac{\partial^{2}}{\partial y^{2}} u(x, y)+A_{1}(x, y) \frac{\partial}{\partial x} u(x, y)+A_{2}(x, y) \frac{\partial}{\partial y} u(x, y)
\end{aligned}
$$

can be reduced to the simple form

$$
\begin{aligned}
A^{*} u(x, y)=M(x, y)\left\{\Delta u(x, y)+A(x, y) \frac{\partial}{\partial x} u(x, y)\right. & \\
& \left.+B(x, y) \frac{\partial}{\partial y} u(x, y)\right\} .
\end{aligned}
$$

From now on we consider only differential operator (7.4) with $M(x, y) \equiv 1$ assuming that $A(x, y)$ and $B(x, y)$ are of class $H^{1}(S)^{12)}$. Let $\partial S$ be a twice continuously differentiable curve. Let $g_{0}\left(z_{0}, z\right)$ be the Green function for the Laplace equation in the domain $S$ with singularity at $z_{0}$, and let $u(z)$ be the $\left[a_{1}, \bar{a}_{1}\right]$-pseudo-analytic function similar to $f(z)=\frac{\partial}{\partial x} g_{0}\left(z_{0}, z\right)-i \frac{\partial}{\partial y} g_{0}\left(z_{0}, z\right)$ and such that $|u / f|=1$ at $z_{0}$ and $\operatorname{Im}\{u \mid f\}=0$, where

$$
4 a(z)=-A(z)-i B(z), \quad z=(x, y) \in S .
$$

Let $z_{1}$ be some point on $\partial S$. Then the function

$$
g\left(z_{0}, z\right)=\operatorname{Re}\left\{\int_{z_{1}}^{z} u\left(z^{\prime}\right) d z^{\prime}\right\}
$$

is the Green function for the operator (7.4) with $M(x, y) \equiv 1$ in the domain $S$, with singularity at $z_{0}$. Hence there exists a constant $K_{6}$ such that

$$
\begin{aligned}
\left(\frac{\partial}{\partial x} g\left(z_{0}, z\right)\right)^{2}+ & \left(\frac{\partial}{\partial y} g\left(z_{0}, z\right)\right)^{2} \\
& \left.\geq K_{6}\left\{\left(\frac{\partial}{\partial x} g_{0}\left(z_{0}, z\right)\right)^{2}\right\}+\left(\frac{\partial}{\partial y} g_{0}\left(z_{0}, z\right)\right)^{2}\right\},
\end{aligned}
$$

and $K_{6}>0$. (See L. Bers [1], Theorem 3 and Lemma 3 or L. Beas [2]).

12) A function $u(x, y),(x, y) \in S$, will be called of class of $H^{1}(S)$, if its partial derivative of order 1 satisfy a uniform Hölder condition on every compact subset of $S$. 


\section{Therefore}

$$
\left(\frac{\partial}{\partial x} g\left(z_{0}, z\right)\right)^{2}+\left(\frac{\partial}{\partial y} g\left(z_{0}, z\right)\right)^{2}>0, \quad z \in \bar{S}-0 .
$$

Let $S_{r}$ be the neveau curve in the scalar field with velocity potential $g\left(z_{0}, z\right)$ : i. e. $S_{r}=\left\{z ; g\left(z_{0}, z\right)=r\right\}$. Then $S_{r}$ is a closed curve such that $z_{0}$ belongs to the domin enclosed by $S_{r}$, for every $r, 0 \leq r<+\infty$, and we can consider the strem function $\theta(x, y)$ satisfying $\partial \theta(z) / \partial s(z)=\partial g\left(z_{0}, z\right) / \partial n(z)$, for $z \in \partial S$, where $\partial \theta / \partial s$ means the tangential derivative of the function $\theta(z)$.

Let

$$
r(x, y)=\exp \left[-g\left(z_{0}, z\right)\right], \quad z \in \bar{S} .
$$

Then mapping $\bar{S}$ onto $D$ by

$$
\psi: \bar{S} \ni(x, y) \rightarrow(r(x, y), \theta(x, y)) \in \bar{D},
$$

and using the following equations

$$
(\partial r(z) / \partial x)(\partial \theta(z) / \partial x)+(\partial r(z) / \partial y)(\partial \theta(z) / \partial y)=0, \quad z \in \bar{S}-z_{0},
$$

and

$$
A^{*} r(z)=\left[\left(\frac{\partial}{\partial x} g\left(z_{0}, z\right)\right)^{2}+\left(\frac{\partial}{\partial y} g\left(z_{0}, z\right)\right)^{2}\right] r(z), \quad z \in S-z_{0}
$$

it is easy to see that the equation (7.4) with $M(z) \equiv 1$ can be written in the form

$$
\begin{aligned}
A^{*} u(z)=m(r(z), \theta(z))\left[\frac{\partial^{2}}{\partial r^{2}}+\frac{1}{r} \frac{\partial}{\partial r}+\hat{b}(r(z), \theta(z)) \frac{\partial^{2}}{\partial \theta^{2}}\right. \\
\left.\hat{a}(r(z), \theta(z)) \frac{\partial}{\partial \theta}\right] \tilde{u}(r(z), \theta(z)), \quad z \in S-z_{0},
\end{aligned}
$$

where $\tilde{u}(r(z), \theta(z))=u(z), m(r(z), \theta(z))=\left(r(z)\left[\left(\frac{\partial}{\partial x} g\left(z_{0}, z\right)\right)^{2}+\left(\frac{\partial}{\partial y}\right.\right.\right.$ $\left.\left.\left.g\left(z_{0}, z\right)\right)^{2}\right]\right), \hat{b}(r(z), \theta(z))=\left[\left(\frac{\partial}{\partial x} \theta(z)\right)^{2}+\left(\frac{\partial}{\partial y} \theta(z)\right)^{2}\right] / m(r(z), \theta(z))$ and $\hat{a}(r(z), \theta(z))=A^{*} \theta(z) / m(r(z), \theta(z))$, which is the equation required.

Using (7.6) we can prove that the mapping $\psi$ is a heomeomophism of $S$ onto the unit disk with center 0 .

To reduce the $A^{*}$ of $(7.8)$ to the case of $m=1$, which is the 
reduction desired, we use the random time change due to P. Lévy ${ }^{13)}$.

Example 7.1. The assumptions in this section could be make weaker for many purposes. For example, let $A^{*}$ be the Laplacian $\Delta$ and $S$ be a domain which is enclosed by the Jordan curve. Then we can take a conformal mapping satisfying $S=\psi^{-1}(D)$ as the mapping $\psi$.

\section{Part 2. The boundary value problem.}

8. The boundary value problem. Let us now consider the equation

$$
\Delta u(x)=0, \quad x \in D,
$$

with the boundary condition

$$
\frac{\partial u}{\partial n}(x)+L u(x)=-f(x), \quad x \in \partial D
$$

where $L$ is a certain linear operator acting on the subspace of $\mathcal{C}(\partial D)$. In the case $L=0$ that is well known as the second boundary value problem, while if $L$ is the multiplication by a function $A(x)$, $x \in \partial D$, we obtain the so-called third boundary value problem. The boundary value problem attached to a general linear operator $L$ was treated from the view point of the theory of differential equations by many authors such as M. I. Visik and O. A. Ladyzenskaya [23] and M. I. Visik [22].

The probabilistic approach of the boundary value problem was initiated by P. Lévy, S. Kakutani and J. L. Doob [5]. They treated only the first boundary value problem using the $N$ dimensional Brownian motion. We shall here give a probabilistic method to solve the general boundary value problem, using the Brownian motion in the unit disk associated with the general condition which is determined by $L$ in a certain manner explained later.

As is well-known, the second boundary value problem for the bounded domain is solvable only for the function $f$ satisfying

13) Cf. K. Ito and H. P. McKean [14] and G. Maruyama [18]. 


$$
\int_{\partial D} f(\theta) d \sigma(\theta)=0^{14)}
$$

which we not need such an extra condition for the first boundary value problem. From our approach of the general boundary value problem, we can see that this extra condition is necessary or not according as the Brownian motion in the unit disk refered to above is recurrent or not, although we have not yet got its rigorous proof.

Let $\left\{W^{(1)}, r\left(t, w^{(1)}\right), \mathcal{B}^{(1)}, P_{r}^{(1)}, r \in[0,1]\right\}$ be a reflecting barrier Bessel process and let $\left\{W^{(2)}, B\left(t, w^{(2)}\right), \mathcal{B}^{(2)}, P_{r}^{(2)}, r \in R^{1}\right\}$ be a Brownian motion on $R^{1}$. Denote a Lévy process whose l.c.f. is

$$
E_{0}^{(3)}\left\{e^{i z l\left(t, w^{(2)}\right)}\right\}=\exp \left\{t\left[i z m-\frac{\sigma^{2}}{2} z^{2}+\int_{-\pi}^{\pi}\left(e^{i z u}-1-i z u\right) n(d u)\right]\right\}
$$

by $\left\{W^{(3)}, l\left(t, w^{(3)}\right), \mathscr{B}^{(3)}, P_{r}^{(3)}, r \in R^{1}\right\}$. We define a version of path function $\theta(t, \theta, \omega)$ as follows. First we write

$$
\theta(t, \theta, \omega)=B\left(\mathfrak{s}\left(\mathrm{t}^{-1}\left(t, w^{(1)}\right), w^{(1)}\right), w^{(2)}\right)+l\left(t, w^{(3)}\right)+\theta,
$$

where $\mathrm{t}^{-1}\left(t, w^{(1)}\right)$ is the function inverse to the local time $\mathrm{t}\left(t, w^{(1)}\right)$ of $r\left(t, w^{(1)}\right)$ at $\{1\}$ and

$$
\mathfrak{s}\left(t, w^{(1)}\right)=\int_{0}^{t}\left\{r\left(s, w^{(1)}\right)\right\}^{-2} d s .
$$

Now can define the following operator

$$
\begin{aligned}
& K_{\beta}^{(\alpha)} g(\theta)=E_{0}^{(1)} \times E_{0}^{(2)} \times E_{0}^{(3)}\left\{\int_{0}^{+\infty} e^{-\beta t} e^{-\alpha t^{-1}\left(t, w^{(1)}\right)} g(\theta(t, \theta, \omega)) d t\right\}, \\
& \alpha, \beta>0,
\end{aligned}
$$

where $g(\theta) \in C(\partial D)$.

It is well known that for any $\alpha \geq 0$, the equation

$$
(\alpha-1 / 2 \Delta) u(r, \theta)=0 \text {, on } D,
$$

with the boundary condition

$$
u(1, \theta)=f(\theta), \quad \text { on } \quad \partial D, \quad f(\theta) \in \mathcal{C}(\partial D),
$$

has a unique solution $u(r, \theta) \in \mathcal{C}(\bar{D})$, which is represented by

14) $d \sigma(\theta)$ means the usual line element on $\partial D$. 


$$
u(x)=h^{\alpha} f(x)=\int_{\partial D} h^{\alpha}\left(x, d x^{\prime}\right) f\left(x^{\prime}\right), \quad x \in \bar{D},
$$

and the equation

$$
(\alpha-1 / 2 \Delta) u(r, \theta)=f(r, \theta), \quad(r, \theta) \in D, \quad f \in \mathcal{C}^{1}(\bar{D}),
$$

with the boundary condition

$$
u(1, \theta)=0, \quad(1, \theta) \in \partial D
$$

has a unique solution $u(x) \in C(\bar{D})$, which is represented by

$$
u(x)=G_{\alpha}^{0} f(x)=\int_{\partial D} g^{0}\left(\alpha ; x, x^{\prime}\right) f\left(x^{\prime}\right) d x^{\prime} .
$$

Let $\mathfrak{M}=\left\{W, x(t, w), \mathscr{B}, P_{x}, x \in \bar{D}\right\}$ be a diffusion process corresponding to the Green operator satisfying the following equation

$$
G_{\alpha} f(r, \theta)=G_{\alpha}^{0} f(r, \theta)+h^{\alpha}\left[K_{0+}^{(\alpha)}\left(f+\frac{\partial}{\partial n} G_{\alpha}^{0} f\right)\right](r, \theta), \quad(r, \theta) \in \bar{D} .
$$

Using the same methods as in Part 1 we can prove that such a diffusion process exists and $K_{\gamma}^{(\alpha)} g(\theta)$ satisfies the following relation

$$
K_{\gamma}^{(\alpha)} g(\theta)-K_{\gamma}^{(\beta)} g(\theta)+(\alpha-\beta) G_{\beta}^{*(\gamma)}\left\{h^{\alpha} K_{\gamma}^{(\alpha)} g\right\}(\theta)=0, \quad(1, \theta) \in \partial D .
$$

where

$$
G_{\beta}^{*(\gamma)} g(\theta)=E_{(1, \theta)}\left\{\int_{0}^{+\infty} e^{-\beta s} e^{-\gamma t(t, w)} g(r(s, w), \theta(s, w)) d s\right\}, \quad g(\theta) \in C(\bar{D}),
$$

and $\mathrm{t}(t, w)$ is the sojorn time at $\{1\}$ of $r(t, w)$.

In the same way as in Part 1 it is easily seen that $\{\theta(t, \theta, \omega)$, $\left.0 \leq t<+\infty, P_{0}^{(1)} \times P_{0}^{(2)} \times P_{0}^{(3)}\right\}$ is a version of the boundary process $\mathfrak{M}^{(0)}$ concerning the diffusion process $\mathfrak{M}$. Let $\mathfrak{A}$ be the infinitesimal generator of $\mathfrak{M}^{(0)}$. Then we have

THEOREM 8.1. 1) Let $f(\theta)$ be a continuous function defined an $\partial D$ satisfying

$$
\int_{\partial D} f(\theta) d \sigma(\theta)=0
$$

Then the equation

$$
\Delta u(r, \theta)=0, \quad(r, \theta) \in \partial D,
$$

with the boundary condition 


$$
\mathfrak{A} u(1, \theta)=-f(\theta), \quad(1, \theta) \in \partial D,
$$

has a unique (up to an additive constant) solution $u(r, \theta) \in \mathcal{C}(\bar{D})$, which is given by

$$
u(r, \theta)=G_{0+} \tilde{f}(r, \theta), \quad(r, \theta) \in \bar{D},
$$

where $\tilde{f}(r, \theta)$ is the extension of $f(\theta)$ onto the closed unit disk obtained by putting $\tilde{f}(r, \theta)=0$ inside the disk.

2) Conversely if the equation (8.6) has a solution (up to additive constant) satisfying the boundary condition (8.7), then the function $f(\theta)$ satisfies the condition (8.5).

Before proving this theorem, we shall prepare three lemmas.

LEMMA 8.1. The transition probability $P(t, x, E), x \in \bar{D}$ and $E \in \mathscr{F}(\bar{D})$ corresponding to $\mathfrak{M}$ satisfies the Döeblin condition.

Proof. Let $\Gamma$ be the circle:

$$
\left\{(r, \theta) ; r=\frac{1}{2}\right\}
$$

and let $C$ be the disk :

$$
\{(r, \theta) ; 0 \leq r \leq 1 / 4\} \text {. }
$$

It is easily seen that for every $E \in \mathscr{F}(\bar{D})$,

$$
P_{x}(x(t, w) \in E) \leq 1-P_{x}\left(x(t, w) \in \bar{D} \cap E^{c}, \sigma_{\Gamma}(w)<t\right), \quad x \in D .
$$

On ther hand we can prove that for any $\eta>0$,

$$
\begin{aligned}
& P_{x}\left(x(t, w) \in \bar{D} \cap E^{C}, \sigma_{\Gamma}(w)<t\right) \\
& \quad \geq E_{x}\left\{\sigma_{\Gamma}(w)<t ; P_{x\left(\sigma_{\Gamma}(w), w\right)}\left(x\left(t-\sigma_{\Gamma}(w), w\right) \in C \cap E^{C}\right)\right\} \\
& \quad \geq E_{x}\left\{\sigma_{\Gamma}(w)+\eta<t ; \int_{C \cap_{F}{ }^{C}} p^{0}\left(t-\sigma_{\Gamma}(w), x\left(\sigma_{\Gamma}(w), w\right), y\right) d y\right\},
\end{aligned}
$$

where $p^{0}(t, x, y) d y$ is the transition probability measure of the absorbing Brownian motion. Since $p^{0}(t, x, y)$ is continuous on $[\eta, t] \times \Gamma \times C,(8.10)$ implies that there exists a positive constant $K(t, \eta, \Gamma, C)$ satisfying

$$
\begin{aligned}
& E_{x}\left\{\sigma_{\Gamma}(w)+\eta<t ; \int_{C \cap_{E} C} p^{0}\left(t-\sigma_{\Gamma}(w), x\left(\sigma_{\Gamma}(w), w\right), y\right) d y\right\} \\
& \quad \geq K(t, \eta, \Gamma, C) P_{x}\left(\sigma_{\Gamma}(w)+\eta<t\right) \int_{C_{\cap_{E} C}} r d r d \theta
\end{aligned}
$$


By (8.9), (8.10) and (8.11) we have

$$
\begin{array}{r}
P_{x}(x(t, w) \in E) \leq 1-K(t, \eta, \Gamma, C) P_{x}\left(\sigma_{\Gamma}(w)+\eta<t\right) \int_{C \cap_{H^{C}}} r d r d \theta, \\
x \in \bar{D} .
\end{array}
$$

Now we define a finite measure $\mathcal{P}(d r d \theta)$ over $\bar{D}$ by

$$
\mathcal{P}(d r d \theta)=r d r d \theta+d \theta \delta_{\{1\}}(d r) .
$$

By the definition of $\mathfrak{M}$ we have

$P_{x}\left(\sigma_{\Gamma}(w)+\eta<t\right)=P_{r}^{(1)}\left\{\sigma_{1 / 2}\left(w^{(1)}\right)+\mathrm{t}\left(\sigma_{1 / 2}\left(w^{(1)}\right), w^{(1)}\right)+\eta<t\right\}, \quad x=\mid r, \theta(\in \bar{D}$.

Hence for fixed $\eta>0$, we can choose $t_{0}$ such that

$$
P_{x}\left(\sigma_{\Gamma}(w)+\eta<t_{0}\right) \geq 1 / 2 .
$$

Combining (8.12) with (8.13) we have

$$
P_{x}\left(x\left(t_{0}, w\right) \in E\right) \leq 1-K\left(t_{0}, \eta, \Gamma, C\right) \mathcal{P}\left(C \cap E^{C}\right) / 2, \quad x \in \bar{D} .
$$

Here we take a positive constant

$$
\varepsilon=\pi K\left(t_{0}, \eta, \Gamma, C\right) /\left(2+K\left(t_{0}, \eta, \Gamma, C\right)\right) 16 .
$$

Then we can prove that if $\varphi(E)<\varepsilon$,

$$
P_{x}\left(x\left(t_{0}, w\right) \in E\right) \leq 1-\varepsilon, \quad x \in \bar{D} .
$$

Consequently we obtain that there is a (finite-valued) measure $\varphi(\cdot)$ defined on $\mathscr{F}(\bar{D})$ with $\varphi(\bar{D})>0$, a positive $t_{0}>0$ and a positive $\varepsilon$, such that

$$
P\left(t_{0}, x, E\right) \leq 1-\varepsilon, \quad \text { if } \mathcal{P}(E) \leq \varepsilon .
$$

This $\varphi(\cdot)$ is just the measure required, (See J. L. Doob [4]).

Then we have proved Lemma 8.1.

Using Lemma 8.1 we have

LEMMA 8.2. The distribution $m(d r d \theta)$ :

$$
m(d r d \theta) \equiv\left(2 r d r+\delta_{\mathfrak{1} 1^{\prime}}(d r)\right) d \theta / 4 \pi .
$$

is stationary and for sufficicently large $T_{0}$, if $t>T_{0}$, (8.15) $|P(t, x, E)-m(E)| \leq K_{7} e^{-\delta t}$, for any $E \in \mathscr{F}(\bar{D})$ and $x \in \bar{D}$, where $K_{7}>0$ and $\delta>0$ are independent of $x$ and $E$. 
Proof. The Döeblin condition for the transition probability proved in Lemma 8.1 implies that there is exactly one stationary distribution $\tilde{m}(d r d \theta)$ satisfying (8.15). It is therefore sufficient to show that the measure $\tilde{m}(d r d \theta)$ concides with the measure $m(d r d \theta)$ defined in (8.14).

Let $\mathscr{R}^{\theta}$ be the rotation operator. Since

$$
P\left(t, \mathscr{R}^{\theta} x, \mathscr{R}^{\theta} E\right)=P(t, x, E), \quad x \in \bar{D} \text { and } E \in \mathscr{F}(\bar{D}),
$$

we obtain

$$
\widetilde{m}(E)=\tilde{m}\left(\mathcal{R}^{\theta} E\right), \quad E \in \mathscr{F}(\bar{D})
$$

which implies

$$
\tilde{m}(E)=\frac{1}{2 \pi} \iint_{E} d \theta \hat{m}(d r),
$$

and for any $f(\theta) \in \mathcal{C}([0,1])$, we get

$$
\lim _{t \uparrow+\infty} E_{(r, \theta)}\{f(r(t, w))\}=\int_{0}^{1} f(r) \hat{m}(d r) .
$$

On the other hand we can prove, by the same way as in G. Maruyama and H. Tanaka [19] (Theorem 4.3), that the one dimensional diffusion $\{r(t, w), 0 \leq t<+\infty\}$ has the stationary distribution $\frac{1}{2}\left(2 r d r+\delta_{\{1\}}(d r)\right)$. Therefore we have

$$
\hat{m}(d r)=\frac{1}{2}\left(2 r d r+\delta_{\{1\}}(d r)\right)
$$

Combining (8.17) with (8.18) we have

$$
\begin{aligned}
\tilde{m}(d r d \theta) & =\frac{1}{4 \pi}\left\{2 r d r+\delta_{(1)}(d r)\right\} d \theta \\
& =m(d r d \theta) .
\end{aligned}
$$

LEMMA 8.3. Let $g(x)$ be a bounded function defined on $\bar{D}$ satisfying

$$
\int_{\bar{D}} g(x) m(d x)=0
$$

Then $G_{\alpha} g(x)$ converges uniformly in $x$ as $\alpha \rightarrow 0$ and 


$$
G_{0+} g(x)=\int_{0}^{+\infty} T_{t} g(x) d t<+\infty, \text { for any } x \in \bar{D} .
$$

Proof. We define a measure $M(t, x, d y) d y$

$$
M(t, x, d y)=P(t, x, d y)+m(d y),
$$

and let $m(t, x, y)$ be the density function of $P(t, x, d y)-m(d y)$ with respect to $M(t, x, d y)$. Putting

$$
D_{1}=\{y ; m(t, x, y)>0\} \quad \text { and } D_{2}=\{y ; m(t, x, y) \leq 0\} \text {, }
$$

we have

$$
\begin{aligned}
\left|T_{t} g(x)-\int_{\bar{D}} g(x) m(d x)\right| \leq & || g||\left\{\left|P\left(t, x, D_{1}\right)-m\left(D_{1}\right)\right|\right. \\
& \left.+\left|P\left(t, x, D_{2}\right)-m\left(D_{2}\right)\right|\right\} .
\end{aligned}
$$

Applying Lemma 8.2 to (8.21) we obtain, for sufficiently large $T_{0}$

$$
\left|T_{t} g(x)-\int_{\bar{D}} g(y) m(d y)\right| \leq 2|| g|| K_{7} e^{-\delta t}, \quad \text { for every } t \geq T_{0} .
$$

Using the condition $\int_{\bar{D}} g(y) m(d y)=0$, we get

$\left|G_{\alpha} g(x)-\int_{0}^{+\infty} T_{t} g(x) d t\right| \leq\left\|T_{t} g\right\| \int_{0}^{T_{0}}\left|e^{-\alpha t}-1\right| d t+2 K_{7}\|g\| e^{-\delta T_{0}}, \quad x \in \bar{D}$,

which implies the uniform convergence of $G_{\alpha} g$ as $\alpha \rightarrow 0$ and (8.20).

Proof of THEOREM 8.1. Since $G_{a} \tilde{f}=h^{\alpha} K_{0+}^{(\alpha)} f, G_{a} \tilde{f}$ is continuous on $\bar{D}$. Using the condition

$$
4 \pi \int_{\bar{D}} \tilde{f}(r, \theta) m(d r d \theta)=\int_{\partial D} f(\theta) d \sigma(\theta)=0,
$$

and Lemma 8.3 we can prove that $G_{0+} \tilde{f}=\lim _{\alpha \downarrow 0} G_{\alpha} \tilde{f}$ is also continuous. Since $K_{0+}^{(\alpha)} f=G_{a \alpha} \tilde{f}$ on $\partial D, K_{0+}^{(\alpha)} f$ tends to $K_{0+}^{(0+)} f$ uniformly as $\alpha \downarrow 0$, so that $K_{0+}^{(0+)} f$ is continuous on $\partial D$.

Hence it follows from $G_{\alpha} f=h^{\alpha}\left(K_{0+}^{(\alpha)} f\right)$ that

$$
G_{0+} \tilde{f}=h^{0}\left(K_{0+}^{(0+)} f\right) .
$$

Putting $u(r, \theta)=G_{0+} \tilde{f}(r, \theta)$, (8.22) show that $u(r, \theta)$ is harmonic in $D$ and

$$
u(1, \theta)=G_{0+} \tilde{f}(1, \theta)=K_{0+}^{(0+)} f(\theta),
$$


and using the resolvent equation for $\left\{K_{\gamma}^{(0+)}\right\}$, it results from this that

$$
u(1, \theta)=K_{\gamma}^{(0+)}\left(f+\gamma K_{0+}^{(0+)} f\right)(\theta) \in \mathscr{D}(\mathfrak{U}), \quad \gamma>0,
$$

which implies

$$
\mathfrak{A} \mathfrak{l}(1, \theta)=-f(\theta), \quad(1, \theta) \in \partial D
$$

Thus the first part of our theorem was proved.

Now we shall prove the second part of Theorem 8.1. Since

$$
(\gamma-\mathfrak{A}) u(1, \theta)=\gamma u(1, \theta)-f(\theta) \in \mathcal{C}(\partial D),
$$

we get $u(1, \theta)=K_{\gamma}^{(0+)} g_{\gamma}(\theta), \gamma>0$, where $g_{\gamma}(\theta)=\gamma u(1, \theta)-f(\theta)$. By the relation (8.4) we have

$$
\left|G_{0+} \tilde{f}(1, \theta)\right|=\lim _{\alpha \downarrow 0}\left|K_{0+}^{(\alpha)} f(\theta)\right| \leq 3\|u\|, \quad(1, \theta) \in \partial D .
$$

On the other hand

$$
\begin{aligned}
& \lim _{\alpha \rightarrow 0}\left|G_{a} \tilde{f}(1, \theta)-\left[\int_{\bar{D}} \tilde{f}(\theta) d m(\theta) / \alpha\right]\right| \\
& \leqq \int_{0}^{+\infty}\left|T_{t} \tilde{f}(1, \theta)-\int_{\bar{D}} \tilde{f}(\theta) d m(\theta)\right| d t<+\infty, \quad(1, \theta) \in \partial D .
\end{aligned}
$$

Combing (8.23) with (8.24) we have

$$
\lim _{\alpha \rightarrow 0}\left[\int_{\partial D} f(\theta) d \sigma(\theta) / \alpha\right]<+\infty
$$

which implies $\int_{\partial D} f(\theta) d \sigma(\theta)=0$. This completes our proof.

The usual Neumann problem is a special case of Thorem 8.1 as is seen in the following

EXAMPLE 8.1. We assume that $l\left(t, w^{(3)}\right) \equiv 0$, that is,

$$
\theta(t, \theta, \omega) \equiv B\left(\mathfrak{s}\left(\mathrm{t}^{-1}\left(t, w^{(1)}\right), w^{(1)}\right), w^{(2)}\right)+\theta .
$$

On the other hand $\left\{\mathfrak{s}\left(\mathrm{t}^{-1}\left(t, w^{(1)}\right), w^{(1)}\right), 0 \leq t<+\infty, P_{1}^{(1)}\right\}$ is a version of stable process of order $1 / 2$ since $\left\{\log r\left(\mathfrak{g}\left(t, w^{(1)}\right), w^{(1)}\right)\right.$, $\left.0 \leq t<+\infty, P_{1}^{(1)}\right\}$ is a version of sticky barrier Brownian mition an $[0,1]$ according to K. Ito and H. P. McKean [14]. Hence using the theory of subordination we can prove that $\{\theta(t, \theta, \omega)$, $\left.0 \leq t<+\infty, P_{1}^{(1)} \times P_{0}^{(2)}\right\}$ is a version of strong Markov process with 
Poisson kernel. (Cf. S. Bochner [3] and J. L. Doob [5]).

Therefore we have

$$
E_{1}^{(1)} \times E_{0}^{(2)}\{g(\theta(t, \theta, \omega))\}=\frac{1}{2 \pi} \int_{-\pi}^{\pi} g(\xi) \frac{1-e^{-2 t}}{1+e^{-2 t}-2 e^{-t} \cos (\theta-\xi)} d \xi,
$$

and for any $g(\theta) \in \mathscr{D}(\mathfrak{R})$

$$
\mathfrak{A} g(\theta)=\lim _{\varepsilon \downarrow 0}\left[\left(h^{0} g(1-\varepsilon, \theta)-g(\theta)\right) / \varepsilon\right]=\frac{\partial}{\partial n} h^{0} g(1, \theta) .
$$

Hence applying Theorem 8.1 to this case, we can prove that for any $f(\theta) \in C(\partial D)$, the function $u(r, \theta)$ defined by

$$
u(r, \theta)=G_{0+} \tilde{f}(r, \theta)
$$

is the unique (up to additive constant) solution of the Neumann problem.

9. Remarks for the condition (8.5). The conditition

$$
\int_{\partial D} f(\theta) d \sigma(\theta)=0
$$

is indipensible for us to be able to define $u(r, \theta) \equiv G_{0+} f(r, \theta)$ in the proof of Theorem 8.1, because, if it were not for this condition, $G_{0+} f(r, \theta)$ would become indefinite by virtue of the recurrence of our process $\mathfrak{M}$.

However, in case $M \mathfrak{M}$ is non-recurrent, we can do without this condition as is seen in the following

ExAmple 9.1. In this example, let $S$ be a solid ball with radius 1 in $R^{N},(N \geq 2)$ and $G=(S)^{c}$. Let $\left\{W^{(1)}, r\left(t, w^{(1)}\right), \mathcal{B}^{(1)}, P_{r}^{(1)}\right.$, $r \in[1,+\infty)\}$ be a version of one-dimensional diffusion with Feller's invariants

$$
m(d r)=2 r^{N-1} d r, \quad \text { and } \quad s(d r)=r^{1-N} d r
$$

and the boundary condition

$$
0=\frac{d^{+}}{d r} u(1):
$$

let $\left\{W^{(2)}, B^{(N)}\left(t, w^{(2)}\right), \mathcal{B}^{(2)}, P_{a}^{(2)}, a \in \partial S\right\}$ be the Brownian motion on $\partial S$. Now we define a path function $\theta(t, \omega)$ by 
where

$$
\theta(t, \omega)=B^{(N)}\left(\mathfrak{g}\left(t, w^{(1)}\right), w^{(2)}\right), \quad 0 \leq t<+\infty
$$

and let

$$
\mathfrak{s}(t, w)=\int_{0}^{t}\left\{r\left(s, w^{(1)}\right)\right\}^{-2} d s, \quad 0 \leq t<+\infty
$$

$$
x(t, \omega)=\left(r\left(\hat{\mathfrak{t}}^{-1}\left(t, w^{(1)}\right)\right), \theta\left(\hat{\mathfrak{t}}^{-1}\left(t, w^{(1)}\right)\right)\right), \quad \hat{\mathfrak{t}}\left(t, w^{(1)}\right)=t+\mathrm{t}\left(t, w^{(1)}\right),
$$

and $\mathrm{t}\left(t, w^{(1)}\right)$ be the local time at $\{1\}$ of $r\left(t, w^{(1)}\right)$.

Then $\left\{x(t, \omega), 0 \leq t<+\infty, P_{r}^{(1)} \times P_{a}^{(2)}\right\}$ is a version of the diffusion process $M=\left\{W, x(t, w), \mathfrak{B}, P_{(r, a)}\right\}$ attached to $1 / 2 \Delta$ on $\bar{G}$.

First we treat the case $N \geq 3$. Then according to G. Maruyama and H. Tanaka [19], $\left\{W^{(1)}, r\left(t, w^{(1)}\right), \mathfrak{B}^{(1)}, P_{r}^{(1)}, r \in[1,+\infty)\right\}$ is nonrecurrent since

$$
s(+\infty)=\int_{1}^{+\infty} s(d r)=\int_{1}^{+\infty} r^{-N+1} d r<+\infty .
$$

Hence $\mathfrak{M}$ is also non-recurrent and

$$
\left|G_{a} \tilde{f}(r, a)\right| \leq\|f\| E_{r}^{(1)}\left\{\int_{0}^{+\infty} \mathrm{t}\left(d t, w^{(1)}\right)\right\}, \quad r \in[1,+\infty),
$$

where $f(a)$ is continuous function defined on $\partial S$ and

$$
\tilde{f}(r, a)= \begin{cases}f(a), & (r, a) \in \partial S, \\ 0, & \text { otherwise } .\end{cases}
$$

Hence $G_{a} \tilde{f}(r, a)$ converges to a function $G_{0+} \tilde{f}(r, a) \equiv u(r, a)$ as $\alpha$ tends to 0 . Furthermore $u(r, a)$ is continuous in $x=(r, a) \in \bar{G}$, since

$$
\left|G_{a} \tilde{f}(r, a)-G_{0+} \tilde{f}(r, a)\right| \leq\|f\| E_{r}^{(1)}\left\{\int_{0}^{1 \infty}\left(1-e^{-\alpha t}\right) \mathfrak{t}\left(d t, w^{(1)}\right)\right\} .
$$

Now we can prove in the same way as example 8.1 that $u(r, a)$ is harmonic in $G$ and satisfies the boundary condition

$$
\frac{\partial}{\partial n} u(1, a)=-f(a), \quad(1, a) \in \partial S .
$$

Hence it follows that the Neumann problem is solvable for the infinite region $G$ in $R^{N}, N \geqq 3$, for any continuous values of the normal derivative on the boundary. This fact is well known in the potential theory. 
Next we shall treat our problem in the case $N=2$. In this case the outer second boundary value problem can be reduced to the inner second boundary value problem. This fact correspond to the property that $\left[\left\{r\left(t, w^{(1)}\right)\right\}^{-1}, 0 \leq t<+\infty, P_{r}, r \in[1,+\infty)\right]$ is a version of the reflecting barrier Bessel process on [0.1]. Hence in the case $N=2$ it is easily seen that $\mathfrak{M}$ is recurrent and the Neumann problem is only solvable for the infinite region $G$ in $R^{N}$ under the condition of type (8.5).

\section{REFERENCES}

[1] L. Bers: Theory of pseudo-analytic functions. Mimeographed lecture notes. New-York Univ. (1953).

[2] L. Bers: Function theoretical properties of solutions of partial differential cquations of elliptic type. Ann. Math. Stud. No. 33 (1954), 69-94.

[3] S. Bochner: Harmonic analysis and theory of probability. Univ. Cal. Press. (1955).

[4] J. L. Doob: Stochastic processes. New York, (1953).

[5] J. L. Doob: Semi-martingales and subharmonic functions. Trans. Amer. Math. Soc. 77 (1954), 86-121.

[6] E. B. Dynkin: Infinitesimal operators of Markov processes. Theory of Prob. and Its Appl. 1 (1956), 38-59.

[7] J. L. Doob: Probability methods applied to the first boundary value problem. Proc. 3rd Berkeley Symposium on Math. Stat. and Prob. II, 49-80, Univ. Calif. Press (1956).

[8] E. B. Dynkin and A. A. Yushkebich: On strong Markov processes. Theory of Prob. and Its Appl. 1 (1956), 149-155.

[9] W. Feller: On the intrinsic form for second order differential operators. Illinois J. of Math., 2 (1958), 1-18.

[10] K. Ito: On stochastic processes (I), Japanese J. of Math. 18 (1942), 261-301.

[11] K. Ito: Stochastic differential equations in a differentiable manifold. Nagoya Math. J., 1 (1950), 35-47.

[12] K. Ito: On stochastic differential equations. Memoirs of the Amer. Math. Soc., 4 (1951).

[13] K. Ito: Stochastic processes. Tata Institute Note. to appear.

[14] K. Ito and H. P. McKean: Diffusion. to appear.

[15] S. Ito: Fundamental solutions of parabolic differential equations and boundary value problems. Japanese. J. of Math., 27 (1957), 54-102.

[16] M. Kac: On some connections between probability theory and differential and integral equations. Proc. Second Berkeley Symposium on Math. Stat. and Prob., 189-215, Univ. Calif. Press, (1951).

[17] P. Lévy: Processus stochastiques et mouvement brownien. Paris (1948).

[18] G. Maruyama: On the strong Markov property. Mem. Fac. Sci. Kyushu Univ., A, 13 (1959), 17-29.

[19] G. Maruyama and H. Tanaka: Some properties of one-dimensional diffusion processes. Mem. Fac. Sci. Kyushu Univ., A, 11 (1957), 117-141. 
[20] T. Ueno: The Brownian motion satisfying Wentzell's boundary condition. to appear in the Bulletin of the I.S.I.

[21] T. Ueno: The diffusion satisfying Wentzell's boundary condition and the Markov process on the boundary, I. Proc. Japan Acad. 36 (1960), 533-538; II, 625-629.

[22] M. I. Visik: On the stability of boundary value problems for elliptic differential equations (with respect to the variation of coefficients and right-hand sides), Dokl. Akad. Nauk SSSR, 81 (1951), 717-720.

[23] M. I. Visik and O. A. Ladyzenskaya: Boundary value problems for partial differential equations and certain classes of operator equations. Amer. Math. Soc. Translations. Ser. 2, 10, 223-281.

[24] A. D. Wentzell: On lateral conditions for multi-dimensional diffusion processes. Theory of Prob. and Its Appl., 4 (1959), 172-185. 